\title{
ULTRAFAST SUPERCONTINUUM GENERATION IN BULK CONDENSED MEDIA
}

\author{
Review \\ A. Dubietis ${ }^{\text {a }}$, G. Tamošauskas ${ }^{a}$, R. Šuminas ${ }^{\text {a }}$, V. Jukna ${ }^{\text {b, c }}$, and A. Couairon ${ }^{\text {c }}$ \\ ${ }^{a}$ Laser Research Center, Vilnius University, Sauletekio 10, LT-10223 Vilnius, Lithuania \\ ${ }^{\mathrm{b}}$ Laboratoire d'Optique Appliquée, ENSTA ParisTech, Ecole Polytechnique, Université Paris-Saclay, \\ F-91762 Palaiseau, France \\ 'Centre de Physique Théorique, CNRS, Ecole Polytechnique, Université Paris-Saclay, F-91128 Palaiseau, France \\ E-mail: audrius.dubietis@ff.vu.lt
}

Received 8 June 2017; accepted 15 June 2017

\begin{abstract}
Nonlinear propagation of intense femtosecond laser pulses in bulk transparent media leads to a specific propagation regime, termed femtosecond filamentation, which in turn produces dramatic spectral broadening, or superbroadening, termed supercontinuum generation. Femtosecond supercontinuum generation in transparent solids represents a compact, efficient and alignment-insensitive technique for generation of coherent broadband radiation at various parts of the optical spectrum, which finds numerous applications in diverse fields of modern ultrafast science. During recent years, this research field has reached a high level of maturity, both in understanding of the underlying physics and in achievement of exciting practical results. In this paper we overview the state-of-the-art femtosecond supercontinuum generation in various transparent solid-state media, ranging from wide-bandgap dielectrics to semiconductor materials and in various parts of the optical spectrum, from the ultraviolet to the mid-infrared spectral range. A particular emphasis is given to the most recent experimental developments: multioctave supercontinuum generation with pumping in the mid-infrared spectral range, spectral control, power and energy scaling of broadband radiation and the development of simple, flexible and robust pulse compression techniques, which deliver few optical cycle pulses and which could be readily implemented in a variety of modern ultrafast laser systems.
\end{abstract}

Keywords: supercontinuum generation, femtosecond filamentation, optical parametric amplification, pulse compression

PACS: 42.65.Jx, 42.65.Re

\section{Introduction}

One of the most spectacular and visually perceptible effects produced by the nonlinear propagation of intense femtosecond laser pulses in a transparent medium is dramatic broadening of the spectrum, termed white-light continuum or supercontinuum (SC) generation. At present, femtosecond SC represents a unique and versatile source of coherent radiation, whose wavelength range spans over a considerable part of the optical spectrum and by far exceeds the definition of the white light.

During the last decade or so, a remarkable progress has been achieved in femtosecond SC generation in photonic crystal [1] and microstructured [2] fibres. Technological availability of highly nonlinear materials, such as tellurite and chalcogenide glasses, fostered the development of ultrabroadband fibre-based SC sources, which deliver continuous spectral coverage from the near- to 
mid-infrared spectral ranges (see, e.g. [3, 4]). Thanks to the development of novel high peak power laser systems based on optical parametric chirped pulse amplification (OPCPA), which operate in the mid-infrared range, a series of outstanding results on high power SC generation in atmospheric air and other gases have been produced recently $[5,6$.

SC generation in bulk condensed media deserves a special place in the field due to its historical, scientific and technological importance. The first observation of the white-light continuum in a bulk solid state medium dates back to the early years of nonlinear optics, when Alfano and Shapiro discovered spectral broadening throughout the visible spectral range when focusing powerful picosecond pulses into a borosilicate glass sample [7]. The discovery was immediately followed by the observations of spectral broadening in various crystals and glasses, confirming the universal nature of the phenomenon [8], also see [9] for a complete historical account on the early developments of SC generation in various optical media.

The first femtosecond SC was generated in 1983 when Fork and co-authors reported spectral broadening from the deep-ultraviolet to the nearinfrared range by focusing intense 80 -fs pulses at $627 \mathrm{~nm}$ from the dye laser into an ethylene glycol jet [10]. This study also underlined new qualities of broadband radiation, such as improvement of pulse-to-pulse reproducibility and spatial uniformity, which benefited from the short duration of the input pulse. Since then femtosecond supercontinuum generation using dye driving lasers was demonstrated in various condensed bulk media (see [11] and references therein).

The discovery of Kerr lens mode locking, which led to the invention of a femtosecond Ti:sapphire laser oscillator [12] and was followed by the demonstration of regenerative amplification of the oscillator pulses, marked a new era in femtosecond SC generation [13]. The amplified Ti:sapphire lasers outperformed then widely spread femtosecond dye lasers in all essential parameters of operation, setting a new standard for the generation of high power femtosecond pulses [14]. The advances in femtosecond solid-state laser technology as combined with growing practical knowledge of femtosecond SC generation in transparent condensed media [15, 16] boosted the development of femtosecond optical parametric amplifiers [17], which made ultrashort broadly tunable pulses routinely available. These developments in turn facilitated the experimental studies of SC generation in the previously poorly explored mid-infrared spectral domain, provided an access to the anomalous group velocity dispersion (GVD) region of transparent media (see, e.g. [18]) and markedly extended the nomenclature of suitable nonlinear materials.

In practice, femtosecond SC generation in condensed bulk media constitutes a compact, efficient, low cost, highly robust and alignment-insensitive technique for the generation of coherent ultrabroadband radiation at various parts of the optical spectrum. As being induced by self-focusing and filamentation of intense femtosecond laser pulses, the SC radiation bears high spatial and temporal coherence [19]. The SC beam preserves the same level of spatial coherence as the input laser beam [20], or even shows its improvement due to spatial mode cleaning, which is a universal feature of the filamentation process [21], yielding an excellent focusability of the SC beam. High temporal coherence of the SC radiation stems from a well-defined temporal structure of a femtosecond filament. The SC pulses acquire a regular chirp due to material dispersion and hence exhibit good compressibility, as verified by spectral phase characterization and post-compression experiments [22]. In general, the SC radiation produced by a single filament maintains the polarization state of the input pulses; the depolarization effects start to manifest themselves only at large input powers, well exceeding the critical power for selffocusing [23-25]. The SC radiation has reasonable spectral density $(\sim 10 \mathrm{pJ} / \mathrm{nm})$ [26] and exhibits supreme statistical properties, such as mutual correlations between the intensities of spectral components, low pulse-to-pulse fluctuations, and excellent long-term stability, which compares to that of the pump laser source itself [26-31].

Altogether, these outstanding properties make femtosecond SC highly suitable for diverse applications in ultrafast spectroscopy, photonics, femtosecond technology and contemporary nonlinear optics. An extremely broad SC spectrum is on demand for transient pump-probe spectroscopy and gives an access to study ultrafast molecular 
dynamics and processes in the condensed matter with femtosecond time resolution (see, e.g. [27, 32-34). The use of SC for Z-scan measurements enables a broadband characterization of the nonlinear absorption spectra [35, 36]. Multiple filaments emerging from self-focusing of a single high power input beam exhibit mutual coherence and provide a source of multichannel white-light radiation [37, 38]. Independently generated phaselocked SC pulses [39-41] as well as parametrically amplified portions of independent SC spectra [42. 43. serve for production of frequency combs allowing for absolute high precision frequency measurements within a broad spectral range. The specific SC emission patterns, termed "ciliary white light", which emerge from the damage craters in transparent dielectric media, carry rich information on the damage profile and morphology dynamics of the ablated surface, providing a real time in situ observation of the laser ablation process [44]. Most importantly, SC is recognized as an indispensable source for seeding ultrafast optical parametric amplifiers, which provide broadly tunable femtosecond pulses [45]. The broad spectral bandwidth and compressibility of SC pulses served as an important asset that contributed to the invention of ultrabroadband noncollinear optical parametric amplifiers [46], which currently produce few optical cycle pulses at various parts of the optical spectrum, ranging from the visible to the mid-infrared spectral ranges [47]. Moreover, the SC pulses preserve the carrier envelope phase of pump pulses [48], whose stability is of key importance in determining the interaction of few optical cycle pulses with matter. The SC seeding has set new landmarks in simplifying the architecture of tabletop OPCPA technique-based laser systems [49]. Recently developed high repetition rate SC-seeded OPCPA systems provide intense few optical cycle pulses with high average power at high repetition rate [50]. SC generation in laser host crystals with sub-picosecond and picosecond laser pulses paved a new avenue in the development of compact OPCPA systems, which are built around sole picosecond oscillator/amplifier systems [51]. Finally, the SC-seeded OPCPA system delivering carrier-envelope phase-stable few-optical-cycle pulses with high average and ultrahigh peak power has been recently demonstrated [52].
In this review we present a comprehensive outlook of the results on femtosecond SC generation in transparent condensed media, which have been achieved over the last 15 years or so. The paper is organized as follows: in Section 2 we present the underlying physical picture of SC generation, which is based on femtosecond filamentation, emphasizing the temporal dynamics of SC generation and the role of material dispersion, in particular; in Section 3 we briefly discuss the governing equations and numerical models for nonlinear propagation of intense femtosecond laser pulses in transparent dielectric media; in Section 4 we provide a list of wide-bandgap dielectric materials and their optical properties, which are relevant for SC generation, and useful experimental issues that are of importance for optimizing the practical schemes of SC generation in bulk dielectric media; in Section 5 we present an overview of experimental results in commonly used wide-bandgap dielectric materials: silica and non-silica glasses, water, alkali metal fluorides, laser hosts and birefingent nonlinear crystals with second-order nonlinearity; Section 6 is devoted to SC generation in semiconductor crystals, which hold a great potential in a rapidly developing field of ultrafast mid-infrared nonlinear optics; Section 7 presents a brief summary of the experimental results aiming at SC generation with a controlled spectral extent; in Section 8 we discuss the recent developments in power scaling of the SC and pulse compression techniques based on spectral broadening; finally in Section 9 we briefly discuss other developments in the field, including generation of odd harmonics-enhanced SC, SC generation in narrow bandgap dielectric media, and SC generation with Bessel and Airy beams and optical vortices.

\section{Physical picture of supercontinuum generation}

In contrast to optical fibres, where the propagation dynamics of the optical pulse is essentially one-dimensional and the spectral broadening arises from the soliton generation and fission due to the interplay between the self-phase modulation and the material dispersion (see, e.g. [1]), the SC generation in bulk media appears to be a more complex process that involves an intricate coupling between 
spatial and temporal effects. The physical picture of SC generation in transparent bulk media could be understood in the framework of femtosecond filamentation, which provides a universal scenario of nonlinear propagation and spectral broadening of intense femtosecond laser pulses in solids, liquids and gases [53-56.

Femtosecond filamentation stems from the interplay between self-focusing, self-phase modulation, multiphoton absorption/ionization-induced free electron plasma, leading to the appearance of "a dynamic structure with an intense core, that is able to propagate over extended distances much larger than the typical diffraction length while keeping a narrow beam size without the help of any external guiding mechanism", termed femtosecond filament [54]. Generation of an extremely broadband, spatially and temporally coherent emission with a low angular divergence (or SC generation) is the most obvious manifestation of filament formation. The SC emission is accompanied by the generation of coloured conical emission, i.e. broadband radiation that is emitted at different angles with respect to the propagation axis, forming a beautiful array of concentric coloured rings. In the case of condensed media (solids and liquids) these effects are brought to a compact, few millimetre to a few centimetre length scale.

The initial stage of filament formation is a result of the intensity-dependent refractive index: $n=n_{0}+n_{2} I$, where $I$ is the intensity, $n_{0}$ is the linear refractive index. $n_{2}$ is the nonlinear refractive index related to the third-order (cubic) optical susceptibility of the material, which is positive in the transparency range of dielectric media. The induced change of the refractive index is proportional to the local intensity and thus is higher at the centre of the beam and lower at the edges. Therefore the material acts like a lens, which enforces the beam to self-focus. For a cylindrically symmetric Gaussian beam the self-focusing threshold is defined by the beam power

$$
P_{\text {cr }}=\frac{3.72 \lambda^{2}}{8 \pi n_{0} n_{2}},
$$

where $\lambda$ is the laser wavelength and which is called the critical power for self-focusing; that is the power when the effect of self-focusing precisely balances the diffractive spreading of the beam. This balance is realized for the so-called Townes beam, whose shape is close to the Gaussian beam. If the beam power exceeds $P_{\mathrm{cr}}$ the collimated input Gaussian beam will self-focus at the distance [57]

$$
z_{\mathrm{sf}}=\frac{0.367 z_{\mathrm{R}}}{\sqrt{\left[\left(P / P_{\mathrm{cr}}\right)^{1 / 2}-0.852\right]^{2}-0.0219}}
$$

which is called the nonlinear focus. Here $z_{\mathrm{R}}=\pi n_{0} w_{0}^{2} / \lambda$ denotes the Rayleigh (diffraction) length of the input Gaussian beam of the radius $w_{0}$. Although Eq. (2) is derived in the case of continuous wave laser beams, it gives a fairly accurate approximation of the nonlinear focus of femtosecond laser pulses as well. Figure 1 shows an example of the evolution of the beam radius during self-focusing of a loosely focused femtosecondpulsed Gaussian beam with power of $\sim 5 P_{\text {cr }}$ in water. The position of the nonlinear focus is indicated by the minimum beam radius.

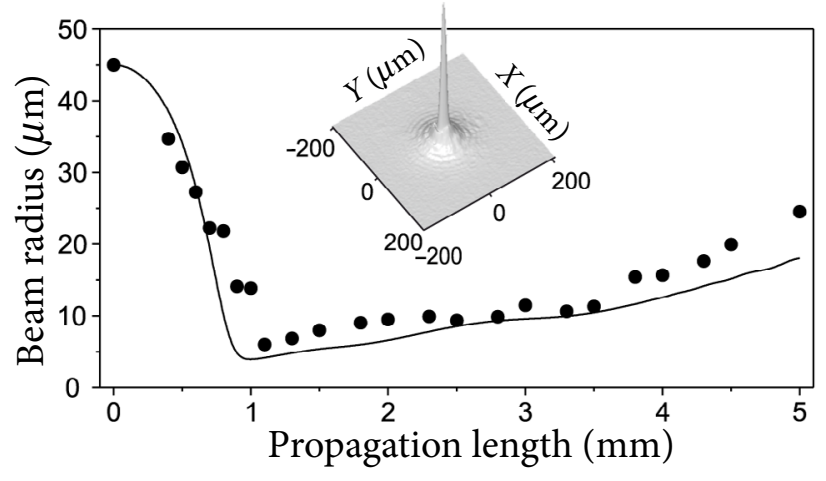

Fig. 1. FWHM beam radius as a function of propagation distance during self-focusing of a loosely focused Gaussian input beam with a $90 \mu \mathrm{m}$ FWHM diameter in water. The input beam power is $\sim 5 P_{\text {cr }}$. A solid curve shows the numerical simulation, bold dots show the experimental data. The inset shows the fluence profile of the filament, which forms after the nonlinear focus. Adapted from [58].

In the time domain, the time-varying refractive index imparts a nonlinear change in the phase of the pulse

$$
\phi_{\mathrm{nl}}(t)=-\frac{\omega_{0}}{c} n_{2} I(t) z
$$

where $\omega_{0}$ is the carrier frequency and $z$ is the propagation distance, which produces the frequency change $\delta \omega(t)=\frac{\mathrm{d}}{\mathrm{d} t} \phi_{\mathrm{nl}}(t)$ that results in the time-varying instantaneous frequency 


$$
\omega(t)=\omega_{0}+\delta \omega(t)
$$

giving rise to spectral broadening of the pulse. For the Gaussian laser pulse with the pulse duration $t_{p}$, the variation of instantaneous frequency is expressed as

$$
\delta \omega(t)=-2 \frac{\omega_{0}}{c t_{p}^{2}} n_{2} I_{0} \exp \left(-\frac{t^{2}}{t_{p}^{2}}\right) t z .
$$

The effect is called self-phase modulation and gives rise to spectral broadening by inducing a negative shift of the instantaneous frequency at the leading (ascending) front of the pulse and a positive shift of the instantaneous frequency at the trailing (descending) front of the pulse, as schematically illustrated in Fig. 2. In other words, the pulse acquires a frequency modulation corresponding to the production of red-shifted spectral components at the pulse front and blue-shifted spectral components at the pulse tail.
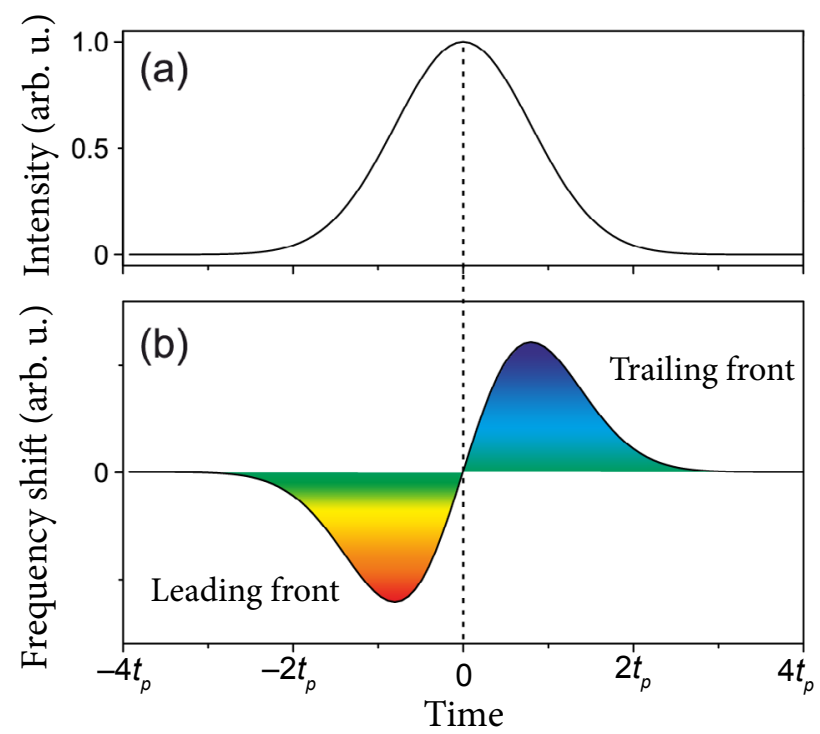

Fig. 2. Self-phase modulation of the Gaussian pulse shown in (a), which produces a variation of the instantaneous frequency shown in (b).

The self-focusing stage is a runaway effect in the sense that as the beam self-focuses, the intensity increases and so does the self-focusing effect. However, the beam cannot focus to a singularity; the beam collapse at the nonlinear focus is arrested by the multiphoton absorption and ionization, producing an energy loss and generating free electron plasma, which further absorbs and defocuses the beam. A combined action of these effects limits or clamps the intensity to a certain level. The clamping intensity depends on the order of multiphoton absorption: $K=\left\langle U_{\mathrm{g}} / \hbar \omega_{0}\right\rangle+1$, where $U$ is the bandgap, $\hbar \omega_{0}$ is the photon energy [15, 16]. The higher the order of multiphoton absorption, the higher the clamping intensity and the smaller the limiting beam diameter is at the nonlinear focus, so the larger spectral broadening is produced.

These simple considerations provide a plausible explanation of the experimentally observed bandgap dependence of the SC spectral extent and suggest that the broadest SC spectra could be attained in wide-bandgap dielectrics [15, 16. In contrast, self-focusing in the case of $K<3$ cannot produce SC. However, it is interesting to note the inverse relationship between $U_{\mathrm{g}}$ and $n_{2}$; the larger the bandgap, the smaller the value of $n_{2}$ is [59]. This is quite a paradox, since $n_{2}$ defines the strength of self-focusing and self-phase modulation, which are the fundamental physical effects behind femtosecond filamentation.

The intensity clamping effect was verified experimentally by measuring a constant width of the SC spectrum for a wide range of pulse energies above the threshold input laser energy for SC generation [60]. In condensed media the maximum clamped intensities up to tens of $\mathrm{TW} / \mathrm{cm}^{2}$ inside the nonlinear medium are estimated, accounting only for the balance between self-focusing and defocusing by free electron plasma generated by multiphoton absorption. However, plasma defocusing and absorption become relevant for the input pulse intensities of few $\mathrm{TW} / \mathrm{cm}^{2}$, contributing to significant shortening of the pulses before the nonlinear focus. If the input beam energy and focusing conditions are properly chosen, the catastrophic avalanche ionization does not come into play, so the plasma density is kept below the critical value $\left(10^{21} \mathrm{~cm}^{-3}\right)$ and optical damage of the material is avoided.

Numerical studies uncovered that besides intensity clamping, chromatic dispersion is an equally important player, which determines the extent and shape of the SC spectrum [61, 62]. The role of chromatic dispersion could be fairly evaluated in the framework of effective three wave mixing, which interprets SC generation as the emergence of new frequency components due to scattering 
of the incident optical field from material perturbation via nonlinear polarization [61, 62]. From a simple and practical viewpoint, this approach suggests that lower chromatic dispersion allows fulfilment of the phase matching condition for a broader range of scattered spectral components, that is, supports a larger spectral broadening and vice versa.

On the other hand, the interplay between the nonlinear effects and chromatic dispersion gives rise to space-time focusing and self-steepening, while the sign of GVD basically defines the emerging temporal dynamics of a femtosecond filament and so the temporal and spectral content of SC (see, e.g. [63] for an illustrative numerical study). The GVD coefficient is defined as $k_{0}^{\prime \prime}=\partial^{2} k /\left.\partial \omega^{2}\right|_{\omega_{0}}$, where $k=\omega_{0} n_{0} / c$ is the wavenumber. In the region of normal GVD $\left(k_{0}^{\prime \prime}>0\right)$, the redshifted frequencies travel faster than the blue-shifted ones, while the opposite is true in the region of anomalous GVD $\left(k_{0}^{\prime \prime}<0\right)$. Figure 3 compares the numerically simulated temporal evolution of the femtosecond filament and respective spectral dynamics in the sapphire crystal, in the ranges of normal (input wavelength $800 \mathrm{~nm})$, zero $(1.3 \mu \mathrm{m})$ and anomalous $(2.0 \mu \mathrm{m}) \mathrm{GVD}$.

\subsection{Normal GVD}

As the normal GVD of wide-bandgap dielectric materials lies in the wavelength range from the UV to the near IR that is readily accessible by modern femtosecond solid state lasers and their harmonics, it is not surprising that the SC generation in that spectral range has received the largest theoretical and experimental attention.

In the region of the normal GVD of dielectric media, pulse splitting is the effect that governs the self-focusing dynamics and the spectral broadening at and beyond the nonlinear focus. Pulse splitting in the normally dispersive dielectric medium has been foreseen theoretically using the propagation models of different complexity [64-67]. In the input power range just slightly above $P_{c r}$, pulse splitting was proposed as the mechanism which contributes in arresting the collapse of ultrashort pulses at the nonlinear focus. Theoretical predictions were afterwards confirmed experimentally by means of autocorrelation measurements [68]. A more detailed information on pulse splitting was extracted by recording the cross-correlation functions confirming the numerically predicted asymmetry between
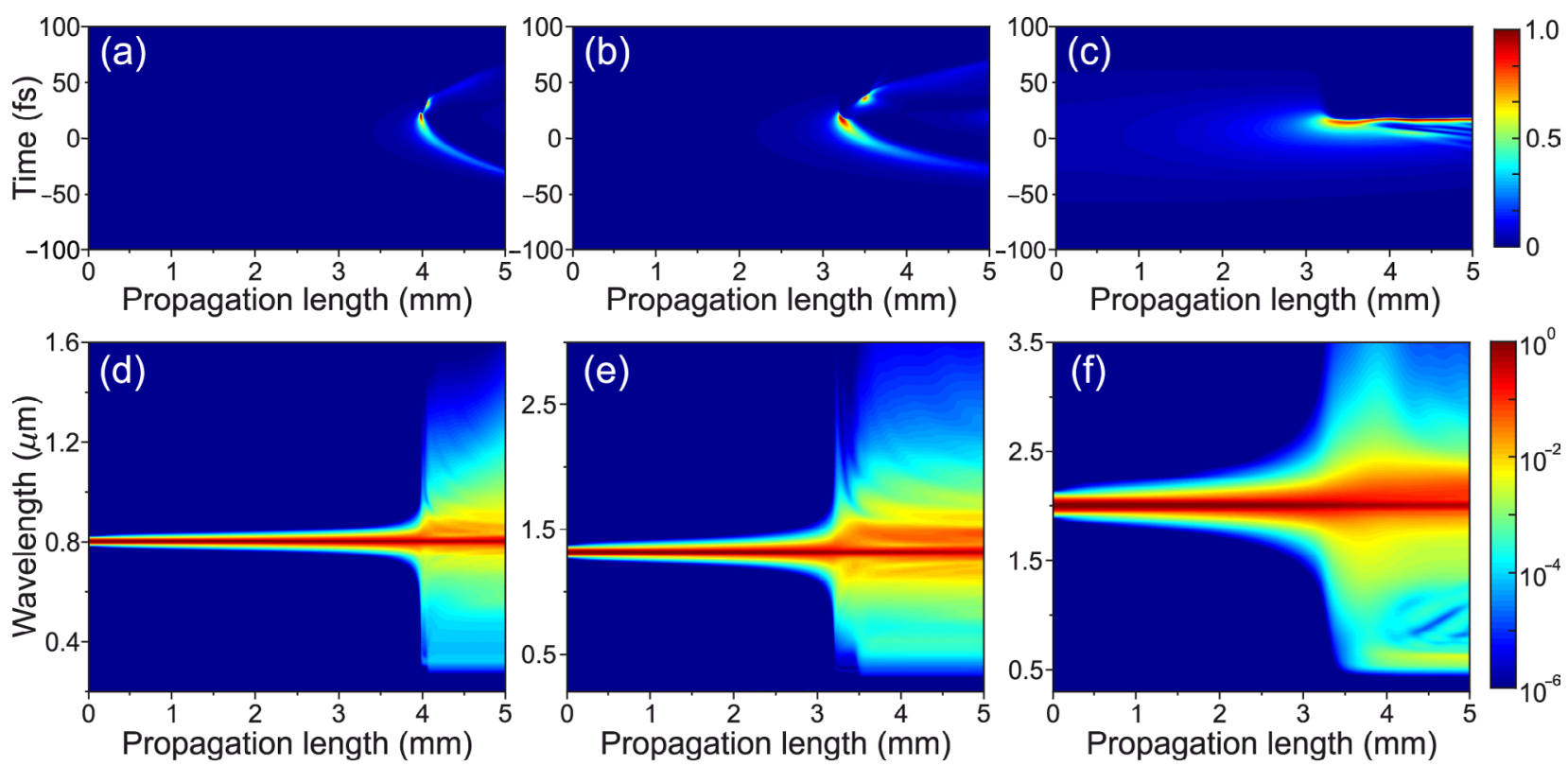

Fig. 3. Top row: numerically simulated temporal dynamics of $100 \mathrm{fs}$ laser pulses propagating in a sapphire crystal with the input wavelengths of (a) $800 \mathrm{~nm}$, (b) $1.3 \mu \mathrm{m}$ and (c) $2.0 \mu \mathrm{m}$, representing the filamentation regimes of normal, zero and anomalous GVD, respectively. Bottom row shows the corresponding spectral dynamics. Notice how the spectral broadening in the regimes of normal and zero GVD is associated with the pulse splitting, and the spectral broadening in the regime of anomalous GVD is associated with pulse self-compression. 
the intensities of split sub-pulses [69], while a detailed amplitude structure and phase information of split sub-pulses was retrieved from frequency resolved optical gating (FROG) [70], eventually establishing a general link between pulse splitting and SC generation [7].

These findings laid the basis of the pulse-splitting-based temporal scenario of SC generation in normally dispersive media [72]. As self-phase modulation broadens the pulse spectrum and produces nonlinear frequency modulation (chirp), in which red-shifted and blue-shifted frequencies are generated at the leading and trailing parts of the pulse, respectively, the pulse splitting at the nonlinear focus produces two sub-pulses with shifted carrier frequencies. Because of that, the sub-pulses move in opposite directions in the frame of the input pulse, as illustrated in Fig. 3(a). Pulse splitting is immediately followed by an explosive broadening of the spectrum (Fig. 3(d)), which is produced by the self-steepening effect. The latter effect originates from velocity differences between the pulse peak and the tails of pulses due to the refractive index dependence on the intensity, and so induces sharp intensity gradients (optical shocks) in the temporal profiles of sub-pulses.

Split sub-pulses experience rather different selfsteepenings, which quantitatively explain the asymmetry in experimentally measured shapes of the SC spectra. In the near-infrared spectral range, under typical focusing conditions, a particularly steep edge is formed at the trailing front of the trailing sub-pulse, giving rise to a broad blue-shifted pedestal in the SC spectrum. In contrast, self-steepening of the leading front of the leading sub-pulse is much less, resulting in a rather modest red-shifted spectral broadening. Connections between the leading and trailing sub-pulses, and the red-shifted and blue-shifted spectral broadenings were verified experimentally by measuring the spectral content of split sub-pulses [73]. The universality of the pulsesplitting-based scenario of SC generation in normally dispersive media is confirmed by an apparent similarity of the SC spectral shapes generated in various nonlinear media.

\subsection{Anomalous GVD}

A qualitatively different temporal scenario of the self-focusing and femtosecond filamentation was foreseen in the range of anomalous GVD, sug- gesting that the interplay between self-focusing, self-phase modulation and anomalous GVD may lead to a simultaneous shrinking of the input wave packet in spatial and temporal dimensions, giving rise to the formation of self-compressed three-dimensional (spatiotemporal) light bullets [74. Here new red-shifted and blue-shifted frequencies generated by the self-phase modulation on the ascending (leading) and descending (trailing) fronts of the pulse, respectively, are swept back to the peak of the pulse, instead of being dispersed as in the case of normal GVD. The feasibility of self-compressed objects was confirmed by numerical simulations using more realistic numerical models [75-77], which predicted that pulse self-compression down to a single optical cycle was potentially possible.

Development of high-peak-power near- and mid-infrared ultrashort-pulse laser sources, which were exclusively based on the optical parametric amplification, gave an experimental access to study filamentation phenomena in the range of anomalous GVD of wide-bandgap dielectrics and even semiconductors with zero GVD wavelengths located deeply in the mid-infrared. To this end, a remarkably (almost 10 times) increased length of the filaments [78] and ultrabroadband SC emission [79] was observed by launching femtosecond pulses at $1.55 \mu \mathrm{m}$ in fused silica. A more recent study demonstrated the filamentation of incident pulses with a much longer wavelength $(3.1 \mu \mathrm{m})$ in the YAG crystal, yielding more than a 3-octave spanning SC spectrum with the unprecedented wavelength coverage from the ultraviolet to the mid-infrared range [80]. Eventually, simultaneous time and space compression was demonstrated to favour a new type of filamentation, which produces quasistationary three-dimensional self-compressed light bullets that preserve a narrow beam diameter and a short pulsewidth over a considerable propagation distance in a nonlinear dispersive medium [81]. To this end, the formation of self-compressed spatiotemporal light bullets was experimentally observed in various nonlinear media, such as fused silica, sapphire and $\mathrm{BBO}$, and under a variety of operating conditions [82-86].

Figure 3(c) shows a numerical example illustrating formation and propagation dynamics of the self-compressed spatiotemporal light bullet in the sapphire crystal, which is accompanied 
by generation of ultrabroadband SC (Fig. 3(f)) emerging at the point where the pulse self-compression occurs.

\subsection{Zero GVD}

The zero GVD wavelengths of commonly used wide-bandgap dielectric materials lie in the infrared spectral range, in the wavelength interval between 1 and $2 \mu \mathrm{m}$ (see Table 1). To some extent, the near-zero GVD regime combines properties of both normal and anomalous GVD, and produces a more symmetric spectral broadening than in two previous propagation regimes [63]. However, in the time domain, the input pulse undergoes splitting at the nonlinear focus, as illustrated in Fig. 3(b), and the post-collapse dynamics are essentially similar to that observed in the case of normal GVD shown in Fig. 3(a). Experimental measurements show that pulse splitting prevails even in the case of weak anomalous GVD [87, where the amount of material dispersion is too small to compress the spectrally broadened pulse.

Figure 4 presents a comparison of the numerically simulated axial SC spectra generated by selffocusing and filamentation of $100 \mathrm{fs}$ pulses in a $4 \mathrm{~mm}$-thick sapphire crystal, in the regimes of normal (input wavelength $800 \mathrm{~nm})$, zero $(1.3 \mu \mathrm{m})$ and anomalous $(2.0 \mu \mathrm{m}) \mathrm{GVD}$.

\subsection{Conical emission}

Finally, the above filamentation and SC generation scenarios could be generalized by employing an effective three-wave mixing model, which provides a unified picture, connecting spectral broadening on the propagation axis with coloured conical emission [61, 62], which is perhaps the most striking and visually perceptible evidence of SC generation in bulk media. The spectral content and angular distribution of scattered waves satisfy phase matching conditions, which are defined by chromatic dispersion, thereby providing a particular dispersion-defined angular landscape of scattered frequencies [88]. Experimentally, these landscapes could be retrieved by measuring the SC spectrum with an imaging spectrometer [89]. Figures 5(a-c) show the experimentally measured angularly resolved SC spectra in water, which exhibit qualitatively different pat-

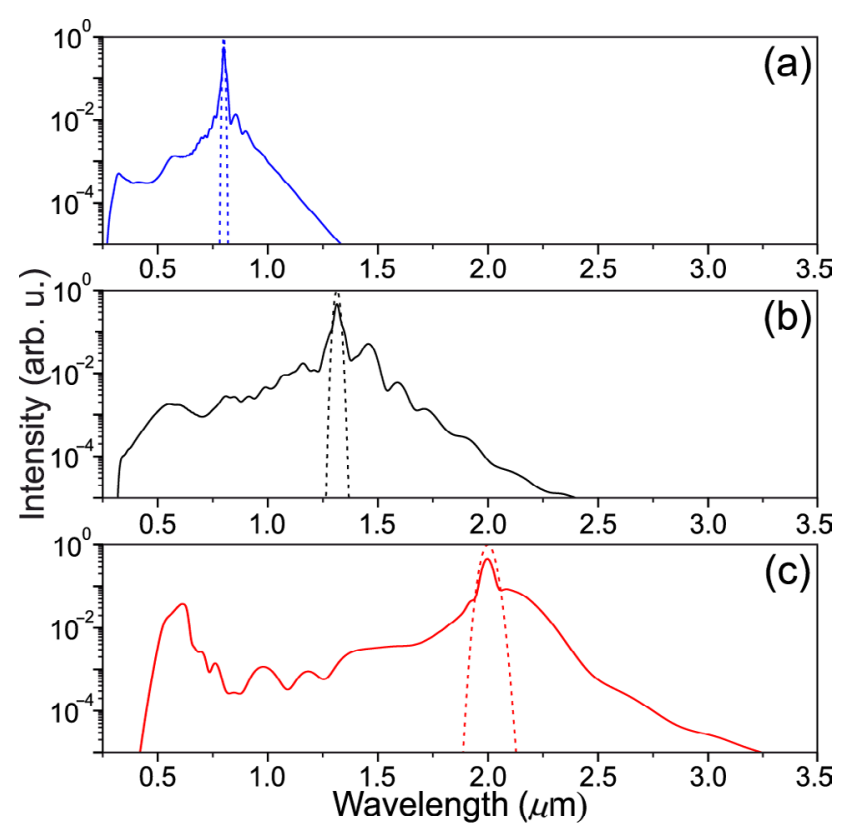

Fig. 4. Lineouts of the numerically simulated axial supercontinuum spectra after a $4 \mathrm{~mm}$-thick sapphire crystal in the cases of (a) normal GVD, (b) zero GVD and (c) anomalous GVD. The input spectra are shown by dashed curves.

terns of conical emission in the range of normal and anomalous GVD [90]. More specifically, in the range of normal GVD, off-axis (conical) tails emerge on both the blue and red-shifted sides of the input wavelength, forming a distinct $\mathrm{X}$-shaped pattern of conical emission, as shown in Fig. 5(a). In contrast, in the range of anomalous GVD, the conical emission pattern develops a multiple annular or $\mathrm{O}$-shaped structure around the input wavelength, as illustrated in Fig. 5(b). The entire angle-resolved SC spectrum produced by filamentation of $1055 \mathrm{~nm}$ laser pulses, whose wavelengths fall into the range of anomalous GVD of water, is presented in Fig. 5(c). It consists of multiple annular structures around the carrier wavelength and a distinct $\mathrm{V}$-shaped tail in the visible spectral range [91]. Figure 5(d) illustrates the entire angleresolved SC spectrum in sapphire, as generated by $800 \mathrm{~nm}$ pulses, in the range of normal GVD [92].

The shapes of angle-resolved SC spectra are universal for any other nonlinear medium. These findings led to the interpretation of femtosecond filaments as conical waves, assuming that the input wave packet will try to evolve toward a final stationary state that has the form of either an Xwave in the range of normal GVD or an $\mathrm{O}$-wave 
in the range of anomalous GVD. Nonlinear Xwaves [93, 94] and O-waves [95] are named because of their evident X-like and O-like shapes, respectively, which appear in both the near- and the far-fields. Moreover, the interpretation of light filaments in the framework of conical waves readily explains the distinctive propagation features of light filaments such as the sub-diffractive propagation in the free space [83, 92] and self-reconstruction after hitting physical obstacles [96-98], which are universal and regardless of the sign of material GVD, and which were verified experimentally as well. Therefore all subsequent features of the filament propagation in the regime of normal GVD, i.e. pulse splitting, conical emission and any nonlinear interactions, may be interpreted assuming the pulses as spontaneously occurring nonlinear $\mathrm{X}$-waves [99]. Consequently, the formation and propagation features of spatiotemporal light bullets in the regime of anomalous GVD may be interpreted in terms of nonlinear $\mathrm{O}$-waves [83].

\section{Numerical models}

A standard numerical model for the nonlinear propagation and filamentation of an intense femtosecond laser pulse in a transparent dielectric medium with cubic nonlinearity is based on solving the paraxial unidirectional propagation equation with cylindrical symmetry for the nonlinear pulse envelope coupled with rate equations describing the generation of free electrons by optical field ionization. In the most general case, the pulse propagation is described with the scalar equation for the transverse component of a linearly polarized electric field. Unidirectional propagation equations can be written in the form of generalized canonical equations in the spectral domain for the electrical field $\hat{E} \equiv \hat{E}\left(\omega, k_{x}, k_{y}, z\right)$ 100, 101]:

$$
\frac{\partial \hat{E}}{\partial z}=\mathrm{i} K\left(\omega, k_{x}, k_{y}\right) \hat{E}+\mathrm{i} Q\left(\omega, k_{x}, k_{y}\right) \frac{\hat{P}_{\mathrm{NL}}}{\epsilon_{0}} .
$$

Here $K\left(\omega, k_{x}, k_{y}\right) \equiv \sqrt{\omega^{2} n^{2}(\omega) / c^{2}-k_{x}^{2}-k_{y}^{2}} \quad$ represents the propagation constant for the modal components of the electric field, $n(\omega)$ denotes the linear refractive index of the medium, $c$ is the speed of light in a vacuum, $\epsilon_{0}$ is the permittivity of free space and $Q\left(\omega, k_{x}, k_{y}\right) \equiv \omega^{2} / 2 c^{2} K\left(\omega, k_{x}, k_{y}\right)$. The nonlinear polarization $P_{\mathrm{NL}}(t, x, y, z)$, whose
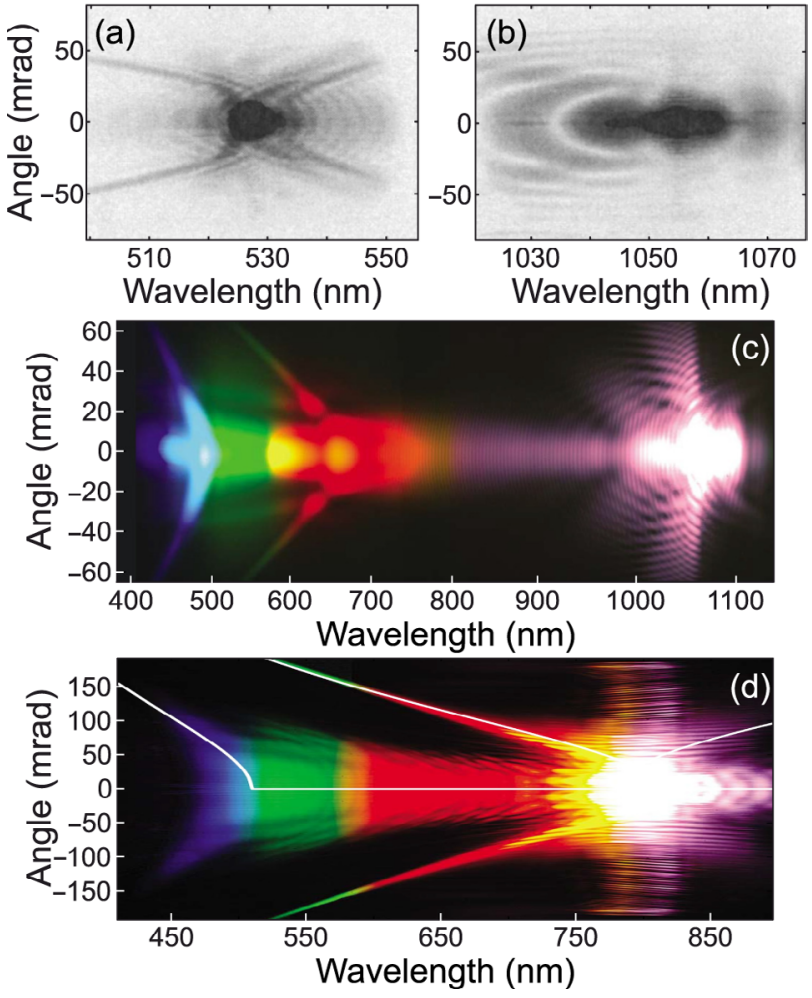

Fig. 5. Experimentally measured angle-resolved spectra around the incident wavelengths of $527 \mathrm{~nm}$ and $1.055 \mu \mathrm{m}$ that fall into the ranges of (a) normal GVD, (b) anomalous GVD of water, respectively. Adapted from [90]. (c) The entire angle-resolved SC spectrum in water as excited with $1.055 \mu \mathrm{m}$ input pulses. Adapted from [91]. (d) The angle-resolved SC spectrum as excited with $800 \mathrm{~nm}$ input pulses in sapphire. White solid curves indicate the best fits obtained using the X-wave relation. Adapted from [92].

spectral representation reads as $\hat{P}_{\mathrm{NL}}\left(\omega, k_{x}, k_{y}, z\right)$, describes the nonlinear response of the material and takes the form of constitutive relations linking $P_{\mathrm{NL}}(t, x, y, z)$ to the electric field $E(t, x, y, z)$. Expressing the propagation equation in the canonical form has the main advantage of generality, as Eq. (6) encompasses all unidirectional scalar propagation models that can be derived under various approximations, and can be simulated by means of a single algorithm [101]. $E(t, x, y, z)$ represents the electric field in the case of the carrier-resolving propagation equation, or the envelope of the laser pulse if the slowly varying envelope approximation is valid, i.e. if the pulse duration is much longer than the optical cycle. Note that the vectorial unidirectional pulse propagation can also be modelled by Eq. (6), for instance, in tight focusing 
conditions, provided $\hat{E}\left(\omega, k_{x}, k_{y}, z\right)$ is replaced by an appropriate component of the Hertz potential [102].

The functional forms of $K$ and $Q$ are quite general even if slightly different forms may be in use to reflect various approximations. For example, the paraxial approximation amounts to performing a small $\left(k_{x}^{2}+k_{y}^{2}\right)$-expansion of $K$ and $Q$ as $K \sim k(\omega)-\left(k_{x}^{2}+k_{y}^{2}\right) / 2 k(\omega)$ and $Q \sim \omega / 2 c n(\omega)$, where $k(\omega) \equiv n(\omega) \omega / c$. In this case, Eq. (6) is the spectral representation of the forward Maxwell equation [103]. For an envelope propagation equation, $k(\omega)$ is usually expanded as a Taylor series around the pulse carrier frequency $\omega_{0}$ :

$$
k(\omega) \sim k_{0}+k_{0}^{\prime}\left(\omega-\omega_{0}\right)+\frac{k_{0}^{\prime \prime}}{2}\left(\omega-\omega_{0}\right)^{2}+\frac{k_{0}^{\prime \prime \prime}}{3 !}\left(\omega-\omega_{0}\right)^{3}+\ldots .
$$

Combination of the paraxial approximation with the slowly varying envelope approximation, truncation of the Taylor series to the second order (and using $\left.K \sim k(\omega)-\left(k_{x}^{2}+k_{y}^{2}\right) / 2 k_{0}, Q \sim \omega_{0} / 2 c n_{0}\right)$ and factorization of the carrier wave $\exp \left(-\mathrm{i} \omega_{0} t\right)$ lead to the family of nonlinear Schrödinger propagation equations:

$$
\frac{\partial E}{\partial z}+k_{0}^{\prime} \frac{\partial E}{\partial t}=\frac{\mathrm{i}}{2 k_{0}} \Delta_{\perp} E-\mathrm{i} \frac{k_{0}^{\prime \prime}}{2} \frac{\partial^{2} E}{\partial t^{2}}+\mathrm{i} \frac{\omega_{0}}{2 c n_{0}} \frac{P_{\mathrm{NL}}}{\epsilon_{0}} .
$$

Including higher order terms in the Taylor series and keeping the first order in $\omega-\omega_{0}$ corrective terms in $k(\omega)$ and $Q(\omega)$, the family of nonlinear envelope equations is obtained 104.

The term $k_{0}^{\prime} \partial E / \partial t$ on the left-hand side of Eq. (8) represents a unidirectional propagation of the pulse at the group velocity $k_{0}^{\prime-1}$. The first term on the right-hand side accounts for diffraction, where $\Delta_{\perp}$ denotes the Laplacian with respect to the coordinates $x$ and $y$ in the transverse plane. The second term accounts for the group velocity dispersion. The sign of the coefficient $k_{0}^{\prime \prime} \equiv \mathrm{d}^{2} k /$ $\left.\mathrm{d} \omega^{2}\right|_{\omega_{0}}$ determines whether dispersion is normal $\left(k_{0}^{\prime \prime}>0\right)$ or anomalous $\left(k_{0}^{\prime \prime}<0\right)$. Note that its sign also determines the hyperbolic or elliptic nature of space and time couplings in Eq. (8), which are responsible for spatiotemporal reshaping into nonlinear $\mathrm{X}$ - and $\mathrm{O}$-waves, respectively, as discussed in the previous section.

The nonlinear polarization is expressed in the form that distinguishes the responses of bound and free electrons:

$$
P_{\mathrm{NL}}=P_{\text {bound }}+P_{\text {free }}
$$

The response of bound electrons can be modelled via instantaneous Kerr or delayed Raman responses, whose fractional contributions are denoted by $\alpha$ and $1-\alpha$, respectively, assuming that the total cubic susceptibility is constant within the frequency range of interest

$$
\begin{aligned}
& P_{\text {bound }}=\epsilon_{0} \chi^{(3)}\left(\int_{-\infty}^{t} R\left(t-t^{\prime}\right) E^{2}\left(t^{\prime}\right) \mathrm{d} t^{\prime}\right) E(t), \\
& R(t)=(1-\alpha) \delta(t)+\alpha H(t) \Omega \exp (-\Gamma t) \sin (\Lambda t),
\end{aligned}
$$

where $\delta(t)$ is the Dirac delta-function, $H(t)$ is the Heaviside step-function, and $\Omega=\frac{\Lambda^{2}+\Gamma^{2}}{\Lambda}$, with $\Gamma$ and $\Lambda$ being the characteristic frequencies for the Raman response of the dielectric medium. The nonlinear polarization induced by bound electrons, $P_{\text {bound }}$, is responsible for two of the most relevant effects in filamentation and supercontinuum generation: self-focusing and self-phase modulation (discussed in the previous section). For a carrier resolving model, this term also accounts for the third harmonic generation and the generation of other low order odd harmonics by cascaded four-wave mixing. For an envelope propagation model designed to simulate the supercontinuum generation over a limited spectral region, if no spectral overlap with the third harmonic is expected, it is sufficient to replace the field squared $E^{2}(t)$ by the squared modulus of the complex envelope $|E|^{2}$ in the nonlinear response.

Free electron generation can be modelled by considering a simple rate equation describing the evolution of the plasma density $\rho_{\mathrm{e}}$ :

$$
\partial_{\mathrm{t}} \rho_{\mathrm{e}}=W(I)\left(\rho_{\mathrm{nt}}-\rho_{\mathrm{e}}\right)+\frac{\sigma}{U_{\mathrm{g}}} \rho_{\mathrm{e}} I+\left.\partial_{t} \rho_{\mathrm{e}}\right|_{\mathrm{rec}} \cdot
$$

Here $W(I)$ denotes the intensity dependent photoionization rate, $\rho_{\mathrm{nt}}$ is the neutral density in the valence band, $U_{\mathrm{g}}$ is the energy gap between the valence and the conduction band, and $\sigma$ is the cross section for inverse Bremsstrahlung. The first term on the right-hand side of Eq. (12) stands for photo-ionization, the second term stands for avalanche ionization, while the third term stands for recombination. The response of free electrons is conveniently described by a current, acting as 
a source term in the propagation equation. The total current is linked to the nonlinear polarization induced by free electrons, $P_{\text {free }}$, and is contributed by two components:

$$
\begin{aligned}
& \partial_{t} P_{\text {free }}=J_{\mathrm{e}}+J_{\text {loss' }} \\
& \partial_{t} J_{\mathrm{e}}+v_{\mathrm{c}} J_{\mathrm{e}}=\frac{e^{2}}{m} \rho_{\mathrm{e}} E, \\
& J_{\text {loss }}=\epsilon_{0} c n_{0} W(I) U_{g}\left(\rho_{\mathrm{nt}}-\rho_{\mathrm{e}}\right) E .
\end{aligned}
$$

Equation (14) is based on the Drude model and describes the motion of electrons accelerated by the laser field, undergoing friction at the rate $v_{c}$ due to collisions with ions. $J_{\text {loss }}$ is responsible for the loss of energy necessary to ionize the medium and is described by the phenomenological equation, Eq. (15). As a source term in the propagation Eq. (6), $J$ is responsible for plasma defocusing and plasma absorption.

For further details, an interested reader may refer to a didactically excellent review [101], which provides the necessary theoretical background, basic building blocks and tools to perform numerical simulation with proper understanding of the underlying physical effects. A more recent review [105] provides a classification of various approaches to optical field evolution equations, light-matter interaction models and methods that can be integrated with time- and space-resolved simulations encompassing a wide range of realistic experimental scenarios.

\section{Practical considerations}

Although femtosecond filamentation and SC generation emerge from a complex interplay among linear (diffraction and GVD) and nonlinear effects (self-focusing, self-phase modulation, pulse splitting, pulse-front steepening, generation of optical shocks, multiphoton absorption and free electron plasma generation), the practical setup for supercontinuum generation is amazingly simple. It involves just a focusing lens, a piece of suitable nonlinear material and a collimating lens. In general, the laser wavelength and the value of the nonlinear index of refraction define the critical power for self-focusing (see Eq. (1)), which in turn sets the lowest margin of the input energy required to generate a light filament. In the near-infrared spectral range, the typical values of $P_{\text {cr }}$ in wide-bandgap dielectric media are of the order of several MW, that are easily achieved with femtosecond pulses of energies in the range from few microjoules to few hundreds of nanojoules. Typically, the incident beam is externally focused to a diameter of $30-100 \mu \mathrm{m}$, which guarantees the location of the nonlinear focus inside the nonlinear medium of several mm thickness. Under suitable focusing conditions, $\mathrm{SC}$ is excited with the input

Table 1. Linear and nonlinear parameters of basic dielectric media used for supercontinuum generation. $U_{\mathrm{g}}$ is the energy bandgap, the transmission range is defined at $10 \%$ transmission level in a $1 \mathrm{~mm}$ thick sample, $n_{0}$ and $n_{2}$ are linear and nonlinear refractive indexes, respectively, and are given for $\lambda=800 \mathrm{~nm}, \lambda_{0}$ is the zero GVD wavelength.

\begin{tabular}{ccccccc}
\hline Material & $U_{g}, \mathrm{eV}$ & Transmittance, $\mu \mathrm{m}$ & $n_{2}, \times 10^{-16} \mathrm{~cm}^{2} / \mathrm{W}$ & $n_{0}$ & $\lambda_{0}, \mu \mathrm{m}$ \\
\hline $\mathrm{LiF}$ & $13.6[106]$ & $0.12-6.6[106]$ & $0.81[107]$ & $1.39[108]$ & $1.23[108]$ \\
\hline $\mathrm{CaF}_{2}$ & $10[106]$ & $0.12-10[106]$ & $1.3[109]$ & $1.43[110]$ & $1.55[110]$ \\
\hline $\mathrm{Al}_{2} \mathrm{O}_{3}$ & $9.9[106]$ & $0.19-5.2[106]$ & $3.1[111]$ & $1.76[106]$ & $1.31[106]$ \\
\hline $\mathrm{BaF}_{2}$ & $9.1[106]$ & $0.14-13[106]$ & $1.91[109]$ & $1.47[110]$ & $1.93[110]$ \\
\hline $\mathrm{SiO}_{2}(\mathrm{FS})$ & $9.0[112]$ & $0.18-3.5[106]$ & $2.4[113]$ & $1.45[114]$ & $1.27[114]$ \\
\hline $\mathrm{KDP}$ & $7.0[115]$ & $0.18-1.55[115]$ & $2.0[115]$ & $1.50[116]$ & $0.98[116]$ \\
\hline $\mathrm{H}_{2} \mathrm{O}$ & $6.9[117]$ & $0.18-1.3[106]$ & $5.7[118]$ & $1.33[119]$ & $1.0[119]$ \\
\hline $\mathrm{YAG}$ & $6.5[120]$ & $0.21-5.2[106]$ & $6.2[109]$ & $1.82[121]$ & $1.60[121]$ \\
\hline$\beta-\mathrm{BBO}$ & $6.2[115]$ & $0.19-3.5[115]$ & $5.2[122]$ & $1.66[123]$ & $1.49[123]$ \\
\hline $\mathrm{BK} 7$ & $4.28[124]$ & $0.3-2.5[125]$ & $3.75[118]$ & $1.51[125]$ & $1.32[125]$ \\
\hline $\mathrm{KGW}$ & $4.05[126]$ & $0.3-5[127]$ & $11[128]$ & $2.02[129]$ & $2.2[129]$ \\
\hline $\mathrm{YVO}_{4}$ & $3.8[130]$ & $0.35-4.8[106]$ & $15[128]$ & $1.97[106]$ & \\
\hline
\end{tabular}


beam powers which exceed $P_{\text {cr }}$ just by several tens of percent. Slightly converging or diverging laser beams may be also in use; then the position of the nonlinear focus is defined by

$$
\frac{1}{z_{\mathrm{sf}}^{\prime}}=\frac{1}{z_{\mathrm{sf}}}+\frac{1}{f}
$$

where $f$ is the focal length of the focusing lens. Notice that with converging the input beam the nonlinear focus occurs before the geometrical focus. In the case of diverging the input beam (the geometrical focus is located before the input face of the nonlinear medium), the input pulse power should exceed $P_{\text {cr }}$ by several times, since the self-focusing effect should overcome the divergence of the beam.

Relevant linear and nonlinear parameters for widely used nonlinear dielectric media are provided in Table 1. Figure 6 illustrates the calculated critical power for self-focusing in various dielectric materials at the input wavelengths of 800 and $1030 \mathrm{~nm}$, which are emitted by commonly used Ti:sapphire and $\mathrm{Yb}$-doped femtosecond lasers, respectively. However, to generate stable and reproducible SC, some important issues of laser-matter interaction, and femtosecond filamentation in particular, should be taken into consideration. These practical issues are universal and hold for any nonlinear medium and at any input wavelength in the optical range. First of

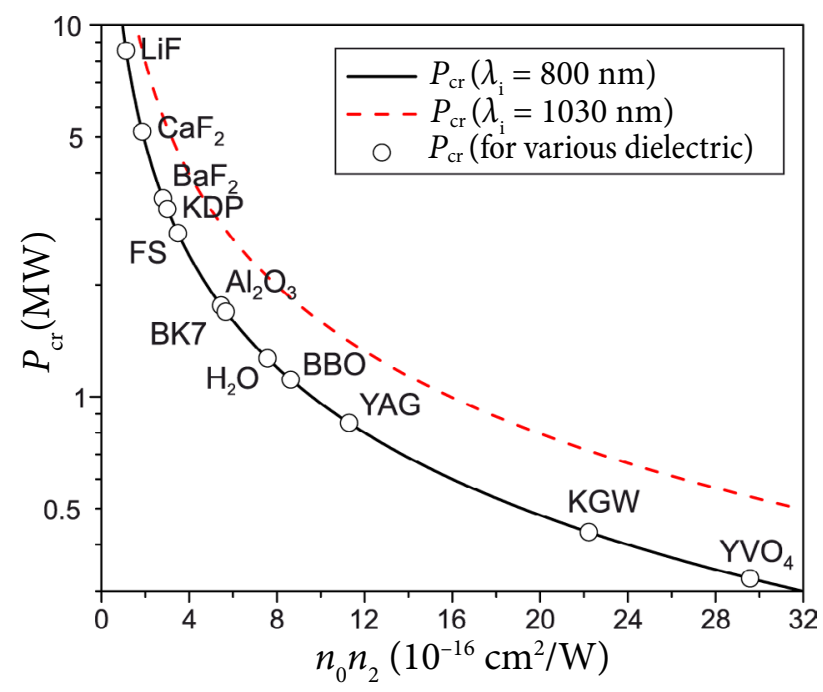

Fig. 6. Critical power for self-focusing calculated for Ti:sapphire ( $800 \mathrm{~nm}$, solid curve) and Yb-doped (1030 nm, dashed curve) laser wavelengths. Circles denote the critical power for self-focusing at $800 \mathrm{~nm}$ in various dielectric materials. all, the optical damage of the medium is the major limiting factor in SC generation, therefore the external focusing condition (the numerical aperture, NA) should be carefully chosen [131, 132]. Figure 7 shows the experimentally measured threshold energies for SC generation and optical damage in fused silica as functions of the numerical aperture [132].

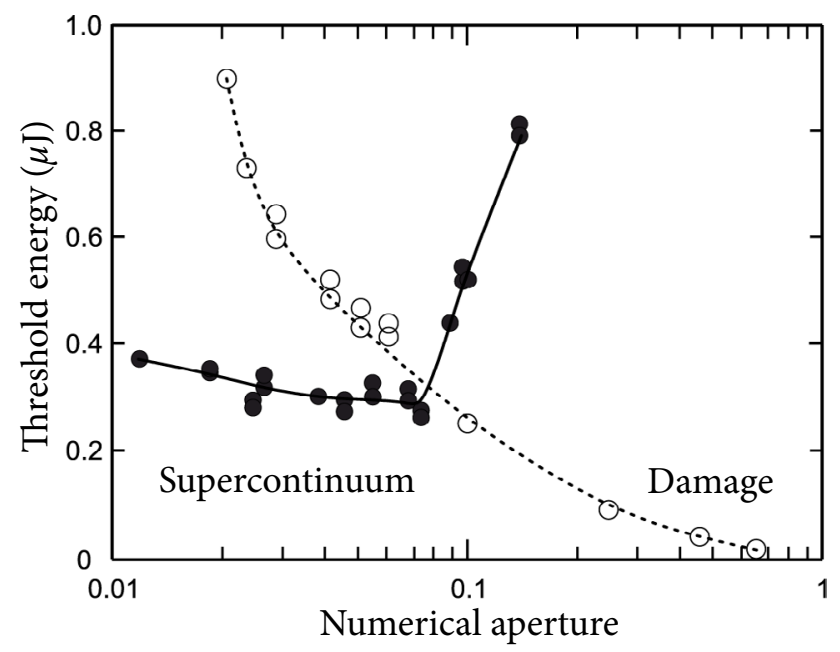

Fig. 7. Energy thresholds for damage (open circles) and supercontinuum generation (filled circles) in UV-grade fused silica with $60 \mathrm{fs}, 800 \mathrm{~nm}$ laser pulses versus NA. The curves serve as guides for the eye. Adapted from [132].

In the high-NA regime (NA $>0.25$ ), the optical damage occurs for the input pulse energies below the energy corresponding to the critical power of self-focusing, so no SC generation under such focusing condition is observed. Most of the pulse energy is thus deposited into the material at the focal volume through multiphoton absorption and subsequent linear absorption by the plasma: a laser-matter interaction regime that is exploited for micromachining of transparent bulk materials. In the NA range from 0.15 to 0.05 , the threshold energies for $\mathrm{SC}$ generation and optical damage are very close. In particular, for NA $<0.1, \mathrm{SC}$ is generated without the optical damage in a single shot regime, however, the damage accumulates under a multiple shot exposure, causing SC to disappear over time.

Finally, with NA below 0.05 , it is still possible to damage the medium, but only at the input pulse energies significantly above the threshold for SC generation, hence constituting the "safe" operating condition for SC generation. Moreover, 
experiments show that the loose focusing condition facilitates the enhanced red-shifted broadening of the SC spectrum [26], stemming from the increased nonlinear propagation of the leading sub-pulse, which preserves a steep ascending front [133].

However, for numerical apertures below 0.05, corresponding to the loose focusing condition, a sufficiently long nonlinear medium and the input beam power exceeding $P_{\text {cr }}$ by several times, femtosecond filament may undergo recurrent self-focusing cycles, which could be directly visualized by monitoring the multiphoton absorptioninduced fluorescence traces with a specific colour appearance, which depends on the material, as detected from a side-view of the nonlinear medium (see, e.g. [134-136]). The general interpretation of focusing/refocusing cycles is based on the socalled dynamic spatial replenishment scenario, which assumes alternating cycles of self-focusing due to the Kerr effect and self-defocusing due to free electron plasma and which was originally proposed to explain the illusion of long-distance selfguided propagation of high-power pulses in gaseous media [137]. A more recent experimental and numerical study of the full spatiotemporal evolution of light filaments versus the propagation distance in water unveiled the intimate connections between complex propagation effects: focusing and refocusing cycles, nonlinear absorption, pulse splitting and replenishment, supercontinuum generation and conical emission [138]. More specifically, whenever the self-focusing wave packet (the ultrashort pulsed laser beam) approaches the nonlinear focus, multiphoton absorption attenuates its central part, which after the pulse splitting is reshaped into a ring-like structure. With further propagation the leading and trailing sub-pulses separate before the light contained in the ring refocuses and replenishes the pulse on the propagation axis, as illustrated in Fig. 8(b). If the power of the replenished pulse is above critical, the replenished pulse undergoes another selffocusing cycle, which results in pulse splitting at the secondary nonlinear focus. The secondary pulse splitting produces yet another portion of the SC, and the resulting SC spectrum and conical emission pattern develop a periodic modulation, due to interference between the primary and the secondary split sub-pulses.

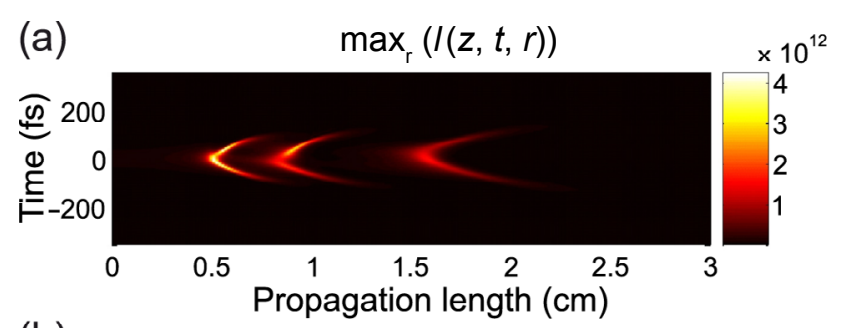

(b)

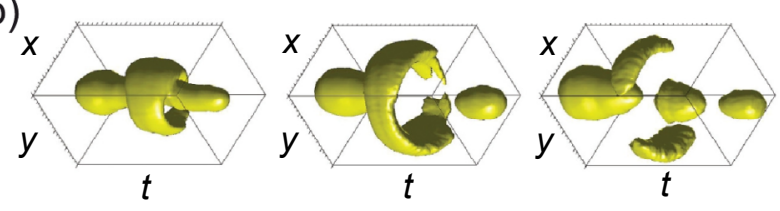

Fig. 8. (a) Numerical simulation of temporal profiles for the axial intensity distribution illustrating focusing/refocusing cycles and recurrent pulse splitting events of $90 \mathrm{fs}$ ultraviolet $(400 \mathrm{~nm})$ laser pulse with $400 \mathrm{~nJ}$ energy propagating in water. (b) Experimentally measured spatiotemporal $(x, y, t)$ profiles of a filament at various stages of propagation after the first nonlinear focus. Adapted from [138].

After the tertiary splitting event, the modulation in the SC spectrum has beatings contributed by the occurrence of tertiary split sub-pulses etc. Focusing/refocusing cycles may repeat as long as the replenished pulse contains the power still above critical. However, each refocusing cycle is followed by a sudden decrease in the transmittance and therefore the entire beam will continuously lose energy during propagation, and eventually a linear propagation regime is resumed.

Figure 9 illustrates the above considerations by comparing visually perceptible filamentation features and SC spectra in a YAG crystal, as generated with $100 \mathrm{fs}, 800 \mathrm{~nm}$ input pulses with energies of $310 \mathrm{~nJ}$ (peak power of $\left.3.6 P_{\mathrm{cr}}\right)$ and $560 \mathrm{~nJ}\left(6.6 P_{\mathrm{cr}}\right)$, that induce a single self-focusing event and refocusing of the filament, respectively. The single self-focusing event is visualized by a gradually decaying plasma fluorescence trace, whose most intense part indicates the position of the nonlinear focus, as shown in Fig. 9(a), and produces a featureless far-field pattern of SC emission (Fig. $9(\mathrm{c})$ ) and a smooth SC spectrum (Fig. 9(e)). In contrast, the refocusing of the filament emerges as a double-peaked plasma fluorescence trace, as shown in Fig. 9 (b), and results in the occurrence of modulation in the outer part of the far-field pattern of SC emission (Fig. 9(d)) and periodic modulation of the SC spectrum (Fig. $9(\mathrm{f})$ ). These indications are very important from a practical point of view, 

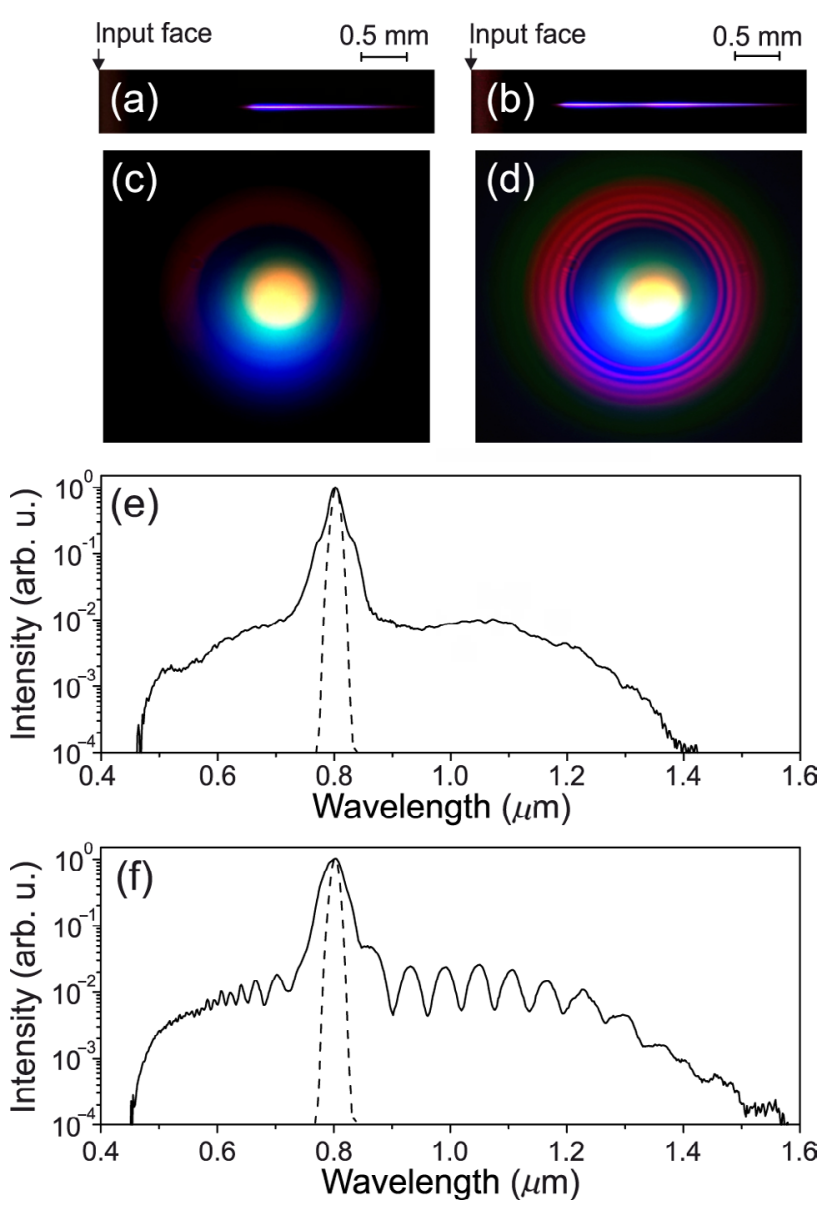

Fig. 9. Plasma fluorescence traces in a YAG crystal induced by self-focusing of $100 \mathrm{fs}, 800 \mathrm{~nm}$ input pulses with energies of (a) $310 \mathrm{~nJ}$ and (b) $560 \mathrm{~nJ}$, that induce a single self-focusing event and refocusing of the filament, respectively. (c) and (d) show the corresponding far-field patterns of SC emission, (e) and (f) show the corresponding SC spectra. Dashed curves show the input pulse spectrum. See the text for details.

since they allow us to easily identify the recurrent collapse and pulse splitting events and optimize the operating conditions for SC generation without employing complex experimental measurements.

In the filamentation regime in the range of anomalous GVD, where pulse self-compression rather than splitting at the nonlinear focus takes place, the recurrent self-focusing cycles manifest themselves in a similar manner [85, 139]. The refocusing event was shown to produce splitting of the light bullet at the secondary nonlinear focus [84], while multiple refocusing cycles were demonstrated to yield the formation of a sequence of quasi-periodical light bullets, each of them resulting in ejection of a new portion of the SC.
Finally, further increase of the input pulse energy leads to beam break-up into several or multiple filaments, which emerge from random amplitude and phase fluctuations inevitably present in real input beams and pulses. The intensity distributions of multiple filaments emerge in the form of regular or irregular patterns, that are governed by the input beam size, symmetry and smoothness (see, e.g. [140-142]). Although the multifilamentation regime creates an illusion of energy scaling of SC, each individual filament is subject to a combined effect of the intensity clamping [60] and the dispersion landscape of the material [61, 62], hence no additional spectral broadening with increasing the input power is achieved. Moreover, beam break-up into multiple filaments deteriorates the spatial uniformity of the beam and the temporal structure of the pulse, and eventually induces a considerable depolarization at various parts of the SC spectrum [24, 25.

\section{Supercontinuum generation in wide-bandgap dielectrics}

Figure 10 presents a graphical summary of the most relevant experimental results on SC generation in wide-bandgap dielectric media, as achieved with various femtosecond laser sources spanning pump wavelengths from the ultraviolet to the mid-infrared range. The experimental details are provided below.

\subsection{Glasses}

Silica-based glasses are multifunctional optical materials, which find diverse applications in contemporary optical science and technology. Thanks to a large bandgap and a reasonably large nonlinear refractive index, as combined with high optical and mechanical quality, fused silica serves as a standard nonlinear medium for studies of various fundamental and practical aspects of laser-matter interaction, such as self-focusing, self-phase modulation, photoionization, free-carrier absorption, carrier-carrier interaction, and eventually, mechanisms of the optical damage $[112,143,144]$. Therefore it is of no surprise that experiments in fused silica provided a valued practical knowledge, which allowed optimization of experimental schemes for SC generation. 


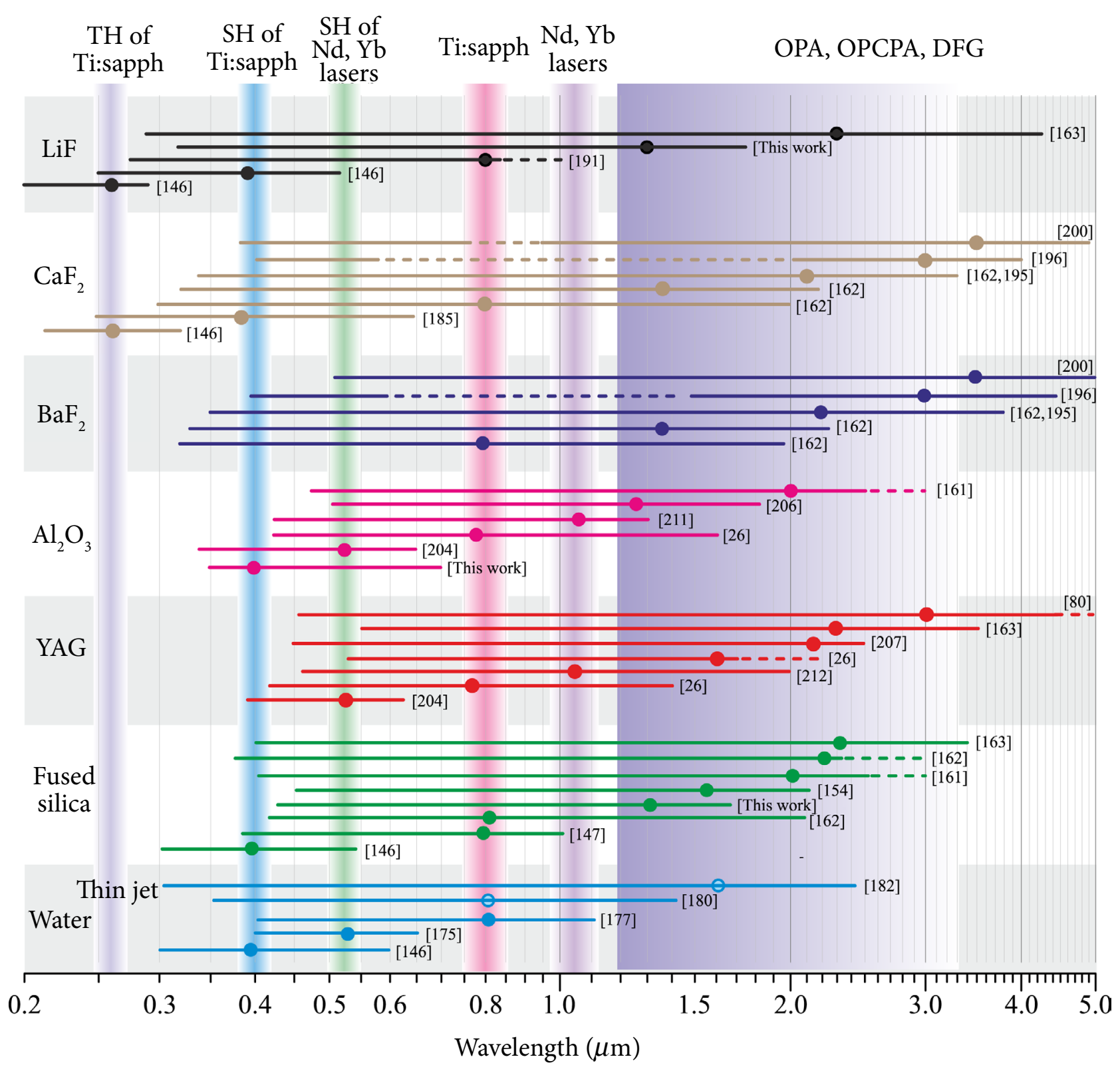

Fig. 10. Summary of the experimentally measured supercontinuum spectra in commonly used wide-bandgap dielectric media. The bold circles mark the pump wavelengths. Dashed lines indicate the spectral ranges into which further spectral broadening was expected, but not measured because of a limited detection range. Vertical colour (online) shading and labels on top indicate the laser sources: $\mathrm{TH}$, third harmonic; $\mathrm{SH}$, second harmonic; OPA, optical parametric amplifier; OPCPA, optical parametric chirped pulse amplifier; DFG, difference frequency generator.

Spectral broadening and SC generation in fused silica was investigated within a wide range of pump wavelengths, from the deep-ultraviolet to the mid-infrared range. In the deep-ultraviolet range, only a very slight spectral broadening around the carrier wavelength was observed with femtosecond pulses at $248 \mathrm{~nm}$ from an amplified excimer laser [145] and with pulses at $262 \mathrm{~nm}$ produced by frequency tripling of the Ti:sapphire laser output [146]. A more noticeable, but still modest spectral broadening in fused silica and BK7 glass was reported with the second harmonic pulses at $393 \mathrm{~nm}$ of the Ti:sapphire laser [146]. With the pump wavelength located in the visible spectral range, the SC spectrum from 415 to $720 \mathrm{~nm}$ was generated with $250 \mathrm{fs}$ pulses at $594 \mathrm{~nm}$ from a rhodamine $6 \mathrm{G}$ dye laser [11].

Many experiments on SC generation in fused silica were performed with a standard near-infrared pumping, using the fundamental harmonic of 
the Ti:sapphire laser and with the input pulsewidths of $100 \mathrm{fs}$ and shorter. A typical SC spectrum produced in fused silica extends from 390 to $1000 \mathrm{~nm}$, as reported under various experimental conditions [131, 132, 146-149]. Although similar, but somewhat narrower SC spectra were generated in BK7 [146, 150, 151] and ZK7 [152] glasses, which possess smaller bandgaps. Filamentation of laser pulses with longer wavelengths, as delivered by optical parametric amplifiers, yielded considerably broader SC spectra, as reported with pump wavelengths close or slightly above the zero GVD points of fused silica and BK7 glass [79, 153, 154, which are located at 1.27 and $1.32 \mu \mathrm{m}$, respectively (see Table 1). Figure 11 shows an example of the SC spectrum generated at the near zero GVD point of fused silica (pump wavelength $1.3 \mu \mathrm{m}$ ).

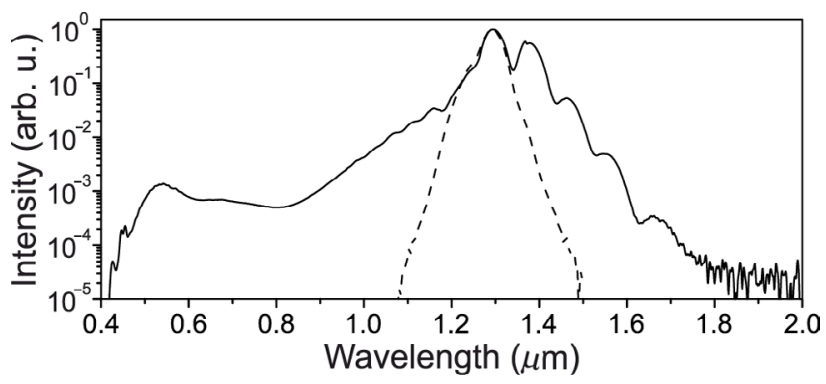

Fig. 11. Supercontinuum spectrum generated by filamentation of $100 \mathrm{fs}$ pulses with an energy of $1.9 \mu \mathrm{J}$ in a $3 \mathrm{~mm}$-long UV grade fused silica sample. The dashed curve shows the input pulse spectrum, with its central wavelength $(1.3 \mu \mathrm{m})$ close to the zero GVD point of fused silica.

SC generation experiments with pump wavelengths falling in the range of anomalous GVD of fused silica uncovered a number of universal features, which characterize the entire shape of the SC spectrum in the spectral-angular domain [155-157]. The angle-integrated as well as the axial SC spectra show an intense blue-shifted peak located in the visible range, which is identified as an axial component of the conical emission and whose blue-shift increases with increasing the wavelength of the driving pulse [157, 158]. Various aspects of the spectral broadening and SC generation, such as energy content, stability of the carrier envelope phase, etc., were studied in connection with formation and propagation dynamics of spatiotemporal light bullets [77, 159,
160. Experiments on self-focusing and filamentation in fused silica of few optical cycle pulses with carrier wavelengths of $2 \mu \mathrm{m}$ [161] and $2.2 \mu \mathrm{m}$ [162] reported ultrabroad SC spectra starting from 400 and $370 \mathrm{~nm}$, respectively, and extending to wavelengths greater than $2.5 \mu \mathrm{m}$. More recently, the recorded dynamics of spectral broadening in fused silica with the pump wavelength of $2.3 \mu \mathrm{m}$ versus the input pulse energy revealed that under given experimental conditions there exists the optimum pump pulse energy to obtain the broadest SC spectrum [163] (see Fig. 12(a)). Figure 12(b) shows the broadest SC spectrum generated with the optimum input pulse energy of $2.8 \mu \mathrm{J}$, providing a continuous wavelength coverage from $310 \mathrm{~nm}$ to $3.75 \mu \mathrm{m}$, which converts to 3.6 optical octaves.

Various non-silica glasses currently receive an increasing attention as potential candidates for SC
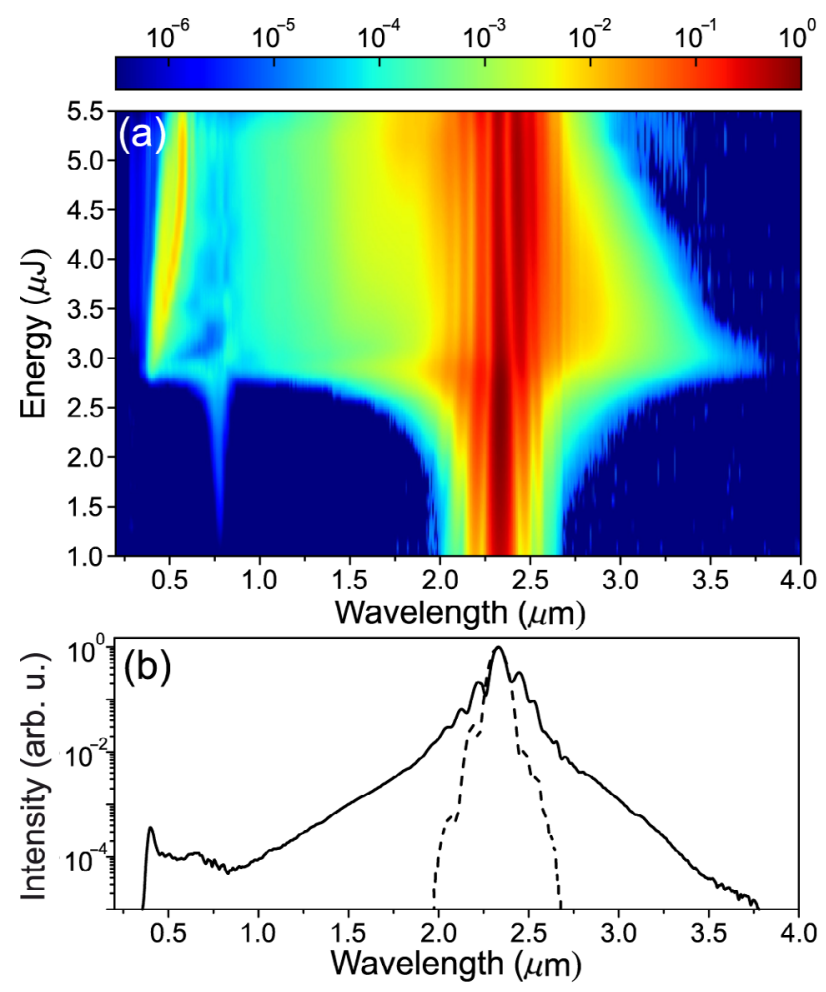

Fig. 12. (a) Spectral broadening of $100 \mathrm{fs}, 2.3 \mu \mathrm{m}$ laser pulse in a $3 \mathrm{~mm}$-long fused silica sample versus the input pulse energy. The spectral peak centred at $767 \mathrm{~nm}$, which appears before the onset of supercontinuum generation, is the third harmonic. (b) The broadest supercontinuum spectrum as measured with the input pulse energy of $2.8 \mu \mathrm{J}$. Notice an intense blue-shifted peak centred at $430 \mathrm{~nm}$. The input pulse spectrum is shown by a dashed curve. Adapted from [163]. 
generation. Using the pump pulses at $1.6 \mu \mathrm{m}$ from the optical parametric amplifier, the SC spanning wavelengths from 400 to $2800 \mathrm{~nm}$ were reported in lanthanum glass [164]. An impressive spectral broadening was observed in fluoride glass (ZBLAN) under similar pumping conditions; the authors generated an ultra-broadband, more than 5 octave-wide $\mathrm{SC}$, covering almost the entire transmission range of the material $(0.2-8.0 \mu \mathrm{m})$ [165]. The so-called soft glasses hold a great potential for SC generation in the mid-infrared spectral range. These materials are widely exploited in modern optical fibre technology, however, bulk soft glasses, such as tellurite and chalcogenide, also show very promising results. The SC spectra extending from the visible to $6 \mu \mathrm{m}$ and from the visible to $4 \mu \mathrm{m}$ were reported in tellurite glass using the pump pulses with central wavelengths of $1.6 \mu \mathrm{m}$ [166] and $2.05 \mu \mathrm{m}$ [167], respectively. A flat SC in the $2.5-7.5 \mu \mathrm{m}$ range was produced in bulk chalcogenide glass using the pump pulse with a central wavelength of $5.3 \mu \mathrm{m}$ [168]. The SC spectra with a remarkable mid-infrared coverage from 2.5 to $\sim 11 \mu \mathrm{m}$ were produced in $\mathrm{As}_{2} \mathrm{~S}_{3}$ and $\mathrm{GeS}_{3}$ bulk chalcogenide glass samples using 65 fs pulses at $4.8 \mu \mathrm{m}$ [169]. A more recent study reported on generation of the mid-infrared SC extending from 2.44 to $12 \mu \mathrm{m}$ in chalcogenide glass samples of various composition, as pumped with $70 \mathrm{fs}$ pulses of a wavelength tunable in the $3.75-5.0 \mu \mathrm{m}$ range [170].

\subsection{Water}

The absence of permanent optical damage and the possibility to easily vary the medium thickness makes liquids attractive media for many experiments in nonlinear optics. Water occupies an exceptional place among other liquids because of its technological and biomedical importance. Alongside fused silica, in many occasions water serves as a prototypical nonlinear medium for studies of ultrafast light-matter interactions. Systematic studies of the spectral broadening in water date back to the mid-1980s [171] and successfully continue in the femtosecond laser era, see [11, 172-174 for the early accounts on femtosecond SC generation in water and some other liquids.

Generation of femtosecond SC in water was performed with ultraviolet, visible and near infrared pump wavelengths. With the ultraviolet pump pulses generated by the frequency doubling of an amplified Ti:sapphire laser output, the measured SC spectra covered wavelength ranges of 290$530 \mathrm{~nm}$ [146] and 350-550 nm [138], as reported with pump wavelengths of 393 and $400 \mathrm{~nm}$, respectively, and under slightly different external focusing conditions. With the pump wavelengths in the visible spectral range, the SC spectrum from 400 to $650 \mathrm{~nm}$ was generated with $527 \mathrm{~nm}$ self-compressed femtosecond second harmonic pulses from the Nd:glass laser [175] and from 450 to $720 \mathrm{~nm}$ with $594 \mathrm{~nm}$ pulses from a rhodamine $6 \mathrm{G}$ dye laser [11]. A considerable effort was dedicated to study the SC generation in water with Ti:sapphire laser pulses under various operating conditions [37, 176-179]. These studies demonstrated that a typical SC spectrum covers the 400$1100 \mathrm{~nm}$ range [177]. A broader SC spectrum spanning wavelengths from 350 to $1400 \mathrm{~nm}$ was reported using a thin water jet instead of a thick cell [180]. In the range of anomalous GVD of water (with laser wavelengths above $1 \mu \mathrm{m}$ ), the SC spectrum with the focusing condition-controllable position of the blue peaks was demonstrated when pumped by the fundamental harmonics of the Nd:glass laser (1055 nm) [91]. However, the red shifted portion of the SC spectrum was not measured, as the spectral detection range in these experiments was limited to that of a conventional silicon detector $(1.1 \mu \mathrm{m})$. Although a rapidly increasing infrared absorption and the presence of strong absorption bands at 1.46 and $1.94 \mu \mathrm{m}$, in particular, serve as the main factors that limit the red-shifted broadening of the SC spectrum in water, an unexpectedly broad SC spectrum, ranging from 350 to $1750 \mathrm{~nm}$, was reported using $1.3 \mu \mathrm{m}$ pump pulses from an optical parametric amplifier [181]. A more recent study suggested that under carefully chosen experimental conditions, the absorption effects can be overcome using a thin water jet, thus reducing the nonlinear interaction length just to a few tens of micrometres [182]. Under these settings, with the pump wavelength set at $1.6 \mu \mathrm{m}$, more than two-octave spanning SC spectrum from $300 \mathrm{~nm}$ to $2.4 \mu \mathrm{m}$ was measured.

\subsection{Alkali metal fuorides}

Alkali metal fluorides possess the largest bandgaps among the dielectric materials. Consequently, 
these media exhibit extremely broad transparency windows, which extend from the vacuum ultraviolet to the mid-infrared, making them attractive nonlinear materials for SC generation as pumped with femtosecond laser pulses at various parts of the optical spectrum. In particular, lithium and calcium fluorides, $\mathrm{LiF}$ and $\mathrm{CaF}_{2}$, are the only nonlinear media that are able to produce an appreciable spectral broadening in the deepultraviolet, as demonstrated using the third harmonic pulses from the Ti:sapphire laser [34, 146. When pumped with the second harmonic pulses from the same laser, the measured SC spectra in these media cover the ultraviolet and visible spectral ranges [146, 183, 185.

Extensive experimental studies of $\mathrm{SC}$ generation in alkali metal fluorides were performed with fundamental harmonic pulses from the Ti:sapphire laser, with an emphasis on the blue-shifted part of the SC spectrum. A comparative study of the SC generation in $\mathrm{CaF}_{2}, \mathrm{LiF}$ and $\mathrm{MgF}_{2}$ crystals demonstrated that the $\mathrm{LiF}$ and $\mathrm{CaF}_{2}$ crystals produce the most ultraviolet-shifted supercontinua with the cut-off wavelengths below $300 \mathrm{~nm}$ [186]. Out of these, $\mathrm{CaF}_{2}$ was identified as a promising material for stable SC generation in the ultraviolet and visible spectral ranges, as it exhibits a low induced depolarization [184, 185, 187] and an excellent reproducibility of the spectrum, so important for femtosecond transient absorption spectroscopy [27, 34, 183, 188, 189 and for improving the performance characteristics of UV-pumped noncollinear optical parametric amplifiers [186, 190]. However, $\mathrm{CaF}_{2}$ has a relatively low optical damage threshold, which can be compared to that of fused silica, therefore a reliable and reproducible SC generation in this material is achieved only in the setups, where a continuous translation or rotation of the crystal is performed. Although neglected in some studies [148], the formation of persistent colour centres in LiF was generally considered as a major drawback to its application for SC generation. However, a more recent study revealed that colour centres only slightly modify the ultraviolet cut-off (at $270 \mathrm{~nm}$ ) of the SC spectrum on the long-term (several hours) operation [191].

SC generation in barium fluoride, $\mathrm{BaF}_{2}$, with $800 \mathrm{~nm}$ pumping was studied under various experimental conditions [192, 193]. $\mathrm{BaF}_{2}$ is a well- known scintillator possessing two strong luminescence bands centred at 330 and $200 \mathrm{~nm}$. The former luminescence band was readily employed to map the intensity variation within the light filaments; in particular, a side-view of six-photon absorption-induced luminescence allowed to capture the filament formation dynamics, focusing and refocusing cycles, and eventually to estimate a number of relevant parameters, such as filament diameter, peak intensity, free electron density and multiphoton absorption cross section [136, 194]. From a practical viewpoint, $\mathrm{SC}$ generation in $\mathrm{BaF}_{2}$ was used to provide a broadband seed signal for OPCPA pumped by a high repetition rate Yb:YAG thin disk regenerative amplifier; after amplification the pulses were compressed down to $4.6 \mathrm{fs}$, which is very close to the Fourier limit of the amplified SC spectrum [50].

A more recent study of the SC generation in $\mathrm{CaF}_{2}$ and $\mathrm{BaF}_{2}$ captured the entire $\mathrm{SC}$ spectra, demonstrating a considerable red-shifted broadening, which extends well beyond the detection range of standard Si detectors [162]. More specifically, with $800 \mathrm{~nm}$ pumping, the SC spectra extending from $300 \mathrm{~nm}$ to $2 \mu \mathrm{m}$ and from $320 \mathrm{~nm}$ to $1.98 \mu \mathrm{m}$ were measured in $\mathrm{CaF}_{2}$ and $\mathrm{BaF}_{2}$, respectively, and further red-shifted spectral broadening in these nonlinear crystals was recorded using the pump pulses at $1.38 \mu \mathrm{m}$.

Using the mid-infrared (2.1-2.2 $\mu \mathrm{m})$ pump pulses, whose wavelengths fall into the range of anomalous GVD of the fluoride crystals, combined data from [162] and [195] yield ultrabroad, multioctave SC spectra in $\mathrm{CaF}_{2}$ and $\mathrm{BaF}_{2}$, which span from $340 \mathrm{~nm}$ to $3.3 \mu \mathrm{m}$ and from $350 \mathrm{~nm}$ to $3.8 \mu \mathrm{m}$, respectively. The measurements performed with $15 \mathrm{fs}, 2 \mu \mathrm{m}$ pump pulses revealed an exceptionally flat shape of the SC spectrum in $\mathrm{CaF}_{2}$, showing a broad plateau in the wavelength range of 500-1700 nm [161]. Even broader, almost 4-octave spanning SC spectrum, continuously covering the wavelength range from $290 \mathrm{~nm}$ to $4.3 \mu \mathrm{m}$ (at the $10^{-6}$ intensity level) was reported in LiF with $2.3 \mu \mathrm{m}$ pump pulses [163. Interestingly, such an ultrabroadband spectrum was recorded in the presence of colour centres. The time-resolved evolution of the SC spectra demonstrated that spectral modifications due to the formation of colour centres evolve on a very fast time scale (just a few tens of laser shots). However, after few 
thousands of laser shots the SC spectrum eventually stabilizes and remains unchanged during further operation. Figure 13 compares the SC spectra in a $3.5 \mathrm{~mm}$-long LiF plate, as generated by $100 \mathrm{fs}$ pulses with wavelengths of 1.3 and $2.3 \mu \mathrm{m}$, which fall into the ranges of zero and anomalous GVD of the crystal, respectively, and showing a stable ultraviolet cut-off at $330 \mathrm{~nm}$ (at $10^{-5}$ intensity level).

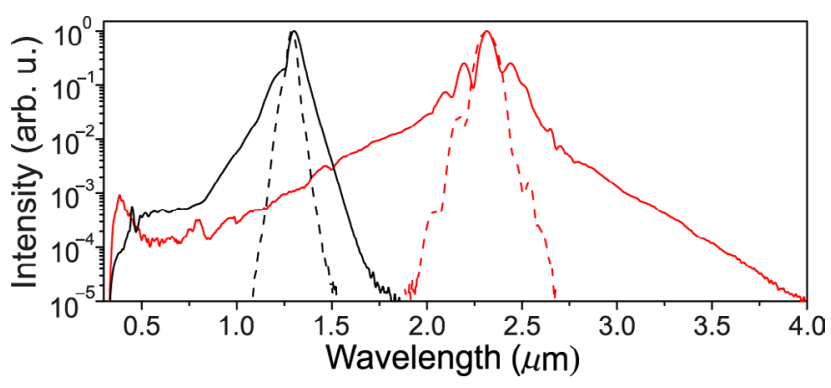

Fig. 13. A comparison of supercontinuum spectra in $3.5 \mathrm{~mm}$-thick $\mathrm{LiF}$ as produced by filamentation of $100 \mathrm{fs}$ pulses with wavelengths of $1.3 \mu \mathrm{m}$ (black curve) and $2.3 \mu \mathrm{m}$ (red (online) curve), which fall into the ranges of zero and anomalous GVD of the crystal and with energies of $2.2 \mu \mathrm{J}$ and $9.5 \mu \mathrm{J}$, respectively. Spectra of the input pulses are shown by dashed curves.

The experiments on SC generation in $\mathrm{CaF}_{2}$ and $\mathrm{BaF}_{2}$ with longer wavelength mid-infrared pulses (tunable in the $2500-3800 \mathrm{~nm}$ range) reported a dramatic change of the SC spectral shape [196, 197. These measurements showed that, for instance, with $3 \mu \mathrm{m}$ pump pulses, the SC spectra are no longer continuous, but are composed of two separate bands, one located in the visible range (400-600 nm) and another in the mid-infrared range $(1500-4500 \mathrm{~nm})$, while the spectral components in the near-infrared spectral range are practically absent or at least their spectral intensities fall below the detection range. Similar results were reported in the LiF crystal, also demonstrating how the spectral position of the detached blue peak (that corresponds to a visible SC band in the above experiments) was shifting from 270 to $500 \mathrm{~nm}$ while changing the input wavelength from 3.3 to $1.9 \mu \mathrm{m}$ [198, 199]. More recent spectral measurements with $3.5 \mu \mathrm{m}$ pulses (Fig. 14), performed over a higher dynamic range, revealed that the spectral discontinuity still exists in $\mathrm{CaF}_{2}$, however, demonstrating a more homogenous $\mathrm{SC}$ spectrum in $\mathrm{BaF}_{2}$, which provided the spec- tral coverage from the visible to beyond $5 \mu \mathrm{m}$ in the mid-infrared range [200].
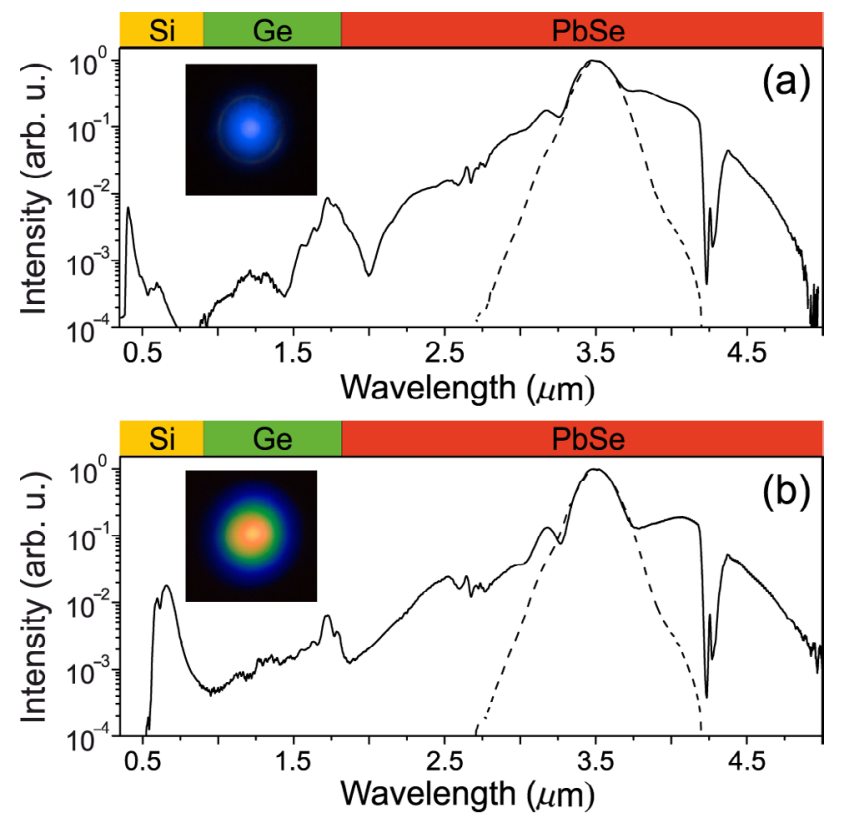

Fig. 14. Supercontinuum spectra generated with $60 \mathrm{fs}$, $31 \mu \mathrm{J}$ pulses at $3.5 \mu \mathrm{m}$ in (a) $\mathrm{CaF}_{2}$ and (b) $\mathrm{BaF}_{2}$, both of $4 \mathrm{~mm}$ thickness. Dashed curves show the input pulse spectrum. A deep double dip around $4.25 \mu \mathrm{m}$ is due to the absorption of atmospheric $\mathrm{CO}_{2}$. The ranges of spectrometer detectors $(\mathrm{Si}, \mathrm{Ge}, \mathrm{PbSe})$ are indicated by colour (online) bars on the top. The insets show the visual appearances of the SC beams in the far field. Adapted from [200].

\subsection{Laser hosts}

Laser host crystals, such as undoped sapphire $\left(\mathrm{Al}_{2} \mathrm{O}_{3}\right)$ and yttrium aluminum garnet (YAG), are excellent nonlinear media possessing a good crystalline quality, a high nonlinearity and a high optical damage threshold. Therefore it is quite surprising that the potential of these laser host materials for SC generation was systematically overlooked in the early studies.

The first experimental demonstration of the SC generation in sapphire dates back to 1994 [201], revealing sapphire as a long sought solid-state material to replace the liquid media commonly used at that time, putting the technology of femtosecond optical parametric amplifiers on all-solid state grounds (see also [202] for more details). Since then, sapphire became a routinely used nonlinear medium for SC generation with Ti:sapphire 
driving lasers, providing a high quality seed signal that boosted the development of modern ultrafast optical parametric amplifiers [45, 47, 203].

Pumping the sapphire crystal with the second harmonics of the Ti:sapphire laser $(400 \mathrm{~nm})$, the SC spectrum in the $350-700 \mathrm{~nm}$ range was produced (please refer to Fig. 20(a), which appears in Section 7 of the paper). In the visible spectral range, using the second harmonics of an amplified Yb-fibre laser $(515 \mathrm{~nm})$ as a pump, the SC spectrum from 340 to $650 \mathrm{~nm}$ was measured, which was thereafter used to seed the noncollinear optical parametric amplifier pumped by the third harmonic $(343 \mathrm{~nm})$ of the same laser, rendering an unprecedented tuning in the near-ultraviolet and blue spectral ranges [204]. A typical SC spectrum in sapphire covers the wavelength range from 410 to $1100 \mathrm{~nm}$, as generated with fundamental harmonic pulses from the Ti:sapphire laser (pump wavelengths of around $800 \mathrm{~nm}$ ) and measured under the commonly used operating conditions (input pulse energy of $\sim 1 \mu \mathrm{J}$ and material thickness of 1-3 mm) [26, 28, 30, 205]. A notable extension of the infrared part of the SC spectrum was demonstrated by employing looser focusing geometry and somewhat longer sapphire samples; under these focusing conditions an appreciable red-shifted SC signal extended to more than $1600 \mathrm{~nm}$ [26, 133] (see Fig. 15(a)). A broader, spectrally flat SC was produced using pump wavelengths around the zero GVD point of sapphire $(1.31 \mu \mathrm{m})$ [206]. Such SC was used to seed the OPCPA system driven by diode-pumped $\mathrm{Yb}: \mathrm{KGW}$ and Nd:YAG lasers, finally producing carrier envelope phasestable sub-9 fs pulses with $5.5 \mathrm{TW}$ peak power at $1 \mathrm{kHz}$ repetition rate [52]. In the range of anomalous GVD of sapphire, the SC spectrum spanning wavelengths from $470 \mathrm{~nm}$ to more than $2.5 \mu \mathrm{m}$ were reported with $15 \mathrm{fs}$ pulses at $2 \mu \mathrm{m}$, however, showing a noticeable drop of the spectral intensity around $1 \mu \mathrm{m}$ [161].

Unlike many other laser host crystals, YAG has no birefringence due to its cubic structure. This property, combined with an excellent crystalline quality, a high nonlinearity and a high optical damage threshold, makes YAG a very attractive nonlinear medium for SC generation. When pumped in the visible spectral range $(515 \mathrm{~nm})$, YAG produces a narrower (390-625 nm) and more structured SC spectrum than that obtained in sap-

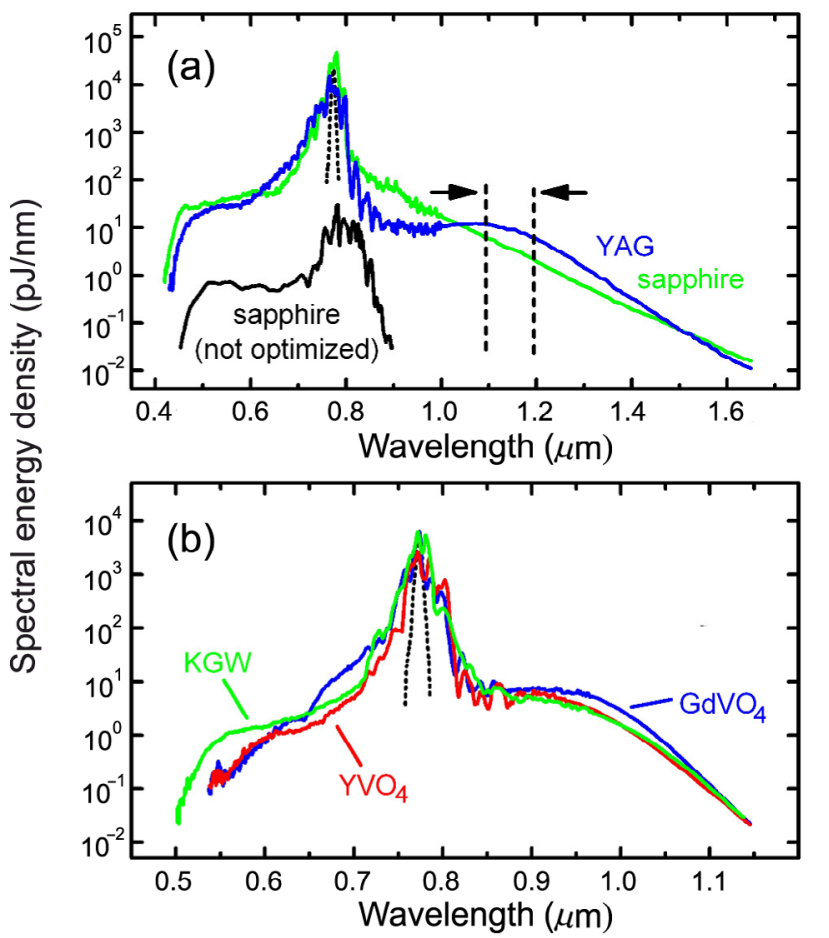

Fig. 15. (a) Black curve: supercontinuum spectrum from $3 \mathrm{~mm}$ sapphire with conventional (tight focusing) pumping conditions (shown not to scale, shifted for clarity). Green (online) curve: supercontinuum spectrum from a $3 \mathrm{~mm}$ sapphire with the loose focusing condition. Blue (online) curve: supercontinuum spectrum from $4 \mathrm{~mm}$ YAG showing an improved photon density in the infrared region. (b) Supercontinuum spectra generated in $\mathrm{KGW}$ (green (online) curve), $\mathrm{YVO}_{4}$ (red (online) curve) and $\mathrm{GdVO}_{4}$ (blue (online) curve) crystals (all $4 \mathrm{~mm}$ ) with the input pulse energies of 83, 59 and $78 \mathrm{~nJ}$, respectively. Adapted from [26].

phire [204]. However, the advantages of the YAG crystal show up with longer pump wavelengths. With near-infrared pump wavelengths, YAG produces a quite similar SC spectrum as sapphire (420-1600 nm, with $800 \mathrm{~nm}$ pumping), which exhibits a higher spectral intensity in the infrared, as illustrated in Fig. 15(a), and which is obtained with reduced, sub- $\mu \mathrm{J}$ pump energies due to a large nonlinear index of refraction of the crystal [26]. The SC generation in YAG was also studied with 1.1-1.6 $\mu \mathrm{m}$ tunable pulses from an optical parametric amplifier, demonstrating a fairly stable blue cut-off at $530 \mathrm{~nm}$ and a progressive extension of the infrared part of the spectrum while increasing the pump wavelength [26].

With the mid-infrared pumping, in the range of anomalous GVD (for input wavelengths longer 
than $1.6 \mu \mathrm{m}$ ), YAG shows an advantage over sapphire in producing a much flatter SC spectrum over the entire wavelength range. SC generation with a spectrum extending from $510 \mathrm{~nm}$ to more than $2.5 \mu \mathrm{m}$ was reported with carrier envelope phase-stable $15 \mathrm{fs}$ pulses at $2 \mu \mathrm{m}$ [161] and from $450 \mathrm{~nm}$ to more than $2.5 \mu \mathrm{m}$ with 32 fs pulses at $2.15 \mu \mathrm{m}$ [207], both demonstrating preservation of a stable carrier envelope phase of the broadband radiation. However, in these experiments the longest detectable wavelength was limited to $2.5 \mu \mathrm{m}$. The full spectral extent of SC was measured more recently, reporting the generation of almost four optical octave spanning SC with a continuous wavelength coverage from $350 \mathrm{~nm}$ to $3.8 \mu \mathrm{m}$, as demonstrated using $100 \mathrm{fs}, 2.3 \mu \mathrm{m}$ pump pulses from an optical parametric amplifier [163]. With even longer, 3.1 $\mu \mathrm{m}$ pump pulses delivered by a high repetition rate OPCPA system, the multioctave (from $450 \mathrm{~nm}$ to more than $4.5 \mu \mathrm{m}$, Fig. 16), carrier envelope phase-stable SC was reported, potentially yielding a single optical cycle self-compressed pulses at the output of the crystal, as predicted by numerical simulations [80].

The high optical damage threshold of sapphire and YAG, in particular, identifies them as suitable nonlinear materials for SC generation with longer laser pulses, of durations varying from

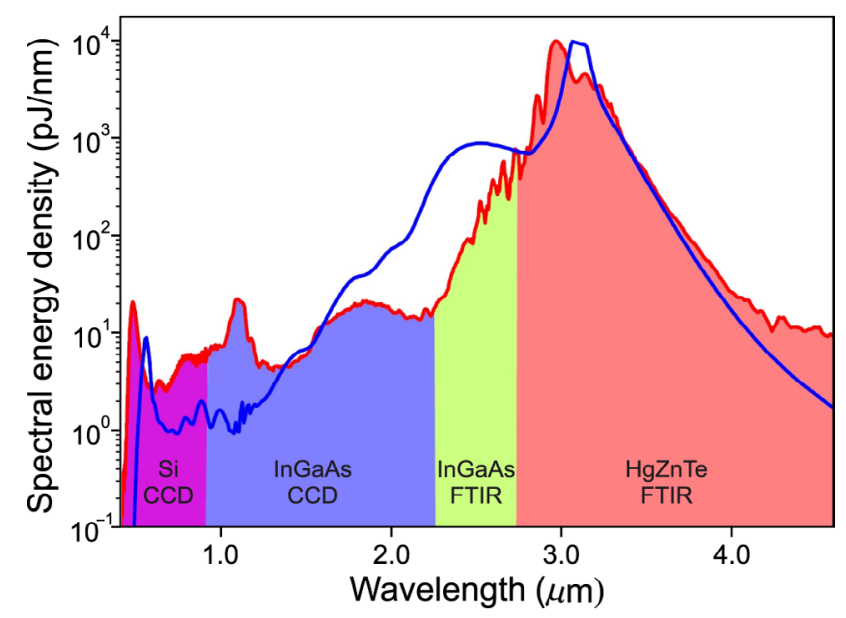

Fig. 16. Supercontinuum spectrum generated by $3.1 \mu \mathrm{m}, 2.6 \mu \mathrm{J}$ pulses in a $2 \mathrm{~mm}$-thick YAG plate (red (online) curve). The ranges of each spectrometer/detector ( $\mathrm{Si}$, InGaAs and $\mathrm{HgCdZnTe}$ ) are indicated by colour (online) fills. Superimposed is the angle-integrated spectrum from the numerical simulation (blue (online) curve). Adapted from [80]. a few hundreds of femtoseconds to a few picoseconds. The renewed interest in SC generation in solid state dielectric media with picosecond laser pulses was prompted by the development of novel Yb-based solid-state lasers operating around $1 \mu \mathrm{m}$ and dedicated to the development of compact, efficient, and inexpensive tabletop OPCPA systems. In this regard, picosecond SC is expected to provide a robust broadband seeding source, which would markedly simplify the OPCPA architecture, excluding the need for an optically or electronically synchronized broadband laser oscillator source.

The first studies aiming at stable SC generation were performed with Ti:sapphire laser pulses of variable duration, as accessed via either spectral narrowing or pulse chirping [208, 209]. These investigations were followed-up by using genuine sub-picosecond pulses from Yb:KYW [210] and 1-ps pulses from Nd:glass [211, 212] lasers, demonstrating that under carefully chosen experimental conditions a stable and reproducible SC is generated without damaging the nonlinear material. Experimental and numerical investigations have revealed that the filamentation of picosecond laser pulses forms a more complex spatiotemporal structure of SC, which is governed by free electron plasma, in contrast to femtosecond filamentation dynamics, which is mainly driven by multiphoton absorption [212]. However, the spectral-temporal analysis has shown that the red-shifted and blue-shifted spectral broadenings are associated just with two distinct sub-pulses, which are well separated in time. The high temporal coherence of sub-picosecond pulse-generated SC was directly verified by the spectral phase measurements of parametrically amplified SC pulses [210]. Indeed, the availability of picosecond pulse-generated SC with a well-behaved spectral phase that is compressible down to transform-limit paved new avenues in the development of picosecond-laser pumped noncollinear optical parametric amplifiers [213] and of a whole new generation of compact SC-seeded OPCPA systems. These systems are built around amplified solely sub-picosecond and picosecond lasers, such as Yb:KYW 214, Yb:YAG [51, 207, 215-217] and Yb-fibre [218, 219], and are designed to provide few optical cycle pulses in the near- and mid-infrared at very high repetition rates. Finally, picosecond SC serves as a seed signal in the mid-infrared optical 
parametric amplifiers driven by amplified picosecond Ho:YAG lasers [220, 221].

The SC generation was also studied in other laser host materials, such as potassium gadolinium tungstate $(\mathrm{KGW})$, gadolinium vanadate $\left(\mathrm{GdVO}_{4}\right)$ and yttrium vanadate $\left(\mathrm{YVO}_{4}\right)$ using Ti:sapphire laser pulses [26]. These crystals exhibit high nonlinear refractive indexes, which result in a very low critical power for self-focusing and allow SC generation with sub-100 nJ input pulse energies. However, due to relatively small bandgaps and transmission cut-off wavelengths located in the near-ultraviolet, the measured spectral broadening in these materials was rather modest and covered the spectral ranges of $500-1150 \mathrm{~nm}$ in $\mathrm{KGW}$, and 550-1150 $\mathrm{nm}$ in $\mathrm{GdVO}_{4}$ and $\mathrm{YVO}_{4}$ crystals (see Fig. 15(b)). SC generation with 800$1500 \mathrm{~nm}$ tunable pump pulses was also studied in gadolinium orthosilicate (GSO), gallium gadolinium garnet (GGG), lithium tantalate (LTO) and lutetium vanadate (LVO) crystals [222]. The authors measured just the anti-Stokes (blue-shifted) portions of the SC spectra and recoded fairly constant cut-off wavelengths of $450 \mathrm{~nm}$ in GSO and GGG, and 550 and $650 \mathrm{~nm}$ in LTO and LVO crystals, respectively.

\subsection{Birefringent crystals}

Noncentrosymmetric nonlinear crystals which exhibit birefringence and possess second-order nonlinearity are indispensable nonlinear media serving for laser wavelength conversion via nonresonant three-wave interactions, such as the second harmonic, sum and difference frequency generation, and optical parametric amplification. The joint contribution of quadratic and cubic nonlinearities adds unique features to the SC generation process in these crystals.

The most simple and straightforward approach of SC generation in noncentrosymmetric crystals neglects the second-order nonlinear effects. To date, filamentation and SC generation in the potassium dihydrogen phosphate (KDP) crystal by launching the pump beam along the optical axis of the crystal was investigated experimentally [223225] and numerically [226]. The SC generation was also reported in the absence of phase matching in lithium triborate (LBO) [89] and lithium niobate (LN) [227] crystals, and in the $\alpha$-barium borate
$(\alpha$-BBO) crystal, which exhibits birefringence, but vanishing second-order nonlinearity [228].

Filamentation under the conditions of phasematched second-order nonlinear effects has led to SC generation, which is accompanied by simultaneous wavelength-tunable second harmonic or sum frequency generation, as experimentally observed in basic nonlinear crystals, such as KDP [229, 230] and $\beta$-barium borate $(\beta$-BBO) [231, 232].

A unique aspect of self-action phenomena in birefringent media, possessing both quadratic and cubic nonlinearities, which was generally neglected in the above studies, is the so-called secondorder cascading. The cascading effect arises from the phase-mismatched second-harmonic generation, which leads to a recurrent energy exchange between the fundamental and second harmonic frequencies, imprinting large nonlinear phase shifts on the interacting waves [233]. The cascading effect mimics the Kerr-like behaviour arising from the intrinsic cubic nonlinearity and hence produces a large cascaded nonlinear index of refraction, whose sign and magnitude could be easily varied by rotating the crystal in the phase-matching plane or changing its temperature (Fig. 17) and which is an important asset to the nonlinear dynamics of femtosecond pulses [234, 235].

There are two different ways how the secondorder cascading may be favourably exploited for generation of broadband spectra in bulk nonlinear

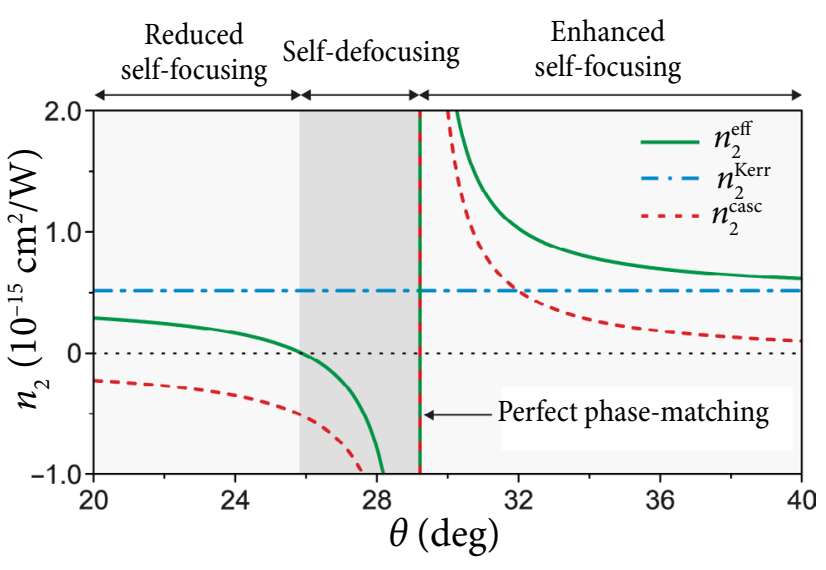

Fig. 17. Nonlinear refractive indices of BBO crystal at $800 \mathrm{~nm}$ : intrinsic, $n_{2}^{\text {Kerr }}$ (blue (online) dash-dotted line), cascaded, $n_{2}^{\text {casc }}$ (red (online) dashed curve) and effective, $n_{2}^{\text {eff }}=n_{2}^{\text {Kerr }}+n_{2}^{\text {casc }}$ (green (online) solid curve) versus the angle $\theta$, which is the angle between the propagation direction and the optical axis of the crystal. 
crystals. The first approach makes use of the selfdefocusing propagation regime, where the cascaded nonlinear index of refraction, $n_{2}^{\text {casc }}$, is negative and its absolute value is greater than the competing intrinsic $n_{2}^{\text {Kerr }}$, so $n_{2}^{\text {eff }}<0$. Under these operating conditions, spectral broadening is achieved without the onset of filamentation and results from pulse self-compression and temporal soliton generation, which in turn emerges from the opposite action of self-phase modulation and material dispersion [236].

The numerical simulations predicted that the soliton compression-induced SC generation regime of the soliton could be achieved in a variety of nonlinear crystals, which possess the normal GVD for the pump wavelength [237]. More recent numerical and experimental results show that the long wave side of the $\mathrm{SC}$ radiation could be enriched by the generation of broadband dispersive waves located beyond the zero dispersion wavelength of the crystals: together with the soliton the dispersive waves contribute to the octave spanning SC, which extends from 1.0 to $4.0 \mu \mathrm{m}$ in $\mathrm{LN}$ [238] and from 0.9 to $2.3 \mu \mathrm{m}$ in $\beta$-BBO [239] crystals. More recently, this approach was extended to the nonlinear crystals that are transparent in the mid-infrared range [240]. To this end, the SC covering wavelength range from 1.6 to $7.0 \mu \mathrm{m}$ was experimentally measured in the lithium thioindate $\left(\mathrm{LiInS}_{2}\right)$ crystal, using the pump pulses in the $3-4 \mu \mathrm{m}$ range.

The soliton compression and SC generation in the self-defocusing regime is particularly attractive concerning the energy scaling, since a favourable interplay between the self-phase modulation and material dispersion is achieved without the onset of beam filamentation.

In contrast, the second approach makes use of the self-focusing propagation regime, which leads to filamentation. Here the cascaded nonlinearity could be used either to enhance or reduce the effective nonlinear index of refraction, $n_{2}^{\text {eff }}$ (see Fig. 17), hence opening the possibility to perform the nonlinear interaction in a controlled way. To this end, the interplay between the cascadedquadratic and intrinsic cubic nonlinearities was favourably exploited to achieve filamentation and SC generation with nearly monochromatic, 30 ps pulses from the Nd:YAG laser in the periodically poled LN crystal, also demonstrating the control of the SC spectral extent by tuning the temperature [241]. In the femtosecond regime, the filamentation and SC generation in the $\beta$-BBO crystal with the input wavelengths falling in the range of normal [242] and anomalous [86] GVD was reported. In the regime of normal GVD, SC with the spectral extent from $410 \mathrm{~nm}$ to $1.1 \mu \mathrm{m}$ was generated with Ti:sapphire laser pulses at $800 \mathrm{~nm}$, demonstrating control of the blue-shifted portion of the SC spectrum by tuning the angle between the incident laser beam and the optical axis of the crystal [242] (see Fig. 18). The achieved spectral control was very robust in terms of input pulse energy and was attributed to an efficient generation of the self-phase-matched second harmonic, which introduced a considerable energy loss and distortion of the trailing sub-pulse shape, counteracting the joint effect of cascadedquadratic and cubic self-steepenings. A broader

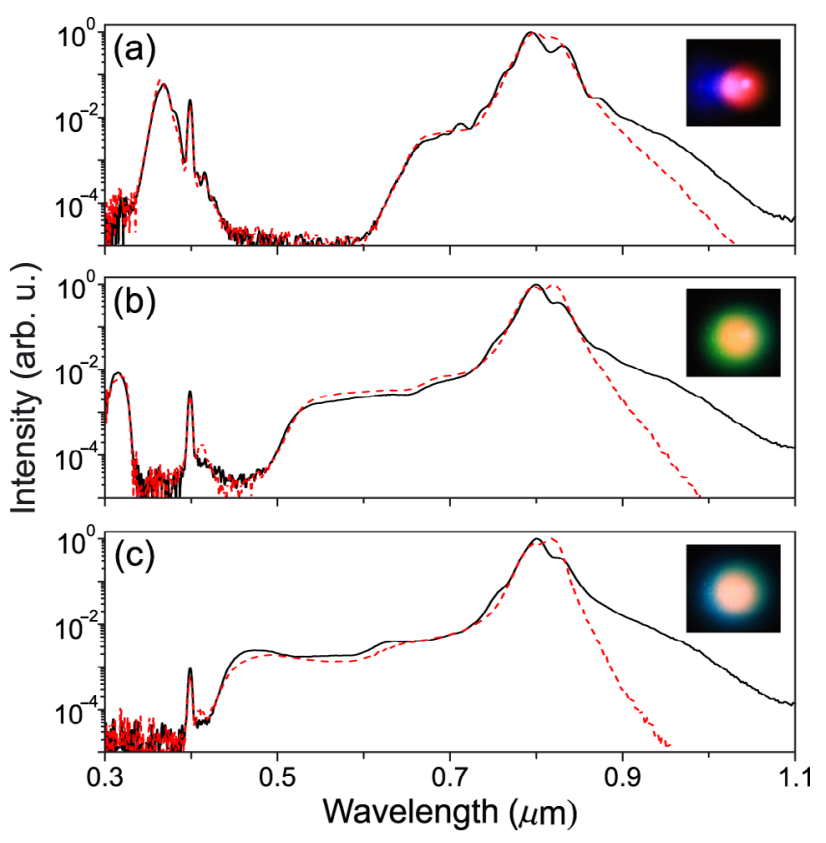

Fig. 18. Supercontinuum spectra in a $5 \mathrm{~mm}$-thick BBO crystal as generated with $100 \mathrm{fs}, 290 \mathrm{~nJ}$ input pulses at $800 \mathrm{~nm}$ (black solid curves) and measured at various crystal detunings from the perfect phase matching angle $\left(29.2^{\circ}\right.$ ): (a) $32.4^{\circ}$, (b) $37.5^{\circ}$, c) $42.6^{\circ}$. A narrow peak at $400 \mathrm{~nm}$ and a broad peak with a tunable wavelength are the phase-mismatched and self-phase-matched second harmonics. The insets show the visual appearances of the SC beam in the far field. Red (online) dashed curves show the supercontinuum spectra generated with lower (190 nJ) input pulse energy. Adapted from the data of [242]. 
SC spectrum, extending from $520 \mathrm{~nm}$ to $2.5 \mu \mathrm{m}$, was generated with $1.8 \mu \mathrm{m}$ pulses from an optical parametric amplifier, whose wavelength falls into the anomalous GVD range of the $\beta$-BBO crystal [86]. Here the ultrabroadband SC spectrum was generated due to the formation of spatiotemporal light bullets, which experience more than 4 -fold self-compression from 90 down to 20 fs. Moreover, in the range of enhanced self-focusing (where $n_{2}^{\text {casc }}$ is positive), a markedly reduced filamentation threshold was experimentally detected, that led to SC generation at sub-critical powers for self-focusing.

The second-order cascading-mediated spectral broadening was also reported under different experimental settings. Generation of intense SC with a spectrum between 1.2 and $3.5 \mu \mathrm{m}$ and with sub-mJ energy input pulses at $1.5 \mu \mathrm{m}$ was demonstrated in a highly nonlinear organic DAST crystal [243. A dramatic spectral broadening of subtwo optical cycle pulses with a central wavelength of $790 \mathrm{~nm}$ was achieved in the $\beta$-BBO crystal due to multi-step cascaded interactions yielding an ultrabroadband spectrum in the $0.5-2.4 \mu \mathrm{m}$ range [244].

\section{Supercontinuum generation in semiconductors}

Bulk semiconductors possess much larger cubic nonlinearities than dielectrics and extended transparency windows in the mid-infrared spectral range, and so emerge as promising nonlinear media for hosting nonlinear interactions in the near- and mid-infrared ranges [245]. The midinfrared spectral range is particularly interesting for spectroscopic studies since it covers the socalled molecular fingerprint region. The feasibility of semiconductor materials for SC generation in the mid-infrared spectral range was experimentally demonstrated more than 30 years ago [246]. Here the authors studied spectral broadening in gallium arsenide ( $\mathrm{GaAs})$, zinc selenide $(\mathrm{ZnSe})$, and cadmium sulphide (CdS) crystals with picosecond pulses at $9.3 \mu \mathrm{m}$ from an amplified $\mathrm{CO}_{2}$ laser. The broadest SC spectrum, which spanned from 3 to $14 \mu \mathrm{m}$, was measured in the GaAs crystal.

From the time perspective, the real progress in the field was achieved only quite recently. $2-20 \mu \mathrm{m}$ spanning picosecond SC in GaAs was reported using $2.5 \mathrm{ps}, 9.3 \mu \mathrm{m}$ pulses from a $\mathrm{CO}_{2}$ laser [247]. In the femtosecond regime, output spectrum spanning wavelengths from 3.5 to $7 \mu \mathrm{m}$ was generated in a $10-\mathrm{mm}$ thick GaAs crystal when pumped by $100 \mathrm{fs}$ pulses with a central wavelength of $5 \mu \mathrm{m}$, as obtained by the difference frequency generation between the signal and idler pulses of the Ti:sapphire laser-pumped near-infrared optical parametric amplifier [248]. More recently, a GaAs crystal was used in a similar setup to generate SC in the $4-9 \mu \mathrm{m}$ range, using $4.2-6.8 \mu \mathrm{m}$ tunable pulses. The authors also reported pulse compression down to sub-two optical cycle widths around $6 \mu \mathrm{m}$ by employing the external post compression of spectrally broadened pulses in $\mathrm{BaF}_{2}$, $\mathrm{CaF}_{2}$ and $\mathrm{MgF}_{2}$ crystals, which possess anomalous GVD [249]. 3-18 $\mu \mathrm{m}$ spanning SC was generated in the GaAs crystal when pumped by femtosecond laser pulses with a central wavelength of $7.9 \mu \mathrm{m}$, which falls into the range of anomalous GVD of GaAs [250]. Along with spectral superbroadening, a simultaneous pulse self-compression down to almost a single optical cycle was measured. More than three octave-wide supercontinuum, with the spectrum from $500 \mathrm{~nm}$ to $4.5 \mu \mathrm{m}$, was generated in a zinc sulphide ( $\mathrm{ZnS}$ ) crystal pumped with 27 fs pulses at $2.1 \mu \mathrm{m}$ from the OPCPA system [195].

A series of interesting results on spectral broadening and SC generation have been recently reported in a $\mathrm{ZnSe}$ crystal. Filamentation and SC generation was studied in the ZnSe crystal using pump pulses with a tunable wavelength and so accessing self-focusing regimes of different orders of multiphoton absorption [251]. However, no filamentation was observed with $800 \mathrm{~nm}$ pump pulses, while only a very modest spectral broadening in the near-infrared spectral range was achieved with a pump wavelength at $1.2 \mu \mathrm{m}$. The potential of the ZnSe crystal to produce an ultrabroadband SC for spectroscopic applications was demonstrated using the pump pulses with a central wavelength of $5 \mu \mathrm{m}$, that is close to the zero GVD wavelength $(4.8 \mu \mathrm{m})$ of the crystal. SC spectrum spanning wavelengths from $500 \mathrm{~nm}$ to $11 \mu \mathrm{m}$ was measured in the multifilamentation regime [252]. In addition to its high cubic nonlinearity, $\mathrm{ZnSe}$ owes an attractive set of optical properties, such as a polycrystalline structure and a non-vanishing 
second-order nonlinearity, which produce a number of interesting nonlinear effects, enriching the spectral content of SC. Spectral broadening around the carrier wavelength, accompanied by a simultaneous generation of multiple broadband even and odd harmonics, was reported in polycrytalline $\mathrm{ZnSe}$ samples using few optical cycle pulses at $3 \mu \mathrm{m}$ [219], and at $3.5 \mu \mathrm{m}$ and $5.2 \mu \mathrm{m}$ [220] from recently developed mid-infrared OPCPA systems. Figure 19(a) shows a photograph of a light filament in a $5 \mathrm{~mm}$-long $\mathrm{ZnSe}$ sample, excited by $100 \mathrm{fs}, 2.4 \mu \mathrm{m}$ pulses from an optical parametric amplifier. An ultrabroadband SC spectrum covering the wavelength range from $600 \mathrm{~nm}$ to $4.2 \mu \mathrm{m}$, that corresponds to 2.8
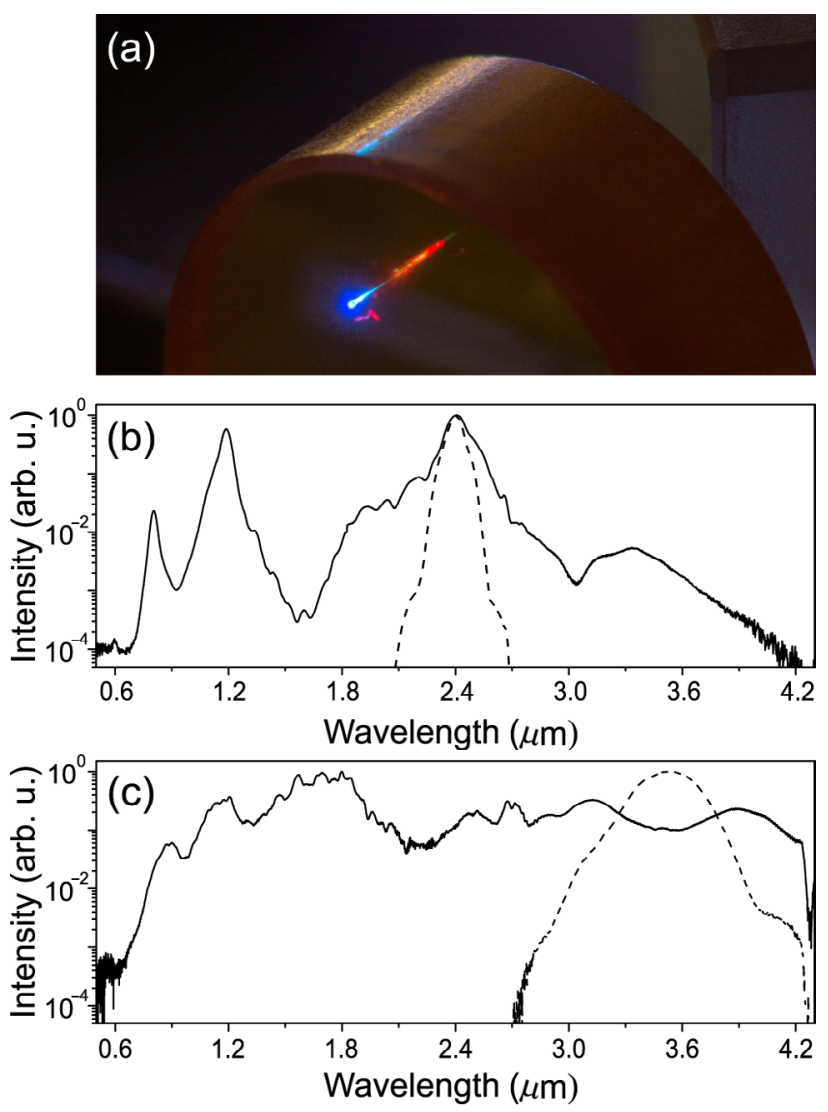

Fig. 19. (a) Photograph of a light filament in ZnSe excited by $100 \mathrm{fs}, 2.4 \mu \mathrm{m}$ pulses: the blue (online) track is the plasma luminescence, the red (online) track is the visible portion of spectrally broadened third harmonic at $800 \mathrm{~nm}$. Supercontinuum spectra in a $5 \mathrm{~mm}$-thick polycrystalline ZnSe plate, as generated by filamentation of: (b) $100 \mathrm{fs}, 3 \mu \mathrm{J}$ pulses at $2.4 \mu \mathrm{m}$, adapted from [253]; (c) $60 \mathrm{fs}, 6.5 \mu \mathrm{J}$ pulses at $3.5 \mu \mathrm{m}$. A sharp drop of the spectral intensity at $4.25 \mu \mathrm{m}$ is due to $\mathrm{CO}_{2}$ absorption. The input pulse spectra are shown by dashed curves. optical octaves [253], is illustrated in Fig. 19(b). Prominent broadband spectral peaks at $1.2 \mu \mathrm{m}$ and $800 \mathrm{~nm}$ are associated with the second and third harmonics, respectively, which were simultaneously generated by three-wave mixing processes. A very efficient harmonics generation was attributed to a randomly quasi phase-matched broadband three-wave mixing, which stems from the disorder of the nonlinear domains (randomly oriented individual crystallites) [254]. Self-focusing and filamentation of longer-wavelength $(3.5 \mu \mathrm{m})$ input pulses, generated by difference frequency mixing between the signal and idler waves of the optical parametric amplifier, produced a reasonably flat, plateau-like SC spectrum, which results from merging of the spectral broadening around the incident wavelength and the second, third and fourth harmonics peaks, extending beyond the long-wave detection limit of the spectrometer, as shown in Fig. 19 (c).

\section{Control of supercontinuum generation}

The rapidly expanding field of applications calls for achieving broadband radiation with desired temporal and spectral properties, which in turn require setting an efficient control on the filamentation process. As described in the previous sections, the filamentation dynamics and hence the spectral content of the SC are defined essentially by the laser wavelength and by linear and nonlinear properties of the medium, such as material dispersion, bandgap and the nonlinear index of refraction [15, 61]. These material parameters possess fundamental mutual relationships [59] and therefore are generally fixed for a given nonlinear medium at a given input wavelength, so reducing the possibilities to influence the filamentation and SC generation dynamics in real experimental settings.

Several conceptually different approaches have been proposed to overcome these limitations, allowing to modify the spectral content of the SC radiation in a controlled fashion. The first approach relies on tailoring and shaping the parameters of the input laser beam and pulse. To this end, a certain control of filamentation and SC generation processes was achieved by varying the polarization state (from linear to circular) of the incident radiation in isotropic media [255, 256] and by 
choosing either ordinary or extraordinary input pulse polarizations in birefringent media with zero second-order susceptibility, e.g. $\alpha$-BBO crystal [228]. Phase and amplitude shaping of the input pulse allowed a significant (by the order of magnitude) increase of the spectral intensity of the SC within specified bandwidths [257]. More recently, tailoring the input pulse shape by introducing second- and third-order phase distortions, which were controlled by an acousto-optic programmable dispersive filter, was demonstrated to affect the pulse splitting dynamics, which in turn resulted in generation of the SC with a controllable spectral bandwidth and shape [258]. A similar approach based on employing a 4 -f pulse shaper with a phase mask produced by a liquid crystal spatial light modulator was demonstrated for achieving accumulation of the spectral intensity at selected frequencies by a controllable amount [259].

Setting of either positive or negative chirp [260] or adjustment of the focal plane of the input pulses [91] allowed a controllable tuning of the blueshifted cut-off of the SC spectrum. On the other hand, enhancement of the red-shifted SC spectral extent was achieved by setting a low numerical aperture of the input beam and using a longer nonlinear medium [26, 133]. A notable extension of the SC spectrum and enhancement of the SC components within certain spectral ranges, in particular, was achieved by means of two colour filamentation, i.e. by launching two filamentforming beams of different wavelengths [261]. An additional degree of freedom in controlling the SC generation process via the time delay between the input pulses was demonstrated using crossing beams in the single [262] and to some extent in the multiple [263] filamentation regimes. Figure 20(a) shows the individual SC spectra in sapphire, generated by the filamentation of separately launched $300 \mathrm{fs}$ pulses at fundamental $(1030 \mathrm{~nm})$ and second harmonic $(515 \mathrm{~nm})$ wavelengths from an amplified Yb:KGW laser. Figure 20(b) shows an example how the spectral intensity within certain spectral regions and the overall shape of the resulting SC spectrum is modified by varying the time delay between the co-filamenting fundamental and second harmonic pulses. An efficient control of the SC spectrum was also performed by changing the relative position of the focus in the nonlinear medium by means of diffractive
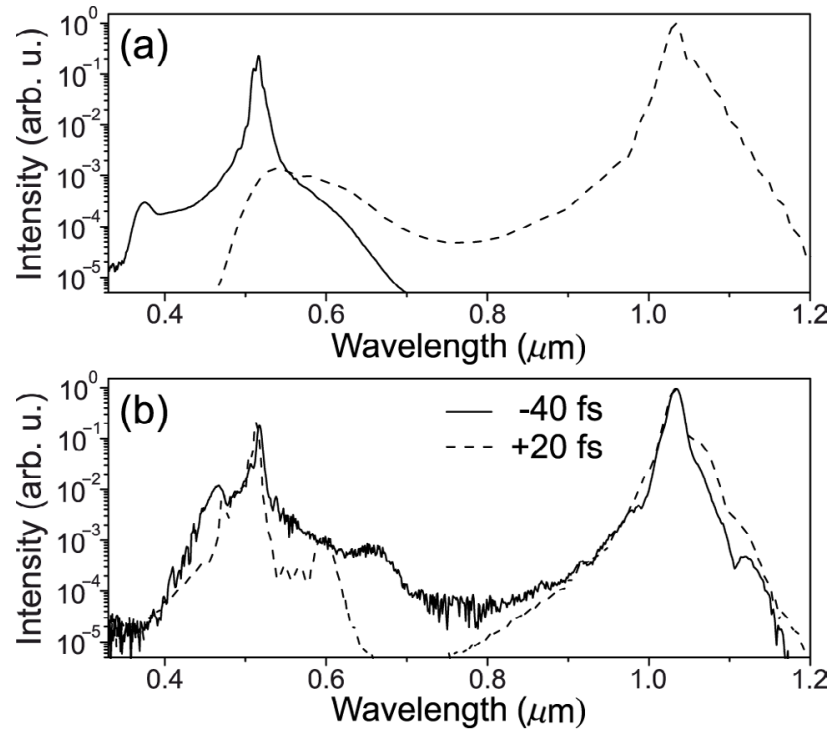

Fig. 20. (a) Individual supercontinuum spectra generated in a $3 \mathrm{~mm}$-long sapphire plate by filamentation of $300 \mathrm{fs}$ pulses at $1030 \mathrm{~nm}$ and $515 \mathrm{~nm}$, each containing a power equal to $2.6 P_{\mathrm{cr}^{\circ}}$ (b) Resulting SC spectra generated by co-filamentation of the above pulses. The negative delay time indicates that the second harmonic pulse comes first. Courtesy of M. Vengris.

optics [264, 265] or by varying the spatial phase of the input beam with spatial light modulators [266, 267]. Fine adjustment of the position of the nonlinear focus was demonstrated by varying the carrier envelope phase of a few-cycle input pulse [268].

The second approach is based on tailoring the linear (absorption) and nonlinear properties (nonlinear index of refraction) of the medium itself. As suggested in the pioneering work [269], various dopants may lead to an enhanced nonlinear optical response of the medium and thus lowering the threshold condition for nonlinear processes, which in turn result in a modification of the SC spectral shape. At present, these early ideas received further attention by demonstrating SC generation in silver [270] and gold [271] nanoparticles- and gold nanorods [181]-doped water and in the LN crystal doped with $\mathrm{Ni}$ ions [227]. Femtosecond SC generation was also reported in more complex nonlinear media, such as aqueous colloids containing silver nanoparticles [272] and nanocomposite materials [273]. Filamentation of femtosecond laser pulses in aqueous solutions with absorbing inorganic [274] or protein [275] dopants was shown to produce a more spectrally 
flat SC. In contrast, narrowed and discontinuous SC spectra were generated in laser-modified nonlinear solid-state media, such as fused silica [276] and YAG [84]. Very promising results regarding an efficient control of the SC spectrum were obtained by making the use of the second-order cascading effects in birefringent crystals, as discussed in more detail in Subsection 5.5 of the paper.

\section{Power scaling and applications to pulse compression}

Emerging applications in many fields of ultrafast science and extreme nonlinear optics require a high power broadband radiation and few optical cycle pulses that are generated by all-solid-state technology and by relatively simple means. Pulse compression based on increasing the spectral bandwidth via self-phase modulation in a bulk solid-state medium and a subsequent removal of the frequency modulation by using an appropriate dispersive delay line represents a simple and universal method for obtaining few optical cycle pulses [277, 278].

Recently, a flexible and versatile approach for the generation of high power, high energy SC in a solid-state medium was proposed and demonstrated by using a series of distributed thin plates instead of a single piece of a bulk nonlinear medium [279]. The individual fused silica plates were located so that the optical pulse exits each plate before the onset of small scale self-focusing and break-up of a high power beam into multiple filaments, yielding high energy broadband radiation with a uniform spatial profile. The proposed approach was further elaborated by demonstrating the scaling of the input peak power as much as two thousand times the critical power for self-focusing in the solid-state medium 280. Remarkably, the cascaded spectral broadening in a distributed multi-plate arrangement was demonstrated to yield the SC pulse with a regular chirp, which is compressible to a nearly transform-limit using an external pulse compressor [279], and this approach could be easily implemented in many schemes for achieving few optical cycle pulses. Indeed, $250 \mathrm{fs}$ output pulses from a Yb:YAG thin disc oscillator were compressed down to 15 fs [281]. More recently, the input $0.8 \mathrm{~mJ}, 30 \mathrm{fs}$ laser pulses at $790 \mathrm{~nm}$ were spectrally broadened in a sequence of 7 fused silica plates of $100 \mu \mathrm{m}$ thickness each and compressed using chirped mirrors down to 5.4 fs (two optical cycles) with almost $90 \%$ throughput efficiency [282]. A multi-plate approach was also used to generate a high power broadband SC in a sequence of 7 alternating UV-grade fused silica and sapphire plates of $200 \mu \mathrm{m}$ thickness (Fig. 21(a)) with a tunable pump wavelength from 1.1 to $1.75 \mu \mathrm{m}$, which covers the regimes of normal, zero and anomalous GVD of fused silica and sapphire and with pump energy up to $0.5 \mathrm{~mJ}$ [283]. Figures 21 (b, c) show the SC spectra as functions of the plate number recorded with the input pulse wavelengths of 1.3 and $1.6 \mu \mathrm{m}$, respectively. A comparison between the SC generated in multiple plates and a continuous nonlinear medium showed that the energy scaling is achieved without the degradation of

(a)
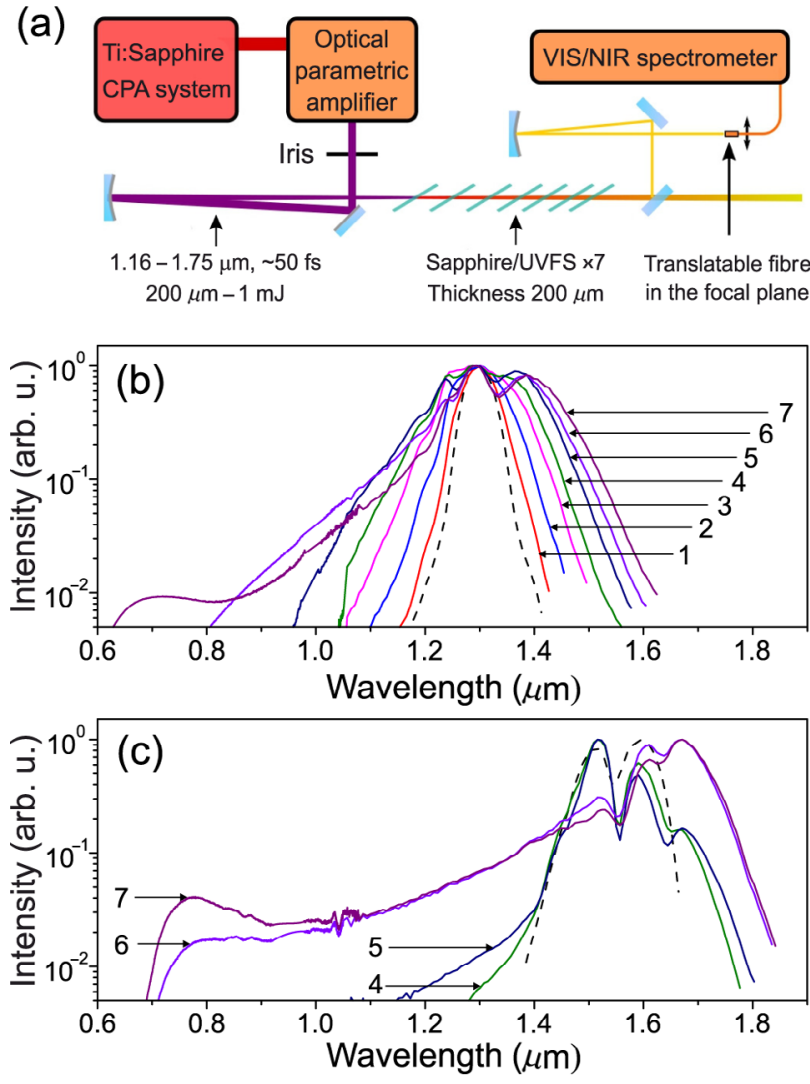

Fig. 21. (a) Experimental setup for supercontinuum generation in multiple alternating plates of UV-grade fused silica and sapphire. Supercontinuum spectra as functions of the plate number recorded with the input pulse wavelengths of (b) $1.3 \mu \mathrm{m}$ and (c) $1.6 \mu \mathrm{m}$, which fall into the ranges of zero and anomalous GVD, respectively. The input spectra are shown by dashed curves. The input pulses energies were $0.36 \mathrm{~mJ}$ at $1.3 \mu \mathrm{m}$ and $0.5 \mathrm{~mJ}$ at $1.6 \mu \mathrm{m}$. Adapted from [283]. 
essential performance characteristics, such as shot-to-shot energy stability and stability of the carrier envelope phase of the output pulses. Finally, the numerical simulations predict that multiple thin plates of a solid-state medium separated by appropriate spatial filters could be employed to broaden the spectrum of ultrahigh peak power pulses, yielding spectral bandwidths that are compressible to few-cycle pulsewidths, with undistorted output beam profiles that are focusable to ultrarelativistic intensities [284].

An even more simple and thus very attractive approach for extracavity pulse compression makes use of the spectral broadening in bulk solid-state media featuring the anomalous GVD. In contrast, here no additional dispersive element to compensate for the self-phase modulation is required: the job is done by the anomalous GVD of the medium itself. The numerical simulations suggest that mid-infrared pulses may be self-compressed down to a single optical cycle limit [80].

To this end, generation of filament-induced spatiotemporal light bullets, which are self-compressed down to or even below two optical cycles, was experimentally reported in various bulk solid-state dielectric media [84-87, 158, 195], as noted in the previous sections of the paper.

However, fully evolved self-compressed light bullets contain just a relatively small fraction of the input energy and exhibit a dispersive broadening as they leave the nonlinear medium and propagate in the free space [83], and these features may appear undesirable for a range of practical applications. Therefore a more attractive realization of the anomalous GVD-induced self-compression mechanism relies upon using a shorter nonlinear medium and a larger input beam, extracting the self-compressed pulse before the filamentation regime sets in. In that way, sub-3 optical cycle self-compressed pulses with $>10 \mu \mathrm{J}$ energy were obtained at $3 \mu \mathrm{m}$ by placing the $3 \mathrm{~mm}$ thick YAG plate at the output of the high repetition rate OPCPA system [285, 286]. The self-compression of parametrically amplified difference frequency pulses in few-mm thick YAG, $\mathrm{CaF}_{2}$ and $\mathrm{BaF}_{2}$ crystals down to sub-3 optical cycle widths was demonstrated in the 3-4 $\mu \mathrm{m}$ spectral range [200]. Figure 22 presents the experimental results illustrating a three-fold self-compression of $70 \mathrm{fs}$ pulses with the carrier wavelength of $3 \mu \mathrm{m}$ down
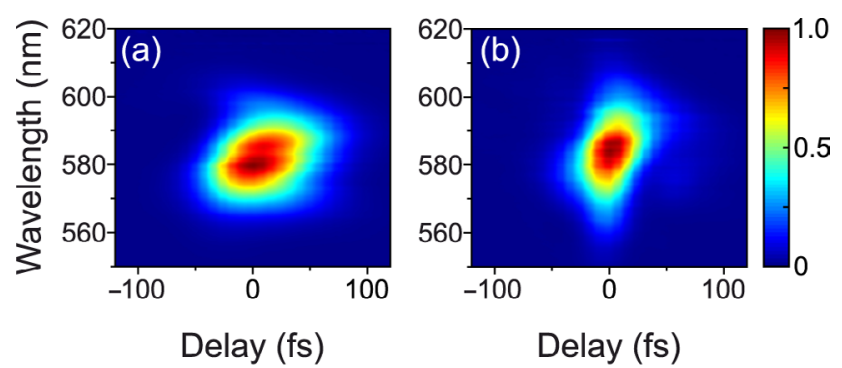

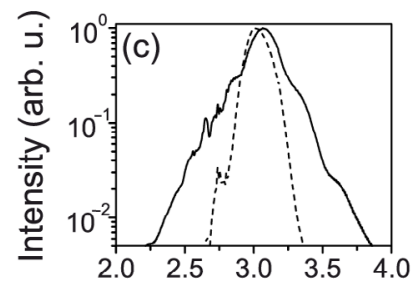

Wavelength $(\mu \mathrm{m})$

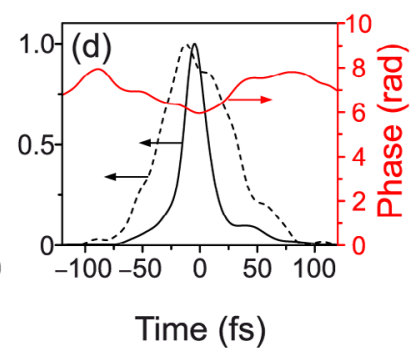

Fig. 22. Self-compression of $70 \mathrm{fs}, 3 \mu \mathrm{m}$ pulses in a $3 \mathrm{~mm}$-thick YAG plate. Measured SFG-FROG traces of (a) input, (b) self-compressed pulses, (c) spectrum and $(\mathrm{d})$ retrieved intensity profile and phase of the self-compressed pulse. Dashed curves in (c) and (d) show the spectrum and retrieved intensity profile of the input pulse. Adapted from [200].

to $23 \mathrm{fs}$ ( 2.3 optical cycles) in a $3 \mathrm{~mm}$-thick YAG plate, due to the interplay between the self-phase modulation and anomalous GVD and without the onset of self-focusing effects. An energy throughput efficiency of above $90 \%$ was measured, yielding the self-compressed pulses with sub-30 $\mu \mathrm{J}$ energy, with the only energy losses occurring due to Fresnel reflections from the input and output faces of the nonlinear crystal.

Prospects for wavelength and power scaling of the self-compression technique were investigated numerically [287-289, suggesting that scaling to milijoule energies and terawatt peak powers is readily feasible as long as one dimensional dynamics of the input pulse are maintained and small-scale filamentation of large beams is avoided [290, 291]. More recently, the numerical predictions were verified experimentally, where sub-three-cycle (30 fs) multimilijoule pulses with $0.44 \mathrm{TW}$ peak power were compressed in the YAG crystal of $2 \mathrm{~mm}$ thickness and extracted before the onset of modulation instability and multiple filamentation, as a result of a favourable interplay between a strong anomalous GVD and an optical nonlinearity around the carrier wavelength of $3.9 \mu \mathrm{m}$ [292. 


\section{Other developments}

A different mechanism of pulse compression and spectral broadening was uncovered for the propagation of intense (few $\mathrm{TW} / \mathrm{cm}^{2}$ ) mid-infrared pulses in a thin piece of the nonlinear medium $\left(\sim 2 \mathrm{~mm} \mathrm{CaF}_{2}\right.$ plate $)$ [293]. Here the self-compression of the driving pulse was shown to occur due to the generation of free electron plasma, which expels the trailing part of the pulse out of the propagation axis. The plasma-induced pulse compression resulted in the spectral broadening around the carrier wavelength as well as facilitated the large scale spectral broadening of the third, fifth and seventh harmonics via a cross phase modulation, eventually yielding more than 4-octave spanning SC from the ultraviolet to the midinfrared. Interestingly, the overlapping spectra of odd harmonics produce a remarkable spectral extension into the deep-ultraviolet, which is well beyond the blue-shifted cut-off constrained by the material GVD [61]. Plasma-induced compression along with broadband four-wave mixing was shown to dramatically enhance the SC generation in GaAs around its zero-GVD wavelength $(6.8 \mu \mathrm{m})$ [294. Post-compression of an octave spanning spectrum in a $0.5-\mathrm{mm} \mathrm{BaF}_{2}$ plate yielded a $20 \mathrm{fs}$ pulse at the output, that is less than 0.9 field cycles.

Only a moderate broadening of the SC spectrum was observed with the Ti:sapphire laser pumping in narrow bandgap dielectric crystals, such as diamond [295]. However, recent numerical simulations predict that the filamentation of femtosecond mid-infrared pulses in medium and narrow bandgap dielectric media, which possess an extremely broad mid-infrared transmittance and whose zero GVD points are located deeply in the mid-infrared, might produce the multi-octave SC spectra with a remarkable red-shifted spectral broadening [296]. For instance, the simulated SC spectra in sodium chloride and potassium iodide, using the pump wavelength of $5 \mu \mathrm{m}$, cover wavelength ranges of $0.7-7.6 \mu \mathrm{m}$ and $0.66-22 \mu \mathrm{m}$, respectively, suggesting these media as potentially attractive alternatives to soft glasses and semiconductor crystals. An interesting experimental study on SC generation with $1.24 \mu \mathrm{m}$ femtosecond pulses from the Cr:forsterite laser was performed in various aggregate states of $\mathrm{CO}_{2}$ : high-pressure gas, liquid, and supercritical fluid [297]. The authors reported SC generation in the $0.4-2.2 \mu \mathrm{m}$ range, whose spectral shape was strongly dependent on the pressure.

So far, the vast majority of experiments on SC generation have been performed using conventional, Gaussian-shaped input beams. However, several studies were devoted to investigate the SC generation with more complex input waveforms. In that regard, SC generation was studied using femtosecond Bessel beams with various parameters: cone angle, central spot size, energy content, particular intensity variation along the propagation axis and length of the Bessel zone. Self-focusing of ultrashort-pulsed Bessel beams was investigated in various nonlinear media, such as water [298], methanol [299], sapphire [30] and $\mathrm{BaF}_{2}$ [300, 301], and demonstrated that the SC radiation appears on the propagation axis. However, due to energy distribution between the central spot and surrounding rings, Bessel beams are very resistant to self-focusing, and 10-100 times higher incident energy is required to induce filamentation and achieve an appreciable spectral broadening. Filamentation and SC generation with self-bending Airy beams in water [302, 303] and in fused silica [304] have shown that the patterns of SC emission provide quantitative clues on the complex evolution of these sophisticated waveforms in the highly nonlinear propagation regime. Finally, the SC generation was investigated in $\mathrm{CaF}_{2}$ with vortex [305] and singular [306] beams, which are associated with the presence of spiral and step-wise phase dislocations in the wavefront of a beam, respectively, and which determine its phase and intensity structure. In both cases it was shown that the generated SC experiences large divergence and appears as a wide white-light background surrounding the original input beam, whose spatial profile remains well preserved in the process of SC generation.

\section{Conclusions}

To summarize, the present state-of-the-art ultrafast SC generation in bulk condensed media has reached a high level of maturity thanks to a wealth of new exciting experimental results, which were backed-up by in-depth understanding of femtosecond filamentation phenomena. 
The advances in SC generation in bulk solid-state media boosted the development of all-solid-state laser technology, which made high power few optical cycle pulses accessible throughout the optical range. Such pulses attract a great scientific and technological interest and will continue to do so in the future, extending the ultrafast nonlinear optics and laser spectroscopy to new, previously inaccessible domains. In particular, SC generation represents one of the fundamental building blocks of the emerging so-called third-generation femtosecond technology, which foresees boosting the peak and average powers of few optical cycle pulses simultaneously to the multiterawatt and hundreds of watts range, respectively, paving the way for the generation of powerful sub-cycle pulses with full control over the generated light waves in the optical range and beyond [307].

\section{Acknowledgements}

We are particularly indebted to prof. E. Riedle, prof.E. Mazur, prof. M. Vengris, dr. A. Varanavičius and R. Budriūnas for their kind permissions to reproduce some of their data. We also thank N. Garejev and A. Marcinkevičūte for their help in performing the measurements dedicated to illustrate important issues discussed throughout the paper. This work was funded by the Research Council of Lithuania, Grant APP-8/2016.

\section{References}

[1] J.M. Dudley, G. Genty, and S. Coen, Supercontinuum generation in photonic crystal fiber, Rev. Mod. Phys. 78, 1135-1184 (2006).

[2] J.H.V. Price, X. Feng, A.M. Heidt, G. Brambilla, P. Horak, F. Poletti, G. Ponzo, P. Petropoulos, M. Petrovich, J. Shi, M. Ibsen, W.H. Loh, H.N. Rutt, and D.J. Richardson, Supercontinuum generation in non-silica fibers, Opt. Fiber Technol. 18, 327-344 (2012).

[3] P. Domachuk, N.A. Wolchover, M. CroninGolomb, A. Wang, A.K. George, C.M.B. Cordeiro, J.C. Knight, and F.G. Omenetto, Over 4000 nm bandwidth of mid-IR supercontinuum generation in sub-centimeter segments of highly nonlinear tellurite PCFs, Opt. Express 16, 7161-7168 (2008).
[4] C.R. Petersen, U. Moller, I. Kubat, B. Zhou, S. Dupont, J. Ramsay, T. Benson, S. Sujecki, N. Abdel-Moneim, Z. Tang, D. Furniss, A. Seddon, and O. Bang, Mid-infrared supercontinuum covering the 1.4-13.3 $\mu \mathrm{m}$ molecular fingerprint region using ultra-high NA chalcogenide step-index fibre, Nature Photon. 8, 830-834 (2014).

[5] D. Kartashov, S. Ališauskas, A. Pugžlys, A. Voronin, A. Zheltikov, M. Petrarca, P. Béjot, J. Kasparian, J.-P. Wolf, and A. Baltuška, White light generation over three octaves by femtosecond filament at $3.9 \mu \mathrm{m}$ in argon, Opt. Lett. 37, 34563458 (2012).

[6] A.V. Mitrofanov, A.A. Voronin, S.I. Mitryukovskiy, D.A. Sidorov-Biryukov, A. Pugžlys, G. Andriukaitis, T. Flöry, E.A. Stepanov, A.B. Fedotov, A. Baltuška, and A.M. Zheltikov, Mid-infraredto-mid-ultraviolet supercontinuum enhanced by third-to-fifteenth odd harmonics, Opt. Lett. 40, 2068-2071 (2015).

[7] R.R. Alfano and L. Shapiro, Emission in the region 4000 to $7000 \AA$ via four photon coupling in glass, Phys. Rev. Lett. 24, 584-587 (1970).

[8] R.R. Alfano and L. Shapiro, Observation of selfphase modulation and small-scale filaments in crystals and glasses, Phys. Rev. Lett. 24, 592-594 (1970).

[9] R.R. Alfano, ed., The Supercontinuum Laser Source (Springer, 2006).

[10]R.L. Fork, C.V. Shank, C. Hirlimann, R. Yen, and W.J. Tomlinson, Femtosecond white-light continuum pulses, Opt. Lett. 8, 1-3 (1983).

[11]M. Witmann and A. Penzkofer, Spectral superbroadening of femtosecond laser pulses, Opt. Commun. 126, 308-317 (1996).

[12]D.E. Spence, P.N. Kean, and W. Sibbett, 60fsec pulse generation from a self-mode-locked Ti:sapphire laser, Opt. Lett. 16, 42-44 (1991).

[13]T.B. Norris, Femtosecond pulse amplification at $250 \mathrm{kHz}$ with a Ti:sapphire regenerative amplifier and application to continuum generation, Opt. Lett. 17, 1009-1011 (1992).

[14]S. Backus, C.G. Durfee, M.M. Murnane, and H.C. Kapteyn, High power ultrafast lasers, Rev. Sci. Instr. 69, 1207-1223 (1998). 
[15]A. Brodeur and S.L. Chin, Band-gap dependence of the ultrafast white-light continuum, Phys. Rev. Lett. 80, 4406-4409 (1998).

[16]A. Brodeur and S.L. Chin, Ultrafast white-light continuum generation and self-focusing in transparent condensed media, J. Opt. Soc. Am. B 16, 637-650 (1999).

[17]K.R. Wilson and V.V. Yakovlev, Ultrafast rainbow: Tunable ultrashort pulses from a solid-state kilohertz system, J. Opt. Soc. Am. B 14, 444-448 (1997).

[18]A. Couairon, V. Jukna, J. Darginavičius, D. Majus, N. Garejev, I. Gražulevičiūtè, G. Valiulis, G. Tamošauskas, A. Dubietis, F. Silva, et al., in: Laser Filamentation, CRM Series in Mathematical Physics, eds. A.D. Bandrauk et al. (Springer, 2016) pp. 147-165.

[19]S.L. Chin, S. Petit, F. Borne and K. Miyazaki, The white light supercontinuum is indeed an ultrafast white light laser, Jpn. J. Appl. Phys. 38, L126-128 (1999).

[20]W. Watanabe and K. Itoh, Spatial coherence of supercontinuum emitted from multiple filaments, Jpn. J. Appl. Phys. 40, 592-595 (2001).

[21]B. Prade, M. Franco, A. Mysyrowicz, A. Couairon, H. Buersing, B. Eberle, M. Krenz, D. Seiffer, and O. Vasseur, Spatial mode cleaning by femtosecond filamentation in air, Opt. Lett. 31, 2601-2603 (2006).

[22]D. Wegkamp, D. Brida, S. Bonora, G. Cerullo, J. Stähler, M. Wolf, and S. Wall, Phase retrieval and compression of low-power white-light pulses, Appl. Phys. Lett. 99, 101101 (2011).

[23]K. Midorikawa, H. Kawano, A. Suda, C. Nagura, and M. Obara, Polarization properties of ultrafast white-light continuum generated in condensed media, Appl. Phys. Lett. 80, 923-925 (2002).

[24]A.K. Dharmadhikari, F.A. Rajgara, and D. Mathur, Depolarization of white light generated by ultrashort laser pulses in optical media, Opt. Lett. 31, 2184-2186 (2006).

[25]R.S.S. Kumar, K.L.N. Deepak, and D.N. Rao, Depolarization properties of the femtosecond supercontinuum generated in condensed media, Phys. Rev. A 78, 043818 (2008).
[26]M. Bradler, P. Baum, and E. Riedle, Femtosecond continuum generation in bulk laser host materials with sub- $\mu$ J pump pulses, Appl. Phys. B 97, 561-574 (2009).

[27]U. Megerle, I. Pugliesi, C. Schriever, C.F. Sailer, and E. Riedle, Sub-50 fs broadband absorption spectroscopy with tunable excitation: putting the analysis of ultrafast molecular dynamics on solid ground, Appl. Phys. B 96, 215-231 (2009).

[28]D. Majus, V. Jukna, E. Pileckis, G. Valiulis, and A. Dubietis, Rogue-wave-like statistics in ultrafast white-light continuum generation in sapphire, Opt. Express 19, 16317-16323 (2011).

[29]A. van de Walle, M. Hanna, F. Guichard, Y. Zaouter, A. Thai, N. Forget, and P. Georges, Spectral and spatial fullbandwidth correlation analysis of bulk-generated supercontinuum in the mid-infrared, Opt. Lett. 40, 673-675 (2015).

[30]D. Majus and A. Dubietis, Statistical properties of ultrafast supercontinuum generated by femtosecond Gaussian and Bessel beams: a comparative study, J. Opt. Soc. Am. B 30, 994-999 (2013).

[31]M. Bradler and E. Riedle, Temporal and spectral correlations in bulk continua and improved use in transient spectroscopy, J. Opt. Soc. Am. B 31, 1465-1475 (2014).

[32]G. Auböck, C. Consani, R. Monni, A. Cannizzo, F. van Mourik, and M. Chergui, Femtosecond pump/supercontinuumprobe setup with $20 \mathrm{kHz}$ repetition rate, Rev. Sci. Instrum. 83, 093105 (2012).

[33]C. Calabrese, A.M. Stingel, L. Shen, and P.B. Petersen, Ultrafast continuum mid-infrared spectroscopy: probing the entire vibrational spectrum in a single laser shot with femtosecond time resolution, Opt. Lett. 37, 2265-2267 (2012).

[34]E. Riedle, M. Bradler, M. Wenninger, C.F. Sailer, and I. Pugliesi, Electronic transient spectroscopy from the deep-UV to the NIR: unambiguous disentanglement of complex processes, Faraday Discuss. 163, 139-158 (2013).

[35]M. Balu, J. Hales, D.J. Hagan, and E.W. Van Stryland, White-light continuum Z-scan technique for nonlinear materials characterization, Opt. Express 12, 3820-3826 (2004). 
[36]L. De Boni, A.A. Andrade, L. Misoguti, C. Mendonça, and S.C. Zilio, Z-scan measurements using femtosecond continuum generation, Opt. Express 12, 3921-3927 (2004).

[37]K. Cook, A.K. Kar, and R.A. Lamb, White-light supercontinuum interference of self-focused filaments in water, Appl. Phys. Lett. 83, 3861-3863 (2003).

[38]C. Corsi, A. Tortora, and M. Bellini, Generation of a variable linear array of phase-coherent supercontinuum sources, Appl. Phys. B 78, 299-304 (2004).

[39]M. Bellini and T.W. Hänsch, Phase-locked whitelight continuum pulses: toward a universal optical frequency-comb synthesizer, Opt. Lett. 25, 1049-1051 (2000).

[40]M. Bellini and T.W. Hänsch, Generation and applications of phase-locked white-light continuum pulses, Laser Part. Beams 19, 157-159 (2001).

[41]A. Tortora, C. Corsi, and M. Bellini, Comb-like supercontinuum generation in bulk media, Appl. Phys. Lett. 85, 1113-1115 (2004).

[42]P. Baum, S. Lochbrunner, J. Piel, and E. Riedle, Phase-coherent generation of tunable visible femtosecond pulses, Opt. Lett. 28, 185-187 (2003).

[43]P. Baum, E. Riedle, M. Greve, and H.R. Telle, Phase-locked ultrashort pulse trains at separate and independently tunable wavelengths, Opt. Lett. 30, 2028-2030 (2005).

[44]Y. Liu, Y. Brelet, Z. He, L. Yu, S. Mitryukovskiy, A. Houard, B. Forestier, A. Couairon, and A. Mysyrowicz, Ciliary white light: optical aspect of ultrashort laser ablation on transparent dielectrics, Phys. Rev. Lett. 110, 097601 (2013).

[45]G. Cerullo and S. De Silvestri, Ultrafast optical parametric amplifiers, Rev. Sci. Instrum. 74, 1-18 (2003).

[46]T. Wilhelm, J. Piel, and E. Riedle, Sub-20-fs pulses tunable across the visible from a blue-pumped single pass noncollinear parametric converter, Opt. Lett. 22, 1494-1496 (1997).

[47]D. Brida, C. Manzoni, G. Cirmi, M. Marangoni, S. Bonora, P. Villoresi, S. De Silvestri, and G. Cerullo, Few-optical-cycle pulses tunable from the visible to the mid-infrared by optical parametric amplifiers, J. Opt. 12, 013001 (2010).
[48]C. Manzoni, G. Cirmi, D. Brida, S. De Silvestri, and G. Cerullo, Optical-parametric-generation process driven by femtosecond pulses: Timing and carrier-envelope phase properties, Phys. Rev. A 79, 033818 (2009).

[49]A. Dubietis, R. Butkus, and A.P. Piskarskas, Trends in chirped pulse optical parametric amplification, IEEE J. Sel. Top. Quant. Electron. 12, 163-172 (2006).

[50]A. Harth, M. Schultze, T. Lang, T. Binhammer, S. Rausch, and U. Morgner, Two-color pumped OPCPA system emitting spectra spanning 1.5 octaves from VIS to NIR, Opt. Express 20, 30763081 (2012).

[51]M. Schulz, R. Riedel, A. Willner, T. Mans, C. Schnitzler, P. Russbueldt, J. Dolkemeyer, E. Seise, T. Gottschall, S. Hädrich, et al., Yb:YAG Innoslab amplifier: efficient high repetition rate subpicosecond pumping system for optical parametric chirped pulse amplification, Opt. Lett. 36, 2456-2458 (2011).

[52]R. Budriūnas, T. Stanislauskas, J. Adamonis, A. Aleknavičius, G. Veitas, D. Gadonas, S. Balickas, A. Michailovas, and A. Varanavičius, $53 \mathrm{~W}$ average power CEP-stabilized OPCPA system delivering $5.5 \mathrm{TW}$ few cycle pulses at $1 \mathrm{kHz}$ repetition rate, Opt. Express 25, 5797-5806 (2017).

[53]S.L. Chin, S.A. Hosseini, W. Liu, Q. Luo, F. Thberge, N. Aközbek, A. Becker, V.P. Kandidov, O.G. Kosareva, and H. Schroeder, The propagation of powerful femtosecond laser pulses in optical media: physics, applications, and new challenges, Can. J. Phys. 83, 863-905 (2005).

[54]A. Couairon and A. Mysyrowicz, Femtosecond filamentation in transparent media, Phys. Rep. 441, 47-190 (2007).

[55]L. Bergé, S. Skupin, R. Nuter, J. Kasparian, and J.-P. Wolf, Ultrashort filaments of light in weakly ionized, optically transparent media, Rep. Prog. Phys. 70, 1633-1713 (2007).

[56] V.P. Kandidov, S.A. Shlenov, and O.G. Kosareva, Filamentation of high-power femtosecond laser radiation, Quantum Electron. 39, 205-228 (2009).

[57]J.H. Marburger, Self-focusing: Theory, Prog. Quantum Electron. 4, 35-110 (1975). 
[58]A. Dubietis, A. Couairon, E. Kučinskas, G. Tamošauskas, E. Gaižauskas, D. Faccio, and P. Di Trapani, Measurement and calculation of nonlinear absorption associated with femtosecond filaments in water, Appl. Phys. B 84, 439-446 (2006).

[59]M. Sheik-Bahae, D.J. Hagan, and E.W. Van Stryland, Dispersion and band-gap scaling of the electronic Kerr effect in solids associated with two-photon absorption, Phys. Rev. Lett. 65, 9699 (1990).

[60]W. Liu, S. Petit, A. Becker, N. Aközbek, C.M. Bowden, and S.L. Chin, Intensity clamping of a femtosecond laser pulse in condensed matter, Opt. Commun. 202, 189-197 (2002).

[61]M. Kolesik, G. Katona, J.V. Moloney, and E.M. Wright, Physical factors limiting the spectral extent and band gap dependence of supercontinuum generation, Phys. Rev. Lett. 91, 043905 (2003).

[62]M. Kolesik, G. Katona, J.V. Moloney, and E.M. Wright, Theory and simulation of supercontinuum generation in transparent bulk media, Appl. Phys. B 77, 185-195 (2003).

[63]S. Skupin and L. Bergé, Self-guiding of femtosecond light pulses in condensed media: Plasma generation versus chromatic dispersion, Physica D 220, 14-30 (2006).

[64]P. Chernev and V. Petrov, Self-focusing of light pulses in the presence of normal group-velocity dispersion, Opt. Lett. 17, 172-174 (1992).

[65]J.E. Rothenberg, Pulse splitting during self-focusing in normally dispersive media, Opt. Lett. 17, 583-585 (1992).

[66]J.E. Rothenberg, Space-time focusing: breakdown of the slowly varying envelope approximation in the self-focusing of femtosecond pulses, Opt. Lett. 17, 1340-1342 (1992).

[67]G. Fibich and G.C. Papanicolaou, Self-focusing in the presence of small time dispersion and nonparaxiality, Opt. Lett. 22, 1397-1399 (1997).

[68]J.K. Ranka, R.W. Schirmer, and A.L. Gaeta, Observation of pulse splitting in nonlinear dispersive media, Phys. Rev. Lett. 77, 3783-3786 (1996).
[69]J.K. Ranka and A.L. Gaeta, Breakdown of the slowly varying envelope approximation in the self-focusing of ultrashort pulses, Opt. Lett. 23, 534-536 (1998).

[70]S.A. Diddams, H.K. Eaton, A.A. Zozulya, and T.S. Clement, Amplitude and phase measurements of femtosecond pulse splitting in nonlinear dispersive media, Opt. Lett. 23, 379-381 (1998).

[71]A.A. Zozulya, S.A. Diddams, A.G. Van Engen, and T.S. Clement, Propagation dynamics of intense femtosecond pulses: multiple splittings, coalescence, and continuum generation, Phys. Rev. Lett. 82, 1430-1433 (1999).

[72]A.L. Gaeta, Catastrophic collapse of ultrashort pulses, Phys. Rev. Lett. 84, 3582-3585 (2000).

[73]A.L. Gaeta, Spatial and temporal dynamics of collapsing ultrashort laser pulses, Topics Appl. Phys. 114, 399-412 (2009).

[74]Y. Silberberg, Collapse of optical pulses, Opt. Lett. 15, 1282-1284 (1990).

[75]L. Bergé and S. Skupin, Self-channeling of ultrashort laser pulses in materials with anomalous dispersion, Phys. Rev. E 71, 065601(R) (2005).

[76]J. Liu, R. Li, and Z. Xu, Few-cycle spatiotemporal soliton wave excited by filamentation of a femtosecond laser pulse in materials with anomalous dispersion, Phys. Rev. A 74, 043801 (2006).

[77]S.V. Chekalin, V.O. Kompanets, E.O. Smetanina, and V.P. Kandidov, Light bullets and supercontinuum spectrum during femtosecond pulse filamentation under conditions of anomalous group-velocity dispersion in fused silica, Quant. Electron. 43, 326-331 (2013).

[78]K.D. Moll and A.L. Gaeta, Role of dispersion in multiple-collapse dynamics, Opt. Lett. 29, 995997 (2004).

[79]A. Saliminia, S.L. Chin, and R. Vallée, Ultrabroad and coherent white light generation in silica glass by focused femtosecond pulses at $1.5 \mu \mathrm{m}$, Opt. Express 13, 5731-5738 (2005).

[80]F. Silva, D.R. Austin, A. Thai, M. Baudisch, M. Hemmer, D. Faccio, A. Couairon, and J. Biegert, Multi-octave supercontinuum generation from mid-infrared filamentation in a bulk crystal, Nat. Commun. 3, 807 (2012). 
[81]M. Durand, A. Jarnac, A. Houard, Y. Liu, S. Grabielle, N. Forget, A. Durécu, A. Couairon, and A. Mysyrowicz, Selfguided propagation of ultrashort laser pulses in the anomalous dispersion region of transparent solids: a new regime of filamentation, Phys. Rev. Lett. 110, 115003 (2013).

[82]E.O. Smetanina, V.O. Kompanets, A.E. Dormidonov, S.V. Chekalin, and V.P. Kandidov, Light bullets from near-IR filament in fused silica, Laser Phys. Lett. 10, 105401 (2013).

[83]D. Majus, G. Tamošauskas, I. Gražulevičiūtè, N. Garejev, A. Lotti, A. Couairon, D. Faccio, and A. Dubietis, Nature of spatiotemporal light bullets in bulk Kerr media, Phys. Rev. Lett. 112, 193901 (2014).

[84]I. Gražulevičiūtė, R. Šuminas, G. Tamošauskas, A. Couairon, and A. Dubietis, Carrier-envelope phase-stable spatiotemporal light bullets, Opt. Lett. 40, 3719-3722 (2015).

[85]S.V. Chekalin, A.E. Dokukina, A.E. Dormidonov, V.O. Kompanets, E.O. Smetanina, and V.P. Kandidov, Light bullets from a femtosecond filament, J. Phys. B 48, 094008 (2015).

[86]R. Šuminas, G. Tamošauskas, G. Valiulis, and A. Dubietis, Spatiotemporal light bullets and supercontinuum generation in $\beta$-BBO crystal with competing quadratic and cubic nonlinearities, Opt. Lett. 41, 2097-2100 (2016).

[87]I. Gražulevičiūtė, N. Garejev, D. Majus, V. Jukna, G. Tamošauskas, and A. Dubietis, Filamentation and light bullet formation dynamics in solid-state dielectric media with weak, moderate and strong anomalous group velocity dispersion, J. Opt. 18, 025502 (2016).

[88]M. Kolesik, E.M. Wright, and J.V. Moloney, Interpretation of the spectrally resolved far field of femtosecond pulses propagating in bulk nonlinear dispersive media, Opt. Express 13, 1072910741 (2005).

[89]D. Faccio, P. Di Trapani, S. Minardi, A. Bramati, F. Bragheri, C. Liberale, V. Degiorgio, A. Dubietis, and A. Matijosius, Far-field spectral characterization of conical emission and filamentation in Kerr media, J. Opt. Soc. Am. B 22, 862-869 (2005).

[90]M.A. Porras, A. Dubietis, E. Kučinskas, F. Bragheri, V. Degiorgio, A. Couairon, D. Faccio, and
P. Di Trapani, From X- to O-shaped spatiotemporal spectra of light filaments in water, Opt. Lett. 30, 3398-3400 (2005).

[91]D. Faccio, A. Averchi, A. Lotti, M. Kolesik, J.V. Moloney, A. Couairon, and P. Di Trapani, Generation and control of extreme blueshifted continuum peaks in optical Kerr media, Phys. Rev. A 78, 033825 (2008).

[92]D. Faccio, M. Clerici, A. Averchi, A. Lotti, O. Jedrkiewicz, A. Dubietis, G. Tamosauskas, A. Couairon, F. Bragheri, D. Papazoglou, S. Tzortzakis, and P. Di Trapani, Few-cycle laser-pulse collapse in Kerr media: the role of group-velocity dispersion and X-wave formation, Phys. Rev. A 78, 033826 (2008).

[93]M. Kolesik, E.M. Wright, and J.V. Moloney, Dynamic nonlinear $\mathrm{X}$ waves for femtosecond pulse propagation in water, Phys. Rev. Lett. 92, 253901 (2004).

[94]A. Couairon, E. Gaižauskas, D. Faccio, A. Dubietis, and P. Di Trapani, Nonlinear X-wave formation by femtosecond filamentation in Kerr media, Phys. Rev. E 73, 016608 (2006).

[95]M.A. Porras, A. Parola, and P. Di Trapani, Nonlinear unbalanced $O$ waves: nonsolitary, conical light bullets in nonlinear dissipative media, J. Opt. Soc. Am. B 22, 1406-1413 (2005).

[96]A. Dubietis, E. Gaižauskas, G. Tamošauskas, and P. Di Trapani, Light filaments without self-channeling, Phys. Rev. Lett. 92, 253903 (2004).

[97]A. Dubietis, E. Kučinskas, G. Tamošauskas, E. Gaižauskas, M.A. Porras, and P. Di Trapani, Self-reconstruction of light filaments, Opt. Lett. 29, 2893-2895 (2004).

[98]I. Gražulevičiūtè, G. Tamošauskas, V. Jukna, A. Couairon, D. Faccio, and A. Dubietis, Selfreconstructing spatiotemporal light bullets, Opt. Express 22, 30613-30622 (2014).

[99]D. Faccio, M.A. Porras, A. Dubietis, F. Bragheri, A. Couairon, and P. Di Trapani, Conical emission, pulse splitting, and X-wave parametric amplification in nonlinear dynamics of ultrashort light pulses, Phys. Rev. Lett. 96, 193901 (2006).

[100] M. Kolesik and J.V. Moloney, Nonlinear optical pulse propagation simulation: From 
Maxwell's to unidirectional equations, Phys. Rev. E 70, 036604 (2004).

[101] A. Couairon, E. Brambilla, T. Corti, D. Majus, O. de J. Ramírez-Góngora, and M. Kolesik, Practitioner's guide to laser pulse propagation models and simulation, Eur. Phys. J. Spec. Top. 199, 5-76 (2011).

[102] A. Couairon, O.G. Kosareva, N.A. Panov, D.E. Shipilo, V.A. Andreeva, V. Jukna, and F. Nesa, Propagation equation for tight-focusing by a parabolic mirror, Opt. Express 23, 31240-31252 (2015).

[103] A.V. Husakou and J. Herrmann, Supercontinuum generation of higher-order solitons by fission in photonic crystal fibers, Phys. Rev. Lett. 87, 203901 (2001).

[104] T. Brabec and F. Krausz, Nonlinear optical pulse propagation in the single-cycle regime, Phys. Rev. Lett. 78, 3282-3285 (1997).

[105] M. Kolesik and J.V. Moloney, Modeling and simulation techniques in extreme nonlinear optics of gaseous and condensed media, Rep. Prog. Phys. 77, 016401 (2014).

[106] M.J. Weber, Handbook of Optical Materials (CRC Press, London, 2003).

[107] R. DeSalvo, A.A. Said, D.J. Hagan, E.W. Van Stryland, and M. Sheik-Bahae, Infrared to ultraviolet measurements of two-photon absorption and $n_{2}$ in wide-bandgap solids, IEEE J. Quantum Electron. 32, 1324-1333 (1996).

[108] H.H. Li, Refractive index of alkali halides and its wavelength and temperature derivatives, J. Phys. Chem. Ref. Data 5, 329-528 (1976).

[109] R. Adair, L.L. Chase, and S.A. Payne, Nonlinear refractive index of optical crystals, Phys. Rev. B 39, 3337-3350 (1989).

[110] H.H. Li, Refractive index of alkali halides and its wavelength and temperature derivatives, J. Phys. Chem. Ref. Data 9, 161-289 (1980).

[111] A. Major, F. Yoshino, I. Nikolakakos, J.S. Aitchison, and P.W.E. Smith, Dispersion of the nonlinear refractive index in sapphire, Opt. Lett. 29, 602-604 (2004).

[112] A. Couairon, L. Sudrie, M. Franco, B. Prade, and A. Mysyrowicz, Filamentation and damage in fused silica induced by tightly focused fem- tosecond laser pulses, Phys. Rev. B 71, 125435 (2005).

[113] D. Milam, Review and assessment of measured values of the nonlinear refractive-index coefficient of fused silica, Appl. Opt. 37, 546-550 (1998).

[114] I.H. Malitson, Interspecimen comparison of the refractive index of fused silica, J. Opt. Soc. Am. 55, 1205-1209 (1965).

[115] D.N. Nikogosyan, Nonlinear Optical Crystals: A Complete Survey (Springer Science \& Business Media, New York, 2005).

[116] F. Zernike, Refractive indices of ammonium dihydrogen phosphate and potassium dihydrogen phosphate between $2000 \AA$ and $1.5 \mu \mathrm{m}$, J. Opt. Soc. Am. 54, 1215-1220 (1964).

[117] J.V. Coe, A.D. Earhart, M.H. Cohen, G.J. Hoffman, H.W. Sarkas, and K.H. Bowen, Using cluster studies to approach the electronic structure of bulk water: Reassessing the vacuum level, conduction band edge, and band gap of water, J. Chem. Phys. 107, 6023-6031 (1997).

[118] E.T.J. Nibbering, M.A. Franco. B.S. Prade, G. Grillon, C. Le Blanc, and A. Mysyrowicz, Measurements of the nonlinear refractive index of transparent materials by spectral analysis after nonlinear propagation, Opt. Commun. 119, 479-484 (1995).

[119] A.G. Van Engen, S.A. Diddams, and T.S. Clement, Dispersion measurements of water with white-light interferometry, Appl. Opt. 37, 5679-5686 (1998).

[120] Y. Xu and W.Y. Ching, Electronic structure of yttrium aluminum garnet $\left(\mathrm{Y}_{3} \mathrm{Al}_{5} \mathrm{O}_{12}\right)$, Phys. Rev. B 59, 10530-10535 (1999).

[121] D.E. Zelmon, D.L. Small, and R. Page, Refractive-index measurements of undoped yttrium aluminum garnet from 0.4 to $5.0 \mu \mathrm{m}$, Appl. Opt. 37, 4933-4935 (1998).

[122] M. Bache, H. Guo, B. Zhou, and X. Zeng, The anisotropic Kerr nonlinear refractive index of the beta-barium borate $\left(\beta-\mathrm{BaB}_{2} \mathrm{O}_{4}\right)$ nonlinear crystal, Opt. Mater. Express 3, 357-382 (2013).

[123] D. Zhang, Y. Kong, and J.Y. Zhang, Optical parametric properties of 532-nm-pumped betabarium-borate near the infrared absorption edge, Opt. Commun. 184, 485-491 (2000). 
[124] D.J. Little, M. Ams, and M.J. Withford, Influence of bandgap and polarization on photoionization: guidelines for ultrafast laser inscription, Opt. Mater. Express 1, 670-677 (2011).

[125] SCHOTT Optical Glass Data Sheets (2015).

[126] A. Major, I. Nikolakakos, J.S. Aitchison, A.I. Ferguson, N. Langford, and P.W.E. Smith, Characterization of the nonlinear refractive index of the laser crystal $\mathrm{Yb}: \mathrm{KGd}\left(\mathrm{WO}_{4}\right)_{2}$, Appl. Phys. B 77, 433-436 (2003).

[127] C.E. Webb and J.D. Jones, Handbook of Laser Technology and Applications: Laser Design and Laser Systems, Vol. 2 (CRC Press, 2004).

[128] A.G. Selivanov, I.A. Denisov, N.V. Kuleshov, and K.V. Yumashev, Nonlinear refractive properties of $\mathrm{Yb}^{3+}$-doped $\mathrm{KY}\left(\mathrm{WO}_{4}\right)_{2}$ and $\mathrm{YVO}_{4}$ laser crystals, Appl. Phys. B 83, 61-65 (2006).

[129] M.C. Pujol, M. Rico, C. Zaldo, R. Solé, V. Nikolov, X. Solans, M. Aguiló, and F. Díaz, Crystalline structure and optical spectroscopy of $\mathrm{Er}^{3+}$-doped $\mathrm{KGd}\left(\mathrm{WO}_{4}\right)_{2}$ single crystals, Appl. Phys. B 68, 187-197 (1999).

[130] M.R. Dolgos, A.M. Paraskos, M.W. Stoltzfus, S.C. Yarnell, and P.M. Woodward, The electronic structures of vanadate salts: cation substitution as a tool for band gap manipulation, J. Solid State Chem. 182, 1964-1971 (2009).

[131] N.T. Nguyen, A. Saliminia, W. Liu, S.L. Chin, and R. Valée, Optical breakdown versus filamentation in fused silica by use of femtosecond infrared laser pulses, Opt. Lett. 28, 1591-1593 (2003).

[132] J.B. Ashcom, R.R. Gattass, C.B. Schaffer, and E. Mazur, Numerical aperture dependence of damage and supercontinuum generation from femtosecond laser pulses in bulk fused silica, J. Opt. Soc. Am. B 23, 2317-2322 (2006).

[133] V. Jukna, J. Galinis, G. Tamošauskas, D. Majus, and A. Dubietis, Infrared extension of femtosecond supercontinuum generated by filamentation in solid-state media, Appl. Phys. B 116, 477-483 (2014).

[134] Z.X. Wu, H.B. Jiang, L. Luo, H.C. Guo, H. Yang, and Q.H. Gong, Multiple foci and a long filament observed with focused femtosecond pulse propagation in fused silica, Opt. Lett. 27, 448-450 (2002).
[135] W. Liu, S.L. Chin, O. Kosareva, I.S. Golubtsov, and V.P. Kandidov, Multiple refocusing of a femtosecond laser pulse in a dispersive liquid (methanol), Opt. Commun. 225, 193-209 (2003).

[136] A.K. Dharmadhikari, J.A. Dharmadhikari, and D. Mathur, Visualization of focusing-refocusing cycles during filamentation in $\mathrm{BaF}_{2}, \mathrm{Appl}$. Phys. B 94, 259-263 (2009).

[137] M. Mlejnek, E.M. Wright, and J.V. Moloney, Dynamic spatial replenishment of femtosecond pulses propagating in air, Opt. Lett. 23, 382-384 (1998).

[138] A. Jarnac, G. Tamošauskas, D. Majus, A. Houard, A. Mysyrowicz, A. Couairon, and A. Dubietis, Whole life cycle of femtosecond ultraviolet filaments in water, Phys. Rev. A 89, 033809 (2014).

[139] A.V. Kuznetsov, V.O. Kompanets, A.E. Dormidonov, S.V. Chekalin, S.A. Shlenov, and V.P. Kandidov, Periodic colour-centre structure formed under filamentation of mid-IR femtosecond laser radiation in a LiF crystal, Quant. Electron. 46, 379-386 (2016).

[140] A. Dubietis, G. Tamošauskas, G. Fibich, and B. Ilan, Multiple filamentation induced by inputbeam ellipticity, Opt. Lett. 29, 1126-1128 (2004).

[141] D. Majus, V. Jukna, G. Tamošauskas, G. Valiulis, and A. Dubietis, Three-dimensional mapping of multiple filament arrays, Phys. Rev. A 81, 043811 (2010).

[142] L. Bergé, S. Mauger, and S. Skupin, Multifilamentation of powerful optical pulses in silica, Phys. Rev. A 81, 013817 (2010).

[143] C.B. Schaffer, A. Brodeur, and E. Mazur, Laser-induced breakdown and damage in bulk transparent materials induced by tightly focused femtosecond laser pulses, Meas. Sci. Technol. 12, 1784-1794 (2001).

[144] S. Tzortzakis, L. Sudrie, M. Franco, B. Prade, and A. Mysyrowicz, Self-guided propagation of ultrashort IR laser pulses in fused silica, Phys. Rev. Lett. 87, 213902 (2001).

[145] S. Tzortzakis, D.G. Papazoglou, and I. Zergioti, Long-range filamentary propagation of subpicosecond ultraviolet laser pulses in fused silica, Opt. Lett. 31, 796-798 (2006). 
[146] C. Nagura, A. Suda, H. Kawano, M. Obara, and K. Midorikawa, Generation and characterization of ultrafast white-light continuum in condensed media, Appl. Opt. 41, 3735-3742 (2002).

[147] X.-J. Fang and T. Kobayashi, Evolution of a super-broadened spectrum in a filament generated by an ultrashort intense laser pulse in fused silica, Appl. Phys. B 77, 167-170 (2003).

[148] H. Dachraoui, C. Oberer, M. Michelswirth, and U. Heinzmann, Direct time-domain observation of laser pulse filaments in transparent media, Phys. Rev. A 82, 043820 (2010).

[149] L. Zhang, T. Xi, Z. Hao, and J. Lin, Supercontinuum accumulation along a single femtosecond filament in fused silica, J. Phys. D 49, 115201 (2016).

[150] A.K. Dharmadhikari, F.A. Rajgara, and D. Mathur, Systematic study of highly efficient white-light generation in transparent materials using intense femtosecond pulses, Appl. Phys. B 80, 61-66 (2005).

[151] A.K. Dharmadhikari, F.A. Rajgara, and D. Mathur, Depolarization of white light generated by ultrashort laser pulses in optical media, Opt. Lett. 31, 2184-2186 (2006).

[152] J. Yang and G. Mu, Multi-dimensional observation of white-light filaments generated by femtosecond laser pulses in condensed medium, Opt. Express 15, 4943-4952 (2007).

[153] J. Jiang, Y. Zhong, Y. Zheng, Z. Zeng, X. Ge, and $\mathrm{R}$. Li, Broadening of white-light continuum by filamentation in BK7 glass at its zero-dispersion point, Phys. Lett. A 379, 1929-1933 (2015).

[154] M.L. Naudeau, R.J. Law, T.S. Luk, T.R. Nelson, and S.M. Cameron, Observation of nonlinear optical phenomena in air and fused silica using a $100 \mathrm{GW}, 1.54 \mu \mathrm{m}$ source, Opt. Express 14, 6194-6200 (2006).

[155] D. Faccio, A. Averchi, A. Couairon, A. Dubietis, R. Piskarskas, A. Matijošius, F. Bragheri, M.A. Porras, A. Piskarskas, and P. Di Trapani, Competition between phase-matching and stationarity in Kerr-driven optical pulse filamentation, Phys. Rev. E 74, 047603 (2006).

[156] M.A. Porras, A. Dubietis, A. Matijošius, R. Piskarskas, F. Bragheri, A. Averchi, and P. Di Trapani,
Characterization of conical emission of light filaments in media with anomalous dispersion, J. Opt. Soc. Am. B 24, 581-584 (2007).

[157] M.Durand, K.Lim, V. Jukna, E. McKee, M. Baudelet, A. Houard, M. Richardson, A. Mysyrowicz, and A. Couairon, Blueshifted continuum peaks from filamentation in the anomalous dispersion regime, Phys. Rev. A 87, 043820 (2013).

[158] E.O. Smetanina, V.O. Kompanets, S.V. Chekalin, A.E. Dormidonov, and V.P. Kandidov, AntiStokes wing of femtosecond laser filament supercontinuum in fused silica, Opt. Lett. 38, 16-18 (2013).

[159] S.V. Chekalin, V.O. Kompanets, A.E. Dokukina, A.E. Dormidonov, E.O. Smetanina, and V.P. Kandidov, Visible supercontinuum radiation of light bullets in the femtosecond filamentation of IR pulses in fused silica, Quant. Electron. 45, 401-407 (2015).

[160] I. Gražulevičiūtè, R. Šuminas, G. Tamošauskas, A. Couairon, and A. Dubietis, Carrierenvelope phase-stable spatiotemporal light bullets, Opt. Lett. 40, 3719-3722 (2015).

[161] J. Darginavičius, D. Majus, V. Jukna, N. Garejev, G. Valiulis, A. Couairon, and A. Dubietis, Ultrabroadband supercontinuum and third-harmonic generation in bulk solids with two optical-cycle carrier-envelope phase-stable pulses at $2 \mu \mathrm{m}$, Opt. Express 21, 25210-25220 (2013).

[162] J.A. Dharmadhikari, R.A. Deshpande, A. Nath, K. Dota, D. Mathur, and A.K. Dharmadhikari, Effect of group velocity dispersion on supercontinuum generation and filamentation in transparent solids, Appl. Phys. B 117, 471-479 (2014).

[163] N. Garejev, G. Tamošauskas, and A. Dubietis, Comparative study of multioctave supercontinuum generation in fused silica, YAG, and $\mathrm{LiF}$ in the range of anomalous group velocity dispersion, J. Opt. Soc. Am. B 34, 88-94 (2017).

[164] Y. Yang, M. Liao, X. Li, W. Bi, Y. Ohishi, T. Cheng, Y. Fang, G. Zhao, and W. Gao, Filamentation and supercontinuum generation in lanthanum glass, J. Appl. Phys. 121, 023107 (2017).

[165] M. Liao, W. Gao, T. Cheng, X. Xue, Z. Duan, D. Deng, H. Kawashima, T. Suzuki, and Y. Ohishi, 
Five-octave-spanning supercontinuum generation in fluoride glass, Appl. Phys. Express 6, 032503 (2013).

[166] M. Liao, W. Gao, T. Cheng, Z. Duan, X. Xue, H. Kawashima, T. Suzuki, and Y. Ohishi, Ultrabroad supercontinuum generation through filamentation in tellurite glass, Laser Phys. Lett. 10, 036002 (2013).

[167] P. Béjot, F. Billard, C. Peureux, T. Diard, J. Picot-Clémente, C. Strutynski, P. Mathey, O. Mouawad, O. Faucher, K. Nagasaka, Y. Ohishi, and F. Smektala, Filamentation-induced spectral broadening and pulse shortening of infrared pulses in Tellurite glass, Opt. Commun. 380, 245-249 (2016).

[168] Y. Yu, X. Gai, T. Wang, P. Ma, R. Wang, Z. Yang, D.-Y. Choi, S. Madden, and B. Luther-Davies, Mid-infrared supercontinuum generation in chalcogenides, Opt. Mater. Express 3, 1075-1086 (2013).

[169] O. Mouawad, P. Béjot, F. Billard, P. Mathey, B. Kibler, F. Désévédavy, G. Gadret, J.-C. Jules, O. Faucher, and F. Smektala, Mid-infrared filamentation-induced supercontinuum in As-S and an As-free $\mathrm{Ge}-\mathrm{S}$ counterpart chalcogenide glasses, Appl. Phys. B 121, 433-438 (2015).

[170] A.M. Stingel, H. Vanselous, and P.B. Petersen, Covering the vibrational spectrum with microjoule mid-infrared supercontinuum pulses in nonlinear optical applications, J. Opt. Soc. Am. B 34, 1163-1168 (2017).

[171] W.L. Smith, P. Liu, and N. Bloembergen, Superbroadening in $\mathrm{H}_{2} \mathrm{O}$ and $\mathrm{D}_{2} \mathrm{O}$ by self-focused picosecond pulses from a YAlG:Nd laser, Phys. Rev. A 15, 2396-2403 (1977).

[172] I. Golub, Optical characteristics of supercontinuum generation, Opt. Lett. 15, 305-307 (1990).

[173] G.S. He, G.C. Xu, Y. Cui, and P.N. Prasad, Difference of spectral superbroadening behavior in Kerr-type and non-Kerr-type liquids pumped with ultrashort laser pulses, Appl. Opt. 32, 45074512 (1993).

[174] A. Brodeur, F.A. Ilkov, and S.L. Chin, Beam filamentation and the white light continuum divergence, Opt. Commun. 129, 193-198 (1996).
[175] A. Dubietis, G. Tamošauskas, I. Diomin, and A. Varanavičius, Self-guided propagation of femtosecond light pulses in water, Opt. Lett. 28, 1269-1271 (2003).

[176] W. Liu, O. Kosareva, I.S. Golubtsov, A. Iwasaki, A. Becker, V.P. Kandidov, and S.L. Chin, Random deflection of the white light beam during selffocusing and filamentation of a femtosecond laser pulse in water, Appl. Phys. B 75, 595-599 (2002).

[177] W. Liu, O. Kosareva, I.S. Golubtsov, A. Iwasaki, A. Becker, V.P. Kandidov, and S.L. Chin, Femtosecond laser pulse filamentation versus optical breakdown in $\mathrm{H}_{2} \mathrm{O}$, Appl. Phys. B 76, 215 229 (2003).

[178] V.P. Kandidov, O.G. Kosareva, I.S. Golubtsov, W. Liu, A. Becker, N. Aközbek, C.M. Bowden, and S.L. Chin, Selftransformation of a powerful femtosecond laser pulse into a white-light laser pulse in bulk optical media (or supercontinuum generation), Appl. Phys. B 77, 149-165 (2003).

[179] J. Liu, H. Schroeder, S.L. Chin, R. Li, and $\mathrm{Z}$. Xu, Nonlinear propagation of fs laser pulses in liquids and evolution of supercontinuum generation, Opt. Express 13, 10248-10259 (2005).

[180] A.N. Tcypkin, S.E. Putilin, M.V. Melnik, E.A. Makarov, V.G. Bespalov, and S.A. Kozlov, Generation of high-intensity spectral supercontinuum of more than two octaves in a water jet, Appl. Opt. 55, 8390-8394 (2016).

[181] P. Vasa, J.A. Dharmadhikari, A.K. Dharmadhikari, R. Sharma, M. Singh, and D. Mathur, Supercontinuum generation in water by intense, femtosecond laser pulses under anomalous chromatic dispersion, Phys. Rev. A 89, 043834 (2014).

[182] J.A. Dharmadhikari, G. Steinmeyer, G. Gopakumar, D. Mathur, and A.K. Dharmadhikari, Femtosecond supercontinuum generation in water in the vicinity of absorption bands, Opt. Lett. 41, 3475-3478 (2016).

[183] M. Ziolek, R. Naskrecki, and J. Karolczak, Some temporal and spectral properties of femtosecond supercontinuum important in pumpprobe spectroscopy, Opt. Commun. 241, 221229 (2004). 
[184] V. Kartazaev and R.R. Alfano, Polarization properties of SC generated in $\mathrm{CaF}_{2}$, Opt. Commun. 281, 463-468 (2008).

[185] P.J.M. Johnson, V.I. Prokhorenko, and R.J.D. Miller, Stable UV to IR supercontinuum generation in calcium fluoride with conserved circular polarization states, Opt. Express 17, 21488-21496 (2009).

[186] P. Tzankov, I. Buchvarov, and T. Fiebig, Broadband optical parametric amplification in the near UV-VIS, Opt. Commun. 203, 107-113 (2002).

[187] I. Buchvarov, A. Trifonov, and T. Fiebig, Toward an understanding of white-light generation in cubic media - polarization properties across the entire spectral range, Opt. Lett. 32, 1539-1541 (2007).

[188] J. Zeller, J. Jaspara, W. Rudolph, and M. SheikBahae, Spectro-temporal characterization of a femtosecond white-light continuum by transient grating diffraction, Opt. Commun. 185, 133-137 (2000).

[189] N. Krebs, I. Pugliesi, J. Hauer, and E. Riedle, Two-dimensional Fourier transform spectroscopy in the ultraviolet with sub-20 fs pump pulses and $250-720 \mathrm{~nm}$ supercontinuum probe, New J. Phys. 15, 085016 (2013).

[190] R. Huber, H. Satzger, W.Zinth, and J. Wachtveitl, Noncollinear optical parametric amplifiers with output parameters improved by the applications of a white light continuum generated in $\mathrm{CaF}_{2}$, Opt. Commun. 194, 443-448 (2001).

[191] J. Kohl-Landgraf, J.-E. Nimsch, and J. Wachtveitl, $\mathrm{Li} F$, an underestimated supercontinuum source in femtosecond transient absorption spectroscopy, Opt. Express 21, 17060-17065 (2013).

[192] A.K. Dharmadhikari, F.A. Rajgara, N.C.S. Reddy, A.S. Sandhu, and D. Mathur, Highly efficient white light generation from barium fluoride, Opt. Express 12, 695-700 (2004).

[193] A.K. Dharmadhikari, K. Alti, J.A. Dharmadhikari, and D. Mathur, Control of the onset of filamentation in condensed media, Phys. Rev. A 76, 033811 (2007).

[194] A.K. Dharmadhikari, F.A. Rajgara, and D. Mathur, Plasma effects and the modulation of white light spectra in the propagation of ultrashort, high-power laser pulses in barium fluoride, Appl. Phys. B 82, 575-583 (2006).

[195] H. Liang, P. Krogen, R. Grynko, O. Novak, C.-L. Chang, G.J. Stein, D. Weerawarne, B. Shim, F.X. Kärtner, and K.-H. Hong, Three-octavespanningsupercontinuumgenerationandsub-twocycle self-compression of mid-infrared filaments in dielectrics, Opt. Lett. 40, 1069-1072 (2015).

[196] A.E. Dormidonov, V.O. Kompanets, S.V.Chekalin, and V.P. Kandidov, Giantically blue-shifted visible light in femtosecond mid-IR filament in fluorides, Opt. Express 23, 29202-29210 (2015).

[197] S.V. Chekalin, V.O. Kompanets, A.E. Dormidonov, E.D. Zaloznaya, and V.P. Kandidov, Supercontinuum spectrum upon filamentation of laser pulses under conditions of strong and weak anomalous group velocity dispersion in transparent dielectrics, Quantum Electron. 47, 252-258 (2017).

[198] A.E. Dormidonov, V.O. Kompanets, S.V. Chekalin, and V.P. Kandidov, Dispersion of the antistokes band in the spectrum of a light bullet of a femtosecond filament, JETP Lett. 104, 175-179 (2016).

[199] S.V. Chekalin, V.O. Kompanets, A.E. Dormidonov, and V.P. Kandidov, Influence of induced colour centres on the frequency - angular spectrum of a light bullet of mid-IR radiation in lithium fluoride, Quant. Electron. 47, 259-265 (2017).

[200] A. Marcinkevičiūtè, N. Garejev, R. Šuminas, G. Tamošauskas, and A. Dubietis, A compact, self-compression-based sub-3 optical cycle source in the 3-4 $\mu \mathrm{m}$ spectral range, J. Opt. 19, 105505 (2017).

[201] M.K. Reed, M.K. Steiner-Shepard, and D.K. Negus, Widely tunable femtosecond optical parametric amplifier at $250 \mathrm{kHz}$ with a Ti:sapphire regenerative amplifier, Opt. Lett. 19, 1855-1857 (1994).

[202] M.K. Reed, M.K. Steiner-Shepard, M.S. Armas, and D.K. Negus, Microjoule-energy ultrafast optical parametric amplifiers, J. Opt. Soc. Am. B 12, 2229-2236 (1995). 
[203] C. Manzoni and G. Cerullo, Design criteria for ultrafast optical parametric amplifiers, J. Opt. 18, 103501 (2016).

[204] M. Bradler and E. Riedle, Sub-20 fs $\mu$ J-energy pulses tunable down to the near-UV from a $1 \mathrm{MHz}$ Yb-fiber laser system, Opt. Lett. 39, 2588-2591 (2014).

[205] T. Imran and G. Figueira, Intensity-phase characterization of white-light continuum generated in sapphire by $280 \mathrm{fs}$ laser pulses at $1053 \mathrm{~nm}$, J. Opt. 14, 035201 (2012).

[206] R. Budriūnas, T. Stanislauskas, and A. Varanavičius, Passively CEP-stabilized frontend for few cycle terawatt OPCPA system, J. Opt. 17, 094008 (2015).

[207] H. Fattahi, H. Wang, A. Alismail, G. Arisholm, V. Pervak, A.M. Azzeer, and F. Krausz, Near-PHzbandwidth, phasestable continua generated from a Yb:YAG thin-disk amplifier, Opt. Express 24, 24337-24346 (2016).

[208] M. Bradler, P. Baum, and E. Riedle, Continuum generation in laser host materials towards table-top OPCPA, in: Proceedings of International Conference on Ultrafast Phenomena, paper ME25 (2010).

[209] M. Bradler and E. Riedle, Continuum generation in laser host materials with pump pulse durations covering the entire femtosecond regime, in: Advances in Optical Materials, OSA Technical Digest (CD), paper AMD4 (2011).

[210] A.-L. Calendron, H. Çankaya, G. Cirmi, and F.X. Kärtner, White-light generation with sub-ps pulses, Opt. Express 23, 13866-13879 (2015).

[211] I. Gražulevičiūtė, M. Skeivytė, E. Keblytė, J. Galinis, G. Tamošauskas, and A. Dubietis, Supercontinuum generation in YAG and sapphire with picosecond laser pulses, Lith. J. Phys. 55, 110-116 (2015).

[212] J. Galinis, G. Tamošauskas, I. Gražulevičiūte, E. Keblyte, V. Jukna, and A. Dubietis, Filamentation and supercontinuum generation in solid-state dielectric media with picosecond laser pulses, Phys. Rev. A 92, 033857 (2015).

[213] L. Kasmi, D. Kreier, M. Bradler, E. Riedle, and P. Baum, Femtosecond single-electron pulses generated by two-photon photoemission close to the work function, New J. Phys. 17, 033008 (2015).
[214] M. Emons, A. Steinmann, T. Binhammer, G. Palmer, M. Schultze, and U. Morgner, Sub-10fs pulses from a MHz-NOPA with pulse energies of $0.4 \mu \mathrm{J}$, Opt. Express 18, 1191-1196 (2010).

[215] R. Riedel, A. Stephanides, M.J. Prandolini, B. Gronloh, B. Jungbluth, T. Mans, and F. Tavella, Power scaling of supercontinuum seeded megahertz-repetition rate optical parametric chirped pulse amplifiers, Opt. Lett. 39, 1422-1424 (2014).

[216] N. Thiré, R. Maksimenka, B. Kiss, C. Ferchaud, P. Bizouard, E. Cormier, K. Osvay, and N. Forget, $4-\mathrm{W}, 100-\mathrm{kHz}$, few-cycle mid-infrared source with sub-100-mrad carrier-envelope phase noise, Opt. Express 25, 1505-1514 (2017).

[217] L. Indra, F. Batysta, P. Hř́bek, J. Novák, Z. Hubka, J.T. Green, R. Antipenkov, R. Boge, J.A. Naylon, P. Bakule, and B. Rus, Picosecond pulse generated supercontinuum as a stable seed for OPCPA, Opt. Lett. 42, 843-846 (2017).

[218] P. Rigaud, A. van de Walle, M. Hanna, N. Forget, F. Guichard, Y. Zaouter, K. Guesmi, F. Druon, and P. Georges, Supercontinuum-seeded few-cycle mid-infrared OPCPA system, Opt. Express 24, 26494-26502 (2016).

[219] G.M. Archipovaite, S. Petit, J.-C. Delagnes, and E. Cormier, $100 \mathrm{kHz} \mathrm{Yb}$-fiber laser pumped $3 \mu \mathrm{m}$ optical parametric amplifier for probing solidstate systems in the strong field regime, Opt. Lett. 42, 891-894 (2017).

[220] T. Kanai, P. Malevich, S.S. Kangaparambil, K. Ishida, M. Mizui, K. Yamanouchi, H. Hoogland, R. Holzwarth, A. Pugzlys, and A. Baltuska, Parametric amplification of 100 fs mid-infrared pulses in $\mathrm{ZnGeP}_{2}$ driven by a Ho:YAG chirpedpulse amplifier, Opt. Lett. 42, 683-686 (2017).

[221] P. Malevich, T. Kanai, H. Hoogland, R. Holzwarth, A. Baltuška, and A. Pugžlys, Broadband mid-infrared pulses from potassium titanyl arsenate/zinc germanium phosphate optical parametric amplifier pumped by Tm, Ho-fiber-seeded Ho:YAG chirped-pulse amplifier, Opt. Lett. 41, 930-933 (2017).

[222] W. Ryba-Romanowski, B. Macalik, A. Strzęp, R. Lisiecki, P. Solarz, and R.M. Kowalski, Spectral transformation of infrared ultrashort pulses in laser crystals, Opt. Mater. 36, 1745-1748 (2014). 
[223] R.S.S. Kumar, K.L.N. Deepak, and D.N. Rao, Control of the polarization properties of the supercontinuum generation in a noncentrosymmetric crystal, Opt. Lett. 33, 1198-1200 (2008).

[224] R.S.S. Kumar, K.L.N. Deepak, and D.N. Rao, Depolarization properties of the femtosecond supercontinuum generated in condensed media, Phys. Rev. A 78, 043818 (2008).

[225] J. Yu, H. Jiang, H. Yang, and Q. Gong, Depolarization of white light generated by femtosecond laser pulse in KDP crystals, J. Opt. Soc. Am. B 28, 1566-1570 (2011).

[226] J. Rolle, L. Bergé, G. Duchateau, and S. Skupin, Filamentation of ultrashort laser pulses in silica glass and KDP crystals: A comparative study, Phys. Rev. A 90, 023834 (2014).

[227] Y. Wang, H. Ni, W. Zhan, J. Yuan, and R. Wang, Supercontinuum and $\mathrm{THz}$ generation from $\mathrm{Ni}$ implanted $\mathrm{LiNbO}_{3}$ under $800 \mathrm{~nm}$ laser excitation, Opt. Commun. 291, 334-336 (2013).

[228] P. Vasa, K. Dota, M. Singh, D. Kushavah, B.P. Singh, and D. Mathur, Power- and polarization-dependent supercontinuum generation in $\alpha-\mathrm{BaB}_{2} \mathrm{O}_{4}$ crystals by intense, near-infrared, femtosecond laser pulses, Phys. Rev. A 91, 053837 (2015).

[229] N.K.M.N. Srinivas, S.S. Harsha, and D.N. Rao, Femtosecond supercontinuum generation in a quadratic nonlinear medium (KDP), Opt. Express 13, 3224-3229 (2005).

[230] R.S.S. Kumar, S.S. Harsha, and D.N. Rao, Broadband supercontinuum generation in a single potassium di-hydrogen phosphate (KDP) crystal achieved in tandem with sum frequency generation, Appl. Phys. B 86, 615-621 (2007).

[231] L. Wang, Y.X. Fan, H. Zhu, Z.D. Yan, H. Zeng, H.-T. Wang, S.N. Zhu, and Z.L. Wang, Broadband colored-crescent generation in a single $\beta$-bariumborate crystal by intense femtosecond pulses, Phys. Rev. A 84, 063831 (2011).

[232] S.A. Ali, P.B. Bisht, A. Nautiyal, V. Shukla, K.S. Bindra, and S.M. Oak, Conical emission in $\beta$-barium borate under femtosecond pumping with phase matching angles away from second harmonic generation, J. Opt. Soc. Am. B 27, 1751-1756 (2010).
[233] G.I. Stegeman, D.J. Hagan, and L. Torner, $\chi^{(2)}$ cascading phenomena and their applications to all-optical signal processing, mode-locking, pulse compression and solitons, Opt. Quant. Electron. 28, 1691-1740 (1996).

[234] M. Conforti, F. Baronio, and C. De Angelis, Modeling of ultrabroadband and single-cycle phenomena in anisotropic quadratic crystals, J. Opt. Soc. Am. B 28, 1231-1237 (2011).

[235] M. Conforti and F. Baronio, Extreme highintensity and ultrabroadband interactions in anisotropic $\beta-\mathrm{BaB}_{2} \mathrm{O}_{4}$ crystals, J. Opt. Soc. Am. B 30, 1041-1047 (2013).

[236] B.B. Zhou, A. Chong, F.W. Wise, and M. Bache, Ultrafast and octave-spanning optical nonlinearities from strongly phase-mismatched quadratic interactions, Phys. Rev. Lett. 109, 043902 (2012).

[237] M. Bache, H. Guo, and B. Zhou, Generating mid-IR octave-spanning supercontinua and fewcycle pulses with solitons in phase-mismatched quadratic nonlinear crystals, Opt. Mater. Express 3, 1647-1657 (2013).

[238] B. Zhou, H. Guo, and M. Bache, Energetic mid-IR femtosecond pulse generation by selfdefocusing soliton-induced dispersive waves in a bulk quadratic nonlinear crystal, Opt. Express. 23, 6924-6936 (2015).

[239] B. Zhou and M. Bache, Dispersive waves induced by self-defocusing temporal solitons in a beta-barium-borate crystal, Opt. Lett. 40, 42574260 (2015).

[240] B. Zhou and M. Bache, Multiple-octave spanning mid-IR supercontinuum generation in bulk quadratic nonlinear crystals, APL Photon. 1, 050802 (2016).

[241] K. Krupa, A. Labruyère, A. Tonello, B.M. Shalaby, V. Couderc, F. Baronio, and A.B. Aceves, Polychromatic filament in quadratic media: spatial and spectral shaping of light in crystals, Optica 2, 1058-1064 (2015).

[242] R. Šuminas, G. Tamošauskas, V. Jukna, A. Couairon, and A. Dubietis, Second-order cascading-assisted filamentation and controllable supercontinuum generation in birefringent crystals, Opt. Express 25, 6746-6756 (2017). 
[243] C. Vicario, B. Monoszlai, G. Arisholm, and C.P. Hauri, Generation of 1.5-octave intense infrared pulses by nonlinear interactions in DAST crystal, J. Opt. 17, 094005 (2015).

[244] A. Kessel, S.A. Trushin, N. Karpowicz, C. Skrobol, S. Klingebiel, C. Wandt, and S. Karsch, Generation of multi-octave spanning high-energy pulses by cascaded nonlinear processes in BBO, Opt. Express 24, 5628-5637 (2016).

[245] A.H. Chin, O.G. Calderón, and J. Kono, Extreme midinfrared nonlinear optics in semiconductors, Phys. Rev. Lett. 86, 3292-3295 (2001).

[246] P.B. Corkum, P.P. Ho, R.R. Alfano, and J.T. Manassah, Generation of infrared supercontinuum covering 3-14 $\mu \mathrm{m}$ in dielectrics and semiconductors, Opt. Lett. 10, 624-626 (1985).

[247] J.J. Pigeon, S.YA. Tochitsky, C. Gong, and C. Joshi, Supercontinuum generation from 2 to $20 \mu \mathrm{m}$ in GaAs pumped by picosecond $\mathrm{CO}_{2}$ laser pulses, Opt. Lett. 39, 3246-3249 (2014).

[248] S. Ashihara and Y. Kawahara, Spectral broadening of mid-infrared femtosecond pulses in GaAs, Opt. Lett. 34, 3839-3841 (2009).

[249] A.A. Lanin, A.A. Voronin, E.A. Stepanov, A.B. Fedotov, and A.M. Zheltikov, Frequencytunable sub-two-cycle 60-MW-peak-power freespace waveforms in the mid-infrared, Opt. Lett. 39, 6430-6433 (2014).

[250] A.A. Lanin, A.A. Voronin, E.A. Stepanov, A.B. Fedotov, and A.M. Zheltikov, Multioctave, 3-18 $\mu \mathrm{m}$ sub-two-cycle supercontinua from selfcompressing, self-focusing soliton transients in a solid, Opt. Lett. 40, 974-977 (2015).

[251] M. Durand, A. Houard, K. Lim, A. Durécu, O. Vasseur, and M. Richardson, Study of filamentation threshold in zinc selenide, Opt. Express 22, 5852-5858 (2014).

[252] O. Mouawad, P. Béjot, F. Billard, P. Mathey, B. Kibler, F. Désévédavy, G. Gadret, J.-C. Jules, O. Faucher, and F. Smektala, Filament-induced visible-to-mid-IR supercontinuum in a $\mathrm{ZnSe}$ crystal: Towards multi-octave supercontinuum absorption spectroscopy, Opt. Mater. 60, 355358 (2016).
[253] R. Šuminas, G. Tamošauskas, G. Valiulis, V. Jukna, A. Couairon, and A. Dubietis, Multioctave spanning nonlinear interactions induced by femtosecond filamentation in polycrystalline ZnSe, Appl. Phys. Lett. 110, 241106 (2017).

[254] M. Baudrier-Raybaut, R. Häıdar, Ph. Kupecek, $\mathrm{Ph}$. Lemasson, and E. Rosencher, Random quasiphase-matching in bulk polycrystalline isotropic nonlinear materials, Nature 432, 374-376 (2004).

[255] A.S. Sandhu, S. Banerjee, and D. Goswami, Suppression of supercontinuum generation with circularly polarized light, Opt. Commun. 181, 101-107 (2000).

[256] A. Srivastava and A. Goswami, Control of supercontinuum generation with polarization of incident laser pulses, Appl. Phys. B 77, 325-328 (2003).

[257] D. Schumacher, Controlling continuum generation, Opt. Lett. 27, 451-453 (2002).

[258] J.A. Dharmadhikari, A.K. Dharmadhikari, K. Dota, and D. Mathur, Influencing supercontinuum generation by phase distorting an ultrashort laser pulse, Opt. Lett. 40, 241-243 (2015).

[259] J.V. Thompson, P.A. Zhokhov, M.M. Springer, A.J. Traverso, V.V. Yakovlev, A.M. Zheltikov, A.V. Sokolov, and M.O. Scully, Amplitude concentration in a phase-modulated spectrum due to femtosecond filamentation, Sci. Rep. 7, 43367 (2017).

[260] V. Kartazaev and R.R. Alfano, Supercontinuum generated in calcite with chirped femtosecond pulses, Opt. Lett. 32, 3293-3295 (2007).

[261] K. Wang, L. Qian, H. Luo, P. Yuan, and H. Zhu, Ultrabroad supercontinuum generation by femtosecond dual-wavelength pumping in sapphire, Opt. Express 14, 6366-6371 (2006).

[262] A.A. Kolomenskii, J. Strohaber, N. Kaya, G. Kaya, A.V. Sokolov, and H.A. Schuessler, White-light generation control with crossing beams of femtosecond laser pulses, Opt. Express 24, 282-293 (2016).

[263] K. Stelmaszczyk, P. Rohwetter, Y. Petit, M. Fechner, J. Kasparian, J.-P. Wolf, and L. Wöste, White-light symmetrization by the interaction of multifilamenting beams, Phys. Rev. A 79, 053856 (2009). 
[264] C. Romero, R. Borrego-Varillas, A. Camino, G. Mínguez-Vega, O. Mendoza-Yero, J. Hernández-Toro, and J. R. Vázquez de Aldana, Diffractive optics for spectral control of the supercontinuum generated in sapphire with femtosecond pulses, Opt. Express 19, 4977-4984 (2011).

[265] R. Borrego-Varillas, C. Romero, O. MendozaYero, G. Mínguez-Vega, I. Gallardo, and J. R. Vázquez de Aldana, Femtosecond filamentation in sapphire with diffractive lenses, J. Opt. Soc. Am. B 30, 2059-2065 (2013).

[266] N. Kaya, J. Strohaber, A.A. Kolomenskii, G. Kaya, H. Schroeder, and H.A. Schuessler, White-light generation using spatially-structured beams of femtosecond radiation, Opt. Express 20, 13337-13346 (2012).

[267] R. Borrego-Varillas, J. Perez-Vizcaino, O. Mendoza-Yero, G. Minguez-Vega, J.R.V. de Aldana, and J. Lancis, Controlled multibeam supercontinuum generation with a spatial light modulator, IEEE Photon. Technol. Lett. 26, 1661-1664 (2014).

[268] Y. Zhong, H. Diao, Z. Zeng, Y. Zheng, X. Ge, $\mathrm{R}$. Li, and Z. Xu, CEP-controlled supercontinuum generation during filamentation with mid-infrared laser pulse, Opt. Express 22, 29170-29178 (2014).

[269] T. Jimbo, V.L. Caplan, Q.X. Li, Q.Z. Wang, P.P. Ho, and R.R. Alfano, Enhancement of ultrafast supercontinuum generation in water by the addition of $\mathrm{Zn}^{2+}$ and $\mathrm{K}^{+}$cations, Opt. Lett. 12, 477-479 (1977).

[270] C. Wang, Y. Fu, Z. Zhou, Y. Cheng, and Z. Xu, Femtosecond filamentation and supercontinuum generation in silver-nanoparticle-doped water, Appl. Phys. Lett. 90, 181119 (2007).

[271] P. Vasa, M. Singh, R. Bernard, A.K. Dharmadhikari, J.A. Dharmadhikari, and D. Mathur, Supercontinuum generation in water doped with gold nanoparticles, Appl. Phys. Lett. 103, 111109 (2013).

[272] R. Driben, A. Husakou, and J. Herrmann, Supercontinuum generation in aqueous colloids containing silver nanoparticles, Opt. Lett. 34, 2132-2134 (2009).
[273] Y.N. Kulchin, S.S. Golik, D.Y. Proschenko, A.A. Chekhlenok, I.V. Postnova, A.Y. Mayor, and Y.A. Shchipunov, Supercontinuum generation and filamentation of ultrashort laser pulses in hybrid silicate nanocomposite materials on the basis of polysaccharides and hyperbranched polyglycidols, Quant. Electron. 43, 370-373 (2013).

[274] L. Wang, Y.-X. Fan, Z.-D. Yan, H.-T. Wang, and Z.-L. Wang, Flat-plateau supercontinuum generation in liquid absorptive medium by femtosecond filamentation, Opt. Lett. 35, 2925-2927 (2010).

[275] C. Santhosh, A.K. Dharmadhikari, J.A. Dharmadhikari, K. Alti, and D. Mathur, Supercontinuum generation in macromolecular media, Appl. Phys. B 99, 427-432 (2010).

[276] D. Paipulas, A. Balskienè, and V. Sirutkaitis, Experimental study of filamentation and supercontinuum generation in laser-modified fused silica, Lith. J. Phys. 52, 327-333 (2012).

[277] C. Rolland and P.B. Corkum, Compression of high-power optical pulses, J. Opt. Soc. Am. B 5, 641-647 (1988).

[278] E. Mével, O. Tcherbakoff, F. Salin, and E. Constant, Extracavity compression technique for high-energy femtosecond pulses, J. Opt. Soc. Am. B 20, 105-108 (2003).

[279] C.-H. Lu, Y.-J. Tsou, H.-Y. Chen, B.-H. Chen, Y.-C. Cheng, S.-D. Yang, M.-C. Chen, C.-C. Hsu, and A. H. Kung, Generation of intense supercontinuum in condensed media, Optica 1, 400-406 (2014).

[280] Y.-C. Cheng, C.-H. Lu, Y.-Y. Lin, and A.H. Kung, Supercontinuum generation in a multi-plate medium, Opt. Express 24, 7224-7231 (2016).

[281] M. Seidel, G. Arisholm, J. Brons, V. Pervak, and O. Pronin, All solid-state spectral broadening: an average and peak power scalable method for compression of ultrashort pulses, Opt. Express 24, 9412-9428 (2016).

[282] P. He, Y. Liu, K. Zhao, H. Teng, X. He, P. Huang, H. Huang, S. Zhong, Y. Jiang, S. Fang, X. Hou, and Z. Wei, High-efficiency supercontinuum generation in solid thin plates at 0.1 TW level, Opt. Lett. 42, 474-477 (2017). 
[283] R. Budriūnas, D. Kučinskas, and A. Varanavičius, High-energy continuum generation in an array of thin plates pumped by tunable femtosecond IR pulses, Appl. Phys. B 123, 212 (2017).

[284] A.A. Voronin, A.M. Zheltikov, T. Ditmire, B. Rus, and G. Korn, Subexawatt few-cycle light wave generation via multipetawatt pulse compression, Opt. Commun. 291, 299-303 (2013).

[285] M. Hemmer, M. Baudisch, A. Thai, A. Couairon, and J. Biegert, Self-compression to sub3 -cycle duration of mid-infrared optical pulses in dielectrics, Opt. Express 21, 28095-28102 (2013).

[286] M. Baudisch, H. Pires, H. Ishizuki, T. Taira, M. Hemmer, and J. Biegert, Sub-4-optical-cycle, 340 MW peak power, high stability mid-IR source at $160 \mathrm{kHz}$, J. Opt. 17, 094002 (2015).

[287] B.G. Bravy, V.M. Gordienko, and V.T. Platonenko, Kerr effect-assisted self-compression in dielectric to single-cycle pulse width and to terawatt power level in mid-IR, Opt. Commun. 344, 7-11 (2015).

[288] B.G. Bravy, V.M. Gordienko, and V.T. Platonenko, Self-compression of terawatt level picosecond $10 \mu \mathrm{m}$ laser pulses in $\mathrm{NaCl}$, Laser Phys. Lett. 11, 065401 (2014).

[289] W. Li, Y. Li, Y. Xu, X. Guo, J. Lu, P. Wang, and Y. Leng, Design and simulation of a single-cycle source tunable from 2 to 10 micrometers, Opt. Express 25, 7101-7111 (2017).

[290] A.A. Voronin and A.M. Zheltikov, Asymptotically one-dimensional dynamics of high-peakpower ultrashort laser pulses, J. Opt. 18, 115501 (2016).

[291] A.A. Voronin and A.M. Zheltikov, Pulse selfcompression to single-cycle pulse widths a few decades above the selffocusing threshold, Phys. Rev. A 94, 023824 (2016).

[292] V. Shumakova, P. Malevich, S. Ališauskas, A. Voronin, A.M. Zheltikov, D. Faccio, D. Kartashov, A. Baltuška, and A. Pugžlys, Multi-millijoule few-cycle mid-infrared pulses through nonlinear self-compression in bulk, Nat. Commun. 7, 12877 (2016).

[293] N. Garejev, V. Jukna, G. Tamošauskas, M. Veličke, R. Šuminas, A. Couairon, and A. Dubietis, Odd harmonics-enhanced supercontinuum in bulk solid-state dielectric medium, Opt. Express 24, 17060-17068 (2016).

[294] E.A. Stepanov, A.A. Lanin, A.A. Voronin, A.B. Fedotov, and A.M. Zheltikov, Solid-state source of subcycle pulses in the midinfrared, Phys. Rev. Lett. 117, 043901 (2016).

[295] T.M. Kardaś, B. Ratajska-Gadomska, W. Gadomski, A. Lapini, and R. Righini, The role of stimulated Raman scattering in supercontinuum generation in bulk diamond, Opt. Express 21, 24201-24209 (2013).

[296] S.A. Frolov, V.I. Trunov, V.E. Leshchenko, and E.V. Pestryakov, Multi-octave supercontinuum generation with IR radiation filamentation in transparent solid-state media, Appl. Phys. B 122, 124 (2016).

[297] E. Mareev, V. Bagratashvili, N. Minaev, F. Potemkin, and V. Gordienko, Generation of an adjustable multi-octave supercontinuum under near-IR filamentation in gaseous, supercritical, and liquid carbon dioxide, Opt. Lett. 41, 57605763 (2016).

[298] A. Dubietis, P. Polesana, G. Valiulis, A. Stabinis, P. Di Trapani, and A. Piskarskas, Axial emission and spectral broadening in self-focusing of femtosecond Bessel beams, Opt. Express 15, 4168-4175 (2007).

[299] X. Sun, H. Gao, B. Zeng, S. Xu, W. Liu, Y. Cheng, Z. Xu, and G. Mu, Multiple filamentation generated by focusing femtosecond laser with axicon, Opt. Lett. 37, 857-859 (2012).

[300] K. Dota, A. Pathak, J.A. Dharmadhikari, D. Mathur, and A.K. Dharmadhikari, Femtosecond laser filamentation in condensed media with Bessel beams, Phys. Rev. A 86, 023808 (2012).

[301] K. Dota, J.A. Dharmadhikari, D. Mathur, and A.K. Dharmadhikari, Supercontinuum generation in barium fluoride using Bessel beams, Chin. J. Phys. 52, 431-439 (2014).

[302] P. Polynkin, M. Kolesik, and J. Moloney, Filamentation of femtosecond laser Airy beams in water, Phys. Rev. Lett. 103, 123902 (2009).

[303] C. Ament, M. Kolesik, J.V. Moloney, and P. Polynkin, Self-focusing dynamics of ultraintense accelerating Airy waveforms in water, Phys. Rev. A 86, 043842 (2012). 
[304] C. Gong, Z. Li, L.Q. Hua, W. Quan, and X.J. Liu, Angle-resolved conical emission spectra from filamentation in a solid with an Airy pattern and a Gaussian laser beam, Opt. Lett. 41, 43054308 (2016).

[305] D.N. Neshev, A. Dreischuh, G. Maleshkov, M. Samoc, and Y.S. Kivshar, Supercontinuum generation with optical vortices, Opt. Express 18, 18368-18373 (2010).
[306] G. Maleshkov, D.N. Neshev, E. Petrova, and A. Dreischuh, Filamentation and supercontinuum generation by singular beams in self-focusing nonlinear media, J. Opt. 13, 064015 (2011).

[307] H. Fattahi, H.G. Barros, M. Gorjan, T. Nubbemeyer, B. Alsaif, C.Y. Teisset, M. Schultze, S. Prinz, M. Haefner, M. Ueffing, et al., Thirdgeneration femtosecond technology, Optica 1, 45-63 (2014).

\title{
FEMTOSEKUNDINIO SUPERKONTINUUMO GENERAVIMAS PLAČIOS APERTŪROS KONDENSUOTOSE TERPĖSE
}

\author{
Apžvalga \\ A. Dubietis ${ }^{a}$, G. Tamošauskas ${ }^{a}$, R. Šuminas ${ }^{a}$, V. Jukna ${ }^{\text {b, c }}$, A. Couairon ${ }^{c}$ \\ a Vilniaus universiteto Lazeriniu tyrimu centras, Vilnius, Lietuva \\ ${ }^{\mathrm{b}}$ Taikomosios optikos laboratorija, Paryžiaus Saklè universitetas, Paleso, Prancūzija \\ ' Teorinès fizikos centras, Paryžiaus Saklè universitetas, Paleso, Prancūzija
}

\begin{abstract}
Santrauka
Netiesinis intensyvių femtosekundinių lazerio impulsų sklidimas plačios apertūros dielektrinèse terpèse lemia specifinių darinių - femtosekundinių šviesos gijų - susidarymą ir turi ịtakos didelio masto spektro plètrai, vadinamajai superkontinuumo generacijai. Femtosekundinio superkontinuumo generacija skaidriose kietakūnèse terpèse yra kompaktiškas, efektyvus bei derinimui nejautrus metodas generuoti koherentinę plataus spektro spinduliuotę bet kurioje optinio diapazono dalyje ir turintis daugybę taikymo galimybiu įvairiose šiuolaikinio mokslo, tiriančio ultrasparčiuosius vyksmus, srityse. Pastaruoju metu ši mokslinių tyrimų sritis pasiekė aukštą lygị tiek fizikinių procesų supratimo, tiek praktinių pasiekimų požiūriu. Straips-
\end{abstract}

nyje apžvelgiami femtosekundinio superkontinuumo generacijos rezultatai ịvairiose skaidriose kietakūnèse terpèse (nuo plačios draustinès juostos dielektriku iki puslaidininkių) ir ivvairiose optinio spektro srityse (nuo ultravioletinio iki viduriniojo infraraudonojo spektrinio diapazono). Akcentuojami naujausi eksperimentiniai pasiekimai, apimantys daugiaoktavio superkontinuumo generavimą žadinant femtosekundiniais impulsais vidurineje infraraudonojoje spektro srityje, spektro valdymą, plataus spektro spinduliuotes galios ir energijos didinimą bei paprastų, lanksčių ir patikimų impulsų spūdos metodų plètojimą, kurie leidžia generuoti keleto optinių ciklu trukmès impulsus ir gali būti lengvai integruojami $\mathfrak{i}$ daugeli šiuolaikinių ultrasparčiųjų lazerinių sistemų. 